\title{
On Seven-Brane Dependent Instanton Prefactors in F-theory
}

\author{
Mirjam Cvetič $^{1,2}$, Ron Donagi ${ }^{3}$, James Halverson ${ }^{1,4}$, and Joseph Marsano ${ }^{5,6}$ \\ ${ }^{1}$ Department of Physics and Astronomy, University of Pennsylvania \\ 209 S. 33rd St, Philadelphia, PA 19104 USA \\ ${ }^{2}$ Center for Applied Mathematics and Theoretical Physics \\ University of Maribor, Maribor, Slovenia \\ ${ }^{3}$ Department of Mathematics, University of Pennsylvania \\ 209 S. 33rd St, Philadelphia, PA 19104-6395 USA \\ ${ }^{4}$ Kavli Institute for Theoretical Physics, University of California \\ Santa Barbara, CA 93106-4030 USA \\ ${ }^{5}$ Enrico Fermi Institute, University of Chicago \\ 5640 S Ellis Ave, Chicago, IL 60637, USA \\ ${ }^{6}$ Sunrise Futures LLC \\ 30 S Wacker Suite 1706, Chicago, IL 60606 \\ cvetic@cvetic.hep.upenn.edu, donagi@math.upenn.edu, \\ jim@kitp.ucsb.edu, marsano@theory.caltech.edu
}

\begin{abstract}
We study the moduli-dependent prefactor of M5-instanton corrections to the superpotential in four-dimensional F-theory compactifications. In light of the M-theory and type IIb limits and also heterotic duality, we propose that the explicit moduli dependence of the prefactor can be computed by a study of zero modes localized at intersections between the instanton and seven-branes. We present an instanton prefactor in an $E_{6}$ F-theory GUT which does not admit a heterotic dual and show that it vanishes if and only if a point of $E_{8}$ enhancement is present in the instanton worldvolume. More generically, we discuss the relationship between points of $E_{8}$ and superpotential zeroes and give sufficient conditions for such a point to cause a zero, even for an $S U(5)$ GUT. We scan a large class of compactifications for instanton physics and demonstrate that many instantons have the same prefactor structure. We discuss the associated implications and complications for moduli stabilization. We present an explicit resolution and construction of G-flux in a generic $E_{6}$ GUT and identify a global compactification of the local model spectral cover which happens to facilitate prefactor computations. Via a Leray spectral sequence, we demonstrate the relationship between right-movers of heterotic worldsheet instantons, 3-3 strings of euclidean D3 instantons, and the Fermi zero modes of M5-instantons.
\end{abstract}




\section{Contents}

1 Introduction 3

1.1 Summary of Results and Paper Layout . . . . . . . . . . . . . . . 6

1.2 Notational Conventions . . . . . . . . . . . . . . . . . . 10

2 Heterotic Worldsheet Instantons 12

2.1 Fermi Zero Modes that Don't Couple to $V$. . . . . . . . . . . . . 13

2.2 Fermi Zero Modes that Couple to $V$ : the Pfaffian . . . . . . . . . . . . . 14

2.3 Moduli Dependence of the Pfaffian . . . . . . . . . . . . . . 17

3 F-theory and M5 Instantons $\quad 18$

$3.1 \quad$ F-theory Preliminaries . . . . . . . . . . . . . . . . 18

3.1.1 A Comment on the Compact Realization of $\mathcal{C}_{l o c}$ as $\left.\mathcal{C}_{F}\right|_{\pi^{*} B_{2}} \ldots .21$

3.2 M5 Instantons . . . . . . . . . . . . . . . . . . 23

3.2 .1 Fermi Zero Modes . . . . . . . . . . . . . . . . 23

3.2 .2 Chiral 2 -form . . . . . . . . . . . . . . 24

4 Heterotic/F-theory Duality 26

4.1 Heterotic/F-theory Duality . . . . . . . . . . . . . . . . 26

4.2 Heterotic WS Instantons and their M5 Counterparts . . . . . . . . . . . 30

4.3 Heterotic Right-Movers and M5 Fermi Zero Modes . . . . . . . . . . . 33

4.4 Heterotic Pfaffian and the Chiral 2-form Partition Function . . . . . . . 34

5 Instanton Prefactors in F-theory 36

5.1 Intuition and a Proposal: What to Compute . . . . . . . . . . . 37

5.1.1 Mathematical Relationship to the M5-brane Theory . . . . . . 39

5.2 Setting Up the Computation . . . . . . . . . . . . . . . 40

6 A Prefactor Scan in the Kreuzer-Skarke Database 42

7 Points of $E_{8}$ and Superpotential Zeroes 44

8 Illustrative Examples 48

8.1 Example One . . . . . . . . . . . . . . . . . . . . . 49

8.1.1 Flat bundles, Intermediate Jacobians, and the Prefactor . . . . . 51

8.2 Example Two: Prefactors without a heterotic dual . . . . . . . . . 52

8.2.1 Points of $E_{8}$ and the Vanishing of the Prefactor . . . . . . . . 55

8.3 Example Three: A New Prefactor . . . . . . . . . . . . . . 56

A Calabi-Yau Resolutions and the Spectral Divisor 5

A.1 Resolving 4-folds with a surface of $E_{6}$ singularities . . . . . . . . . . 59

A.2 Spectral Divisor, Heterotic Spectral Cover, and Higgs Bundle Spectral Cover ........................ 66

B Details of the Base in Example Two 78 


\section{Introduction}

The importance of instanton corrections in string compactifications was already recognized in the early days of the heterotic string [1-3, when it was found that hierarchical Yukawa couplings in $E_{6}$ grand unified theories can be generated by non-perturbative worldsheet instanton corrections to the superpotential. In type II theories, euclidean D-brane instanton corrections to the superpotential play a crucial role in the stabilization of moduli [4, 5] and can generate charged matter couplings which are forbidden in string perturbation theory by anomalous $U(1)$ symmetries [6 8 . Historically, it has often been the case that an understanding of instanton effects in one corner of the landscape sheds light on instanton effects in another via duality or a special limit. This includes, for example, the rederivation of heterotic worldsheet instanton corrections via duality with euclidean D1-instanton corrections [9] and an understanding of euclidean D3-instantons in four-dimensional compactifications of F-theory via three-dimensional compactifications of M-theory [10].

Over the last four years there has been renewed interest in $\mathcal{N}=1, d=4$ compactifications of F-theory, initiated by the possibility of obtaining semi-realistic grand unified theories [11,12]. This approach to GUT model building uniquely combines two advantages of the heterotic and type II superstrings, giving rise to interesting GUT physics through the presence of exceptional gauge structures while localizing the gauge degrees of freedom in the internal space. In addition to many works on local model-building, there have been many attempts to construct global models [13 28, and properly understand chirality inducing $G_{4}$-flux [29,39] and abelian symmetries [30,40,43]. A proper understanding of chirality inducing flux and abelian symmetries is crucial for the understanding of instanton physics in the weakly coupled type IIb limit, and given the recent progress on these issues in F-theory one would hope to understand F-theoretic instantons in this light.

In this work we study M5-instantons 1 in F-theory, focusing on the dependence of the instanton prefactor on seven-brane moduli. Lacking a fundamental definition of F-theory, any understanding of F-theoretic physics must be obtained by comparison to the M-theory limit, and also to a heterotic dual or the IIb limit when they exist. This applies to the study of instanton corrections, and to the authors' knowledge all works on the subject have utilized this perspective. This is particularly evident in the early work on the subject. For example, in [9] the well known arithmetic genus constraint $\chi\left(D, \mathcal{O}_{D}\right)=1$ on divisors wrapped by instantons which correct the superpotential was derived via an understanding of M5-instantons in $d=3$ compactifications of M-theory. It was found in 44] that in a very special Calabi-Yau fourfold an infinite number of divisors satisfying the arithmetic genus constraint exist, giving rise to an infinite number of superpotential corrections which exhibit an $E_{8}$ symmetry2. It also is known [45, 46, that M5-instantons in $d=3$ M-theory play a crucial role in recovering the physics of $d=4$ supersymmetric QCD in the F-theory limit. Via the M-theory limit, it was shown in [47] that superpotential zeroes occur when the moduli of spacetime filling D3-branes

\footnotetext{
${ }^{1}$ We choose to call these M5-instantons, since their behavior is most fundamentally captured by M5instantons in the defining $d=3 \mathrm{M}$-theory compactification. The instantons in F-theory are actually euclidean D3's, and the relationship to M5-instantons can be made concrete in the geometry.

${ }^{2}$ This is not to be confused with the points of $E_{8}$ enhancement discussed in this work.
} 
are tuned such that the D3-brane comes into contact with the instanton, due to the appearance of additional zero modes. The appearance of zero modes at codimension one subloci in moduli space will be the salient feature determining instanton physics in this paper, where the modes of relevance are essentially ED3-7 zero modes from the type IIb point of view.

Since the appearance of [11,12], there have been many works studying instantons in F-theory. In [48] it was shown how fermionic instanton zero modes in type IIb uplift to F-theory and the relationship of these modes to the arithmetic genus. Spectral cover techniques were used to describe instanton intersections with D7-branes. In [21] an instanton in a semi-realistic type IIb GUT was uplifted to F-theory and the cohomology determining the arithmetic genus was studied. Instantons in F-theory were discussed via dualities and limits in [49] and it was shown that the worldvolume theory of an M5-brane instanton reduces to an MSW CFT [50] if it is a particular surface fibration over a curve. In [51] it was shown that instanton flux in F-theory can alleviate the tension [52] which can occur between chiral matter instanton prefactors and moduli stabilization in the type IIb limit. In [30] $G_{4}$-flux and abelian symmetries were discussed in the spectral divisor formalism and some associated aspects of instanton physics were addressed. In [53] instanton zero modes in F-theory were studied via anomaly inflow and $(p, q)$-string junctions, and (in examples) some physics of the moduli dependent Pfaffian prefactor were understood via heterotic duality. It is known that worldvolume fluxes on the instanton can lift fermionic zero modes which would prevent the instanton from contributing to the superpotential [54]. In [55] it was shown that comparison to the type IIb limit can determine which theta divisor on the intermediate Jacobian corresponds to the prefactor of an M5-instanton correction.

In this paper we will study the computation and physical understanding of the moduli-dependent instanton prefactor via dualities and limits of F-theory. We will cast the discussion of instanton prefactors into the language of the M-theory and heterotic duals [56], and also the weakly coupled type IIb limit. We will not address the dependence of the instanton prefactor on spacetime filling D3-branes [47,57], but instead will focus solely on the seven-brane moduli dependence. While it is important to consider any moduli dependence in a string compactification, we find the seven-brane moduli dependence to be particularly interesting since it gives a connection between the effective potential on moduli space and four-dimensional gauge theories.

Let us briefly review the picture of ED3-instantons in type IIb, since much of the intuition is expected to carry over to F-theory. Consider a euclidean D3-brane instanton wrapping a divisor $D$ with associated Kähler modulus $T$ in a type IIb orientifold compactification on a Calabi-Yau threefold. Under certain circumstances, the ED3 can correct the superpotential, and the structure of the correction depends heavily on the fermionic instanton zero modes. At a given point in the D7-brane moduli space of a type IIb compactification, the coupling of fermionic ED3-D7 zero modes to matter fields charged under the four-dimensional gauge group can generate superpotential corrections with prefactors that contain the charged fields. The correction takes the schematic form [6] 8 ]

$$
\Delta W \sim \prod_{i} \Phi_{i}^{k_{i}} e^{-T}
$$

where the $\Phi_{i}$ are chiral supermultiplets charged under $G_{4 d}$. Such effects are crucial 
for phenomenological considerations, since instantons can generate leading order contributions to couplings which are forbidden in perturbation theory by anomalous $U(1)$ symmetries. For example, at weak coupling the $10_{M} 10_{M} 5_{H}$ of an $S U(5)$ GUT and Majorana right-handed neutrino mass terms are always forbidden, but can be generated by instantons [6, 7, 58. While corrections with matter prefactors are very interesting, we will not study them here.

In the absence of these ED3-D7 zero modes, the prefactor is a constant determined by the vacuum expectation values of the relevant moduli, including those determining the structure of seven-branes. The superpotential correction takes the schematic form

$$
\Delta W \sim A e^{-T},
$$

and in this paper we will study the prefactor ${ }^{3} A$ as a function of seven-brane moduli, denoted collectively as $\phi$. The functional form of $A(\phi)$ is determined by the spectrum of zero modes, which can change upon movement in moduli space as additional modes become massless. This can be studied concretely via techniques in algebraic geometry, where the modes are counted by bundle cohomology and the corresponding Hodge numbers can jump at subloci in moduli space. It goes without saying that understanding this structure is important for the physics of four-dimensional string compactifications.

We will be interested in the study of $A(\phi)$ for M5-instantons in F-theory. Qualitatively, there are two issues which we find particularly interesting, and we will focus on those. The first is to identify the physics which determines $A$ in a class of GUT compactifications, and in the process to give a method for the explicit computation of $A$ as a function of seven-brane moduli, which are geometrized in F-theory as complex structure moduli. We will see that the relevant zero modes are counted by the cohomology of a line bundle $\mathcal{L}_{A}$ on a distinguished spectral curve $c_{l o c}$. From a type IIb perspective, these zero modes can be thought of as ED3-D7 strings localized at curves where the instanton intersects the GUT seven-brane. Then the 7-brane dependent prefactor $A(\phi)$ has a zero whenever $\mathcal{L}_{A}$ obtains a section. A non-trivial polynomial $A(\phi)$ corresponds to the situation where $\mathcal{L}_{A}$ does not have a section for generic moduli, but obtains a section in codimension one, namely on the locus $A(\phi)=0$. We will see that this prescription matches nicely with expectations from heterotic duality and the type IIb limit, and we will also discuss the relationship to the M5-brane worldvolume theory.

The second issue we would like to discuss regards the structure of $A$. In all known examples, the polynomial $A$ takes the schematic form

$$
A=\prod_{i} f_{i}^{k_{i}}
$$

for some other polynomials $f_{i}$ and integers $k_{i}$, so that $A=0$ is a reducible codimension one subvariety in moduli space. We would like to study its components defined by $f_{i}=0$ and address whether these loci correspond to interesting seven-brane physics. Such a correspondence should not be surprising, since zeroes of $A$ occur when extra modes become light, which often occurs at points of singularity enhancement in seven-brane

\footnotetext{
${ }^{3}$ In type IIb and F-theory, the prefactor is in fact the Pfaffian of a chiral Dirac operator representing one loop fluctuations around the instanton background, but we will utilize an equivalent algebraic description [59] which seems to be more tractable.
} 
moduli space. One aspect we will study is the connection between superpotential zeroes and points of $E_{8}$ enhancement 4 , which are of phenomenological interest 61. It would also be interesting to address the powers $k_{i}$, though we will only say a few words about this here. Some of these issues have been discussed in the dual heterotic picture 62 64 and our thoughts on the associated physics in F-theory have been influenced by those works. However, here we are primarily interested in F-theory physics and will take the seven-brane perspective.

The moduli dependence of the instanton prefactor $A$ enters the effective potential on moduli space, and it would be interesting to study issues of moduli stabilization taking this dependence into account. For any given modulus $\phi$, it has been argued that the contributions to $V(\phi)$ from $A$ are subleading compared to contributions from the flux superpotential. However, for a given set of fluxes it is not guaranteed that the flux superpotential contributes to $V(\phi)$, and therefore contributions to the potential from $A$ may be relevant. For these contributions, it is worth noting that known examples have $k_{i} \geq 2$, so that $f_{i}=0$ loci are often critical points of the correction, and perhaps of the full effective potential.

\subsection{Summary of Results and Paper Layout}

Since this work builds on many others, let us review the contributions which the authors believe are new and put them into their proper context. We will first focus on new insights of this work, and then turn to illustrative examples and new technical progress.

\section{Conceptual Insights}

The primary result of this paper is a conjecture for the computation of M5-instanton prefactors as a function of complex structure moduli in four-dimensional F-theory compactifications, analogous to the heterotic work of [59], but not requiring a heterotic duat5. The complex structure moduli dependence determines seven brane structure and therefore has interesting implications for four dimensional gauge theories. Our result is applicable to four dimensional grand unified theories where the Higgsing of an $E_{8}$ gauge theory on the GUT seven-brane can be described by an $S U(n)$ spectral cover. This includes, for example, the relevant cases $G_{4 d}=E_{6}, S O(10)$, and $S U(5)$, and is the context for the work on global and local F-theory GUTs over the last four years. For simplicity we will sometimes specify to the $E_{6}$ case. Our result will utilize a spectral curve $c_{l o c}$ which is an $n$-sheeted cover of a curve $\Sigma \subset B_{3}$ where the instanton divisor intersects the GUT stack. Since $S U(n)$ spectral covers describe the Higgsing of a single local non-abelian gauge theory, our result likely has to be modified if another non-abelian singularity intersects the GUT stack. However, in such a case we still expect bundle cohomology on $\Sigma$ to play an important role, though it may not admit a spectral curve description. In this work we will not study $U(1)$ symmetries, though they likely play an interesting role in instanton physics when they exist. The moduli-dependence of the

\footnotetext{
${ }^{4}$ It is known 60] that one must be careful in the application of Kodaira's codimension one classification of singularities to codimension three singularities. The " $E_{8}$ " points here correspond to specific tunings of moduli VEVs, rather than the appearance of an extended $E_{8}$ Dynkin diagram after resolution of the codimension three singularity.

${ }^{5}$ Some aspects of instantons in compactifications without a heterotic dual were also discussed in [49].
} 
prefactor which we study is governed by the presence or absence of zero modes localized on $\Sigma$. They can be counted by studying moduli-dependent vector bundle cohomology on $\Sigma$. Alternatively, which will be our method of choice, the zero modes are counted by line bundle cohomology on $c_{l o c}$. In this case, the seven-brane moduli dependence of the prefactor emerges through the moduli in the defining equation of $c_{l o c}$. The cohomology which determines the prefactor is intuitive from the heterotic and type IIb perspectives. From the type IIb perspective, the localized modes are naturally interpreted as ED37 string zero modes. When a heterotic dual exists, on the other hand, the prefactor is known to be determined by line bundle cohomology on another curve $c_{\text {Het }}$. As we demonstrate, $c_{H e t}$ and $c_{l o c}$ are isomorphic whenever a heterotic dual exists. This allows us to understand more directly how line bundle cohomology on $c_{l o c}$ determines the partition function of the chiral 2-form on the M5 worldvolume through simple application of the cylinder map, which provides a map from the Jacobian $\mathcal{J}\left(c_{l o c}\right)$ to the intermediate Jacobian $\mathcal{J}(M 5)$. Modulo some issues related to the familiar half-integral shift, this maps the Riemann $\theta$ divisor of $\mathcal{J}_{c_{l o c}}$ to a $\theta$ divisor of $\mathcal{J}(M 5)$ that determines the 2 -form partition function. In the absence of a heterotic dual we still have the curve $c_{l o c}$ but we no longer have the cylinder map. Nevertheless, we believe that (part of) the M5 partition function continues to be related to cohomology on $c_{l o c}$ in a way that we make precise with spectral divisors. While we do not have a proof of this conjecture, we find it mathematically plausible and physically very well-motivated, particularly from the heterotic and type IIb perspectives. In the $S O(10)$ and $S U(5)$ case, it would also be important to study modes counted by cohomology of the associated bundles $\wedge^{k} V$ on $\Sigma$. We leave this for future work.

Since instanton corrections to the superpotential are important for moduli stabilization, we systematically study prefactors across a large class of F-theory compactifications. We perform a scan of all toric threefolds related to fine triangulations of three-dimensional reflexive polytopes (that is, the entire $d=3$ Kreuzer-Skarke list [65]) and study each as the base $B_{3}$ of an F-theory compactification with $G_{4 d}=E_{6}$. In each case, we study instantons subject to certain criteria, keeping track of the topological quantities which are sufficient to determine the instanton prefactor and tabulating the results. Interestingly, we find that there are $\mathcal{O}\left(10^{5}\right)$ instanton corrections in these compactifications, yet only $\mathcal{O}(10)$ distinct prefactor structures. Of these possible prefactors, only three have been explicitly computed, but these three make up over half of the prefactors in our scan. Two of the prefactors are identically zero, a very common possibility with clear (and difficult) implications for Kähler moduli stabilization. The other vanishes if and only if there exists a point of $E_{8}$ enhancement in the instanton worldvolume 6 . It would be interesting, though very computationally intensive, to compute the remaining prefactors. It would also be interesting to broaden the scan by looking at different flux parameters, four-dimensional gauge groups, or base manifolds.

It is interesting that points of $E_{8}$ enhancement seem to play a special role in instanton corrections, since they are known to be of phenomenological relevance [61, and we explore this relationship in an example-independent way. Note that the arguments we present can be made in both the heterotic computation on $c_{H e t}$ or the F-theory computation on $c_{l o c}$. Building on related arguments in the heterotic case [63, we write down

\footnotetext{
${ }^{6}$ By this we mean that there exists a singular fiber, naively [60] of $E_{8}$ type, above a point in the instanton worldvolume.
} 
sufficient conditions under which a point of $E_{8}$ enhancement in the instanton worldvolume will cause a prefactor zero. Such conditions are useful because they allow one to study prefactor zeroes caused by points of $E_{8}$ without having to explicitly perform the tedious prefactor computation. The connection between $E_{8}$ points and superpotential zeroes may not be surprising, since enhanced symmetry points in moduli space often exhibit additional light modes, which could cause the zero. While this is true and evident in examples, the arguments we present proving the conditions related to $E_{8}$ do not apply to points of $E_{7}$ or $E_{6}$, for example. Thus $E_{8}$ points seem to be special. It would be interesting to explore these ideas further.

\section{Illustrative Examples}

We discuss three illustrative examples, emphasizing the computation of the prefactor via the study of line bundle cohomology on $c_{l o c}$ and also studying the factorization and substructure of the prefactor. All examples have $G_{4 d}=E_{6}$. In the first example, we study the instanton prefactor in an F-theory compactification with a heterotic dual. The prefactor takes the form $A \sim f_{\Lambda}^{4}$, where $f_{\Lambda}$ is a polynomial which obtains a zero if and only if a particular degree 0 bundle $\Lambda$ obtains a section. The order of vanishing of $f_{\Lambda}$, 4 , is also the number of zero modes which become massless on the $f_{\Lambda}=0$ locus. This example was studied from an F-theory perspective in [53], but here we have understood aspects of the entire vanishing locus, as opposed to subloci within the $A=0$ locus. That a related bundle controls a polynomial factor in $A$ is the notion of 'reduction' 62. from the heterotic instanton literature. In the second example, we study an instanton prefactor in an F-theory geometry which does not admit a heterotic dual. There we have $A \sim f_{E 8}^{4}$, where $f_{E 8}=0$ if and only if there exists a point of $E_{8}$ enhancement in the instanton worldvolume. In the third example we utilize the same base as in the second example, but change the location of the GUT stack $B_{2}$. We perform a new prefactor computation and find that the prefactor is identically zero for all relevant moduli. These examples demonstrate how the instanton prefactor can be computed in terms of intrinsically F-theoretic data, even when a heterotic dual does not exist.

\section{Technical Progress}

One point of technical progress significantly aids the computation. Let us discuss it in generality before discussing the relevance for instanton computations. Consider an $E_{8}$ gauge theory on a seven-brane wrapped on a four-cycle $B_{2}$ in the F-theory base $B_{3}$. The Higgs bundle spectral cover is a spectral pair $\left(\mathcal{C}_{l o c}, \mathcal{N}_{\text {loc }}\right)$ which determines the Higgsing of the $E_{8}$ gauge theory to the GUT group. $\mathcal{C}_{l o c}$ is an $n$-sheeted cover of $B_{2}$ describing the $S U(n)$ bundle that Higgses $E_{8}$ and $\mathcal{N}_{l o c}$ is a line bundle on it. In previous work, a non-compact $\mathcal{C}_{l o c}$ was utilized to study F-theory GUTs, but here we demonstrate a natural way to realize a compact $\mathcal{C}_{l o c}$. In short, $\mathcal{C}_{l o c}$ is a surface in the Calabi-Yau fourfold which can be realized as a complete intersection of two divisors in the fourth exceptional divisor $E_{4}$ of the resolved ambient space. $E_{4}$ is an $\mathbb{F}_{1}$-fibration over $B_{2}$ which contains an ambient elliptic $C Y_{3} Z_{3, F}$ which contains $\mathcal{C}_{\text {loc }}$ as a divisor. When a heterotic dual exists, $Z_{3, F}$ is isomorphic to the heterotic $C Y_{3} Z_{H e t}$ and $\mathcal{C}_{\text {loc }}$ is isomorphic to the heterotic spectral cover $\mathcal{C}_{H e t}$. That the fourth exceptional divisor $E_{4}$ plays a special role in obtaining a nice compactification of an $S U(n)$ spectral cover is not surprising: if it sat in the third exceptional divisor $E_{3}$, then an $S U(4)$ gauge 
symmetry would admit a spectral cover description, which it does not. If, on the other hand, $\mathcal{C}_{l o c}$ sat in the fifth exceptional divisor $E_{5}$, this compactification of the spectral cover would describe cases of $S O(10)$ gauge symmetry, but not $S U(5)$. Simply put, this compactification of the spectral cover begins to exist when the spectral cover description of the gauge theory begins to exist.

Let us discuss why this is useful for instanton computations. With this nice compactification of $\mathcal{C}_{l o c}$ as a divisor in an ambient elliptic Calabi-Yau threefold, we can intersect with the instanton divisor to obtain the spectral curve of interest, $c_{l o c}$, as a divisor in an elliptic surface $\mathcal{E}$. Then the line bundle cohomology on $c_{l o c}$ which determines the prefactor can be studied using a Koszul sequence from line bundles on $\mathcal{E}$. This method is mathematically identical to that of [59], even though computing cohomology on $c_{l o c}$ in this way does not require the existence of a heterotic dual. In fact, given an F-theory base $B_{3}$ and two divisors wrapped by the GUT stack and instanton, it is trivial to compute the topological quantities which determine the structure of $c_{l o c}$ and the line bundle $\mathcal{L}_{A}$, the cohomology of which must be computed to determine the prefactor. This allows for an efficient systematic scan, as discussed.

In the end, we arrive at a prescription for computing (part of) the moduli dependence of the instanton prefactor in a straightforward way using well-established techniques. From a practical point of view in a given example, studying line bundle cohomology on the spectral curve $c_{l o c}$ is a much more straightforward procedure than the analogous procedure for the M5-brane worldvolume theory. The latter would involve explicitly understanding the intermediate Jacobian of the $M 5$, its moduli dependence, its theta divisors, and then picking the 'right' theta divisor [66].

\section{Paper Layout}

Let us discuss the layout of this paper. The reader interested only in new results about instanton prefactors can skip to sections [5] and later.

In section $[5$ we propose that the seven-brane moduli dependence of a component of $A(\phi)$ can be computed via a study of the cohomology of a line bundle $\mathcal{L}_{A}$ on a spectral curve $c_{l o c}$, which exists even when there is not a heterotic dual. We discuss the relation of this method to heterotic duals and also the M-theory and type IIb limits. We briefly describe the topological computations needed to be done in order to set up the prefactor computation. We describe in detail how the compact realization of $\mathcal{C}_{l o c}$ in a natural ambient space aids the computation. In section 6 we perform a scan for instanton physics in a broad class of F-theory $E_{6}$ GUTs, utilizing the entire KreuzerSkarke database of three dimensional toric varieties as F-theory bases $B_{3}$. In section 7 we examine the relationship between points of $E_{8}$ enhancement and superpotential zeroes in generality. In section 8 we discuss in detail three examples of instanton prefactors in $E_{6}$ GUTs, setting up the prefactor computation as described in section 5.2. The first example has a heterotic dual, while the last two do not. In appendix A we resolve an $E_{6}$ GUT in F-theory and present the compact Higgs bundle spectral cover as a surface in the resolved fourfold. In appendix $B$ we give a non-toric description of the base geometry in the second and third examples of section 8 .

Sections 2, 3, and 4 primarily serve to remind the reader of important details of worldsheet instantons in the heterotic string and M5-instantons in F-theory which will 
be necessary for framing the new results in this work. In section 2 we review facts about heterotic worldsheet instanton zero modes. The instanton prefactor includes the Pfaffian of a chiral Dirac operator which depends on the gauge bundle $V$ and therefore it's moduli. It can be computed in an algebraic description in terms of the moduli which determine a spectral curve $c_{H e t}$ and appear as complex structure (7-brane) moduli in F-theory duals. We discuss the relationship between the prefactor and a theta divisor on the Jacobian $\mathcal{J}\left(c_{\mathrm{Het}}\right)$. In section 3 we review the spectral divisor formalism for describing gauge degrees of freedom in F-theory and present our compact realization of the Higgs bundle spectral cover $\mathcal{C}_{l o c}$. We review details of the M5-brane worldvolume theory and discuss the importance of Fermi zero modes and the chiral 2-form for instanton corrections. In section 4 we describe the relationship between heterotic worldsheet instantons and their dual M5-instantons in F-theory.

\subsection{Notational Conventions}

For convenience, let us collect the notation used throughout the paper. We will address the notation of each section in order.

In section 2 we use the following notation to describe $d=4 \mathcal{N}=1$ heterotic compactifications with worldsheet instanton corrections. The heterotic Calabi-Yau threefold $Z_{H e t}$ is an elliptic fibration $\pi_{H e t}: Z_{H e t} \rightarrow B_{2}$. The holomorphic vector bundle $V$ breaks one $E_{8}$ factor down to some gauge group $G_{4 d}$, often $E_{6}$ in examples. We realize $Z_{H e t}$ as a Weierstrass model, specified as a hypersurface in a bundle over $B_{2}$ whose fiber is $\mathbb{P}_{1,2,3}$ with homogeneous coordinates $[v, x, y]$. The bundle $V$ is specified by a spectral cover $\mathcal{C}_{\text {Het }}$ and a line bundle $\mathcal{N}_{\text {Het }}$ on it. $\mathcal{C}_{\text {Het }}$ is a multi-sheeted cover with covering $\operatorname{map} p_{\text {Het }}: \mathcal{C}_{\text {Het }} \rightarrow B_{2}$.

We wrap a worldsheet instanton on a curve $\Sigma$ in $B_{2}$. The component of the instanton prefactor we study vanishes 7 if and only if $h^{i}\left(\Sigma,\left.V\right|_{\Sigma} \otimes \mathcal{O}_{\Sigma}(-1)\right) \neq 0 . \mathcal{E} \equiv \pi_{H e t}^{-1} \Sigma$ is an elliptic surface, and $\left.V\right|_{\mathcal{E}}$ is specified as a spectral curve $\left.c_{H e t} \equiv \mathcal{C}_{H e t}\right|_{\mathcal{E}}$ and a line bundle $\left.\mathcal{N}_{c, \text { Het }} \equiv \mathcal{N}_{\text {Het }}\right|_{\mathcal{E}} . \quad c_{\text {Het }}$ is a multi-sheeted cover with covering map $p_{c, \text { Het }}: c_{\text {Het }} \rightarrow$ $\Sigma$. The condition regarding prefactor zeroes can be equivalently stated in terms of $h^{i}\left(c_{H e t}, \mathcal{N}_{c, H e t} \otimes p_{c, H e t}^{*} K_{\Sigma}^{1 / 2}\right) \equiv h^{i}\left(c_{H e t}, \mathcal{L}_{A}\right)^{8}$. This cohomology can be computed via a Koszul sequence from $\mathcal{E}$. Moduli dependence enters through the defining equation $f_{c_{H e t}}$ appearing as the injective map in the Koszul sequence.

In section 3 we use the following notation to describe $d=4 \mathcal{N}=1$ compactifications of F-theory with M5-instanton corrections. The F-theory Calabi-Yau fourfold $Y_{4}$ is an elliptic fibration $\pi: Y_{4} \rightarrow B_{3}$. We specify $Y_{4}$ as a hypersurface in a $\mathbb{P}_{1,2,3}$ bundle over $B_{3}$. We wrap a GUT 7-brane on a divisor $B_{2}$ which is a component of the discriminant in $B_{3}$. We study a spectral divisor $\mathcal{C}_{F}$ inside $Y_{4}$ which is the cylinder in the case of heterotic duality and is useful for specifying a dictionary between local and global Ftheory models. A chirality inducing M-theory flux $G_{4}$ can be determined from a choice of line bundle $\mathcal{N}_{F}$ on $\mathcal{C}_{F}$. More specifically, $c_{1}\left(\mathcal{N}_{F}\right)$ yields a $(1,1)$-form whose push forward into $Y_{4}$ determines $G_{4}$ up to a specific half-integral shift that we describe in great detail. The line bundle $\mathcal{N}_{F}$ itself can carry more information than its first Chern class if $\mathcal{C}_{F}$

\footnotetext{
${ }^{7}$ Since $\Sigma$ is a $\mathbb{P}^{1}$, we may write $\mathcal{O}_{\Sigma}(-1)$ for $K_{\Sigma}^{1 / 2}$ throughout.

${ }^{8}$ Throughout, we use $\mathcal{L}_{A}$ to denote the line bundle on a spectral curve whose cohomology controls the prefactor $A$, regardless of whether that curve is $c_{H e t}$ or $c_{l o c}$.
} 
is not simply connected and, in such cases, this extra information pushes forward to extra data of $C_{3}$ that is not determined by $G_{4} \cdot \mathcal{C}_{F}$ is singular in $Y_{4}$ but becomes a smooth divisor $\tilde{\mathcal{C}}_{F}$ in the resolved fourfold $\tilde{Y}_{4}$. The gauge theory on the GUT stack can be described in terms of a Higgs bundle spectral cover $\mathcal{C}_{\text {loc }}$ and a line bundle $\mathcal{N}_{\text {loc }}$ on it. $\mathcal{C}_{l o c}$ is a multi-sheeted cover with covering map $p_{l o c}: \mathcal{C}_{l o c} \rightarrow B_{2}$ and can be obtained from the spectral divisor as $\mathcal{C}_{\text {loc }}=\left.\tilde{\mathcal{C}}_{F}\right|_{\pi^{*} B_{2}}$. The line bundle $\mathcal{N}_{\text {loc }}$ can be obtained via restriction $\left.\mathcal{N}_{\text {loc }} \equiv \mathcal{N}_{F}\right|_{\mathcal{C}_{\text {loc }}}$. We show that $\mathcal{C}_{\text {loc }}$ can also be realized as a divisor in an auxiliary elliptic Calabi-Yau threefold $Z_{3, F}$ with projection map $\pi_{3, F}: Z_{3, F} \rightarrow B_{2}$. The threefold $Z_{3, F}$ and spectral cover $\mathcal{C}_{\text {loc }}$ sit inside the resolution of the GUT singular locus and are isomorphic to the threefold $Z_{H e t}$ and spectral cover $\mathcal{C}_{H e t}$ when a heterotic dual exists. $Z_{3, F}$ and $\mathcal{C}_{l o c}$ also exist in the absence of a heterotic dual, however.

We consider instantons in F-theory as a limit of instantons in $d=3 \mathcal{N}=2$ compactifications of M-theory. In M-theory on $Y_{4}$, we consider M5-instantons on divisor $\pi^{*} D 3$ for surfaces $D 3$ in $B_{3}$, where the instanton in F-theory is a D3-instanton on D3. From the perspective of the M5-brane worldvolume theory, the prefactor of an M5-instanton correction is the partition function $\mathcal{Z}_{\phi}$ of the chiral 2-form field $\phi_{2}$. It is a $\theta$ function on the intermediate Jacobian $\mathcal{J}(M 5)$. Thought of as ED3-instantons in type IIb, we study the component of the prefactor controlled by bundle cohomology on 3-7 intersection curves.

In section 4 we discuss instantons under heterotic / F-theory duality. In such a case, $B_{3}$ is a $\mathbb{P}^{1}$ fibration over $B_{2}$, so that $Y_{4}$ is globally $K 3$-fibered over $B_{2}$. One must also take the stable degeneration limit, in which the $K 3$-fibration splits into two $d P_{9}$-fibrations glued along a common elliptic fibration, the heterotic threefold $Z_{\text {Het }}$. We focus on one $d P_{9}$-fibration $Y_{4}^{\prime}$. Its base is $\mathbb{P}^{1}$-fibered and the two sections (copies of $B_{2}$ ) are the GUT stack at $Z=0$ and the heterotic threefold base $B_{2}$ at $W=0$, where $(Z, W)$ are the homogeneous coordinates on the $\mathbb{P}^{1}$ fiber. In the case of heterotic / F-theory duality, the spectral divisor $\mathcal{C}_{F}$ is the cylinder $\mathcal{C}_{c y l}$, which is a $\mathbb{P}^{1}$ fibration over $\mathcal{C}_{\text {Het }}=\mathcal{C}_{\text {loc }}$. The associated projection map(s) is $p_{c y l} . \mathcal{C}_{c y l}$ is equipped with a line bundle $\mathcal{N}_{c y l}$ and the pair $\left(\mathcal{C}_{c y l}, \mathcal{N}_{c y l}\right)$ maps to $\left(\mathcal{C}_{\text {Het }}, \mathcal{N}_{\text {Het }}\right)$ or $\left(\mathcal{C}_{\text {loc }}, \mathcal{N}_{\text {loc }}\right)$ upon restriction to $W=0$ and $Z=0$, respectively. Thus the cylinder and its line bundle give a map for translating data from the heterotic geometry to the local $E_{6}$ geometry in F-theory.

A worldsheet instanton on $\Sigma$ is dual to an M5-instanton on the pullback of $\Sigma$ under the $K 3$-fibration. Focusing on the zero modes of only the bundle $V$ corresponds to studying one of the $d P_{9}$ fibers in the stable degeneration limit, so we study $M 5 \equiv \rho^{*} \Sigma$ where $\rho: Y_{4}^{\prime} \rightarrow B_{2} . \quad M 5$ is itself an elliptic fibration $\pi_{M 5}: M 5 \rightarrow D 3$ and in the case of duality $D 3$ is $\mathbb{P}^{1}$-fibered $\nu: D 3 \rightarrow \Sigma$. Restricting the cylinder, spectral covers, and associated bundles to the $M 5$ gives a miniature version of the cylinder, a surface $c_{c y l}$ with bundle $\mathcal{N}_{M 5, c y l}$ which restricts to pairs of spectral curves $\left(c_{\text {Het }}, \mathcal{N}_{c, H e t}\right)$ and $\left(c_{\text {loc }}, \mathcal{N}_{c, l o c}\right)$ at $W=0$ and $Z=0$ respectively. $c_{c y l}$ is a multi-sheeted cover of $D 3$ with $p_{M 5}: c_{c y l} \rightarrow D 3 . \quad c_{H e t}$ and $c_{l o c}$ are both multi-sheeted covers of $\Sigma$, the first in the heterotic geometry and the inside the GUT singular locus. The $\mathcal{L}_{A}$ cohomologies on $c_{l o c}$ and $c_{H e t}$ are isomorphic, and thus the prefactor can be determined by studying cohomology on either curve.

In section 5 we discuss the physics and interpretation of the moduli-dependent cohomology $h^{i}\left(c_{l o c}, \mathcal{N}_{c, l o c} \otimes p_{c, l o c}^{*} K_{\Sigma}^{1 / 2}\right) \equiv h^{i}\left(c_{l o c}, \mathcal{L}_{A}\right)$ and conjecture that it (and similar cohomologies of associated bundles $\wedge^{k} V$ ) can be used to determine the instanton pref- 
actor in terms of algebraic seven-brane moduli. $\left[c_{l o c}\right]$ is a divisor in an elliptic surface $\mathcal{E} \equiv \pi_{3, F}^{-1} \Sigma$ of class $n s+r F$ where $n=r k(V), s$ is the section of $\mathcal{E}, r \equiv \eta \cdot B_{2} \Sigma$, $\eta=6 c_{1, B_{2}}+N_{B_{2} \mid B_{3}}$, and $F$ is the fiber class of $\mathcal{E}$. $\mathcal{L}_{A}$ is a bundle on $c_{l o c}$ and is obtained via restriction from a line bundle (also called $\mathcal{L}_{A}$, abusing notation) on $\mathcal{E}$ of first Chern class $\left.c_{1}\left(\mathcal{L}_{A}\right)=\left(\lambda+\frac{1}{2}\right) n s+\left[r\left(\frac{1}{2}-\lambda\right)+\chi\left(\frac{1}{2}+n \lambda\right)-1\right)\right] F$, where $\chi \equiv c_{1, B_{2}} \cdot B_{2} \Sigma$ and $\lambda$ is a flux parameter, integral or half-integral depending on $n$. Given the data $\{n, \lambda, r, \chi\}$ the mathematical computation can go forward in a way identical to the heterotic com-

putations of [59]. $h^{i}\left(c_{l o c}, \mathcal{N}_{c, l o c} \otimes p_{c, l o c}^{*} K_{\Sigma}^{1 / 2}\right) \equiv h^{i}\left(c_{l o c}, \mathcal{L}_{A}\right)$ exists whether or not there is a heterotic dual.

\section{Heterotic Worldsheet Instantons}

We begin in this section by reviewing background material on worldsheet instantons in $E_{8} \times E_{8}$ heterotic strings. This will allow us to set some notation and frame the discussion in a way that closely parallels our subsequent study of M5 instantons.

We consider the compactification of $E_{8} \times E_{8}$ heterotic strings on an elliptically fibered Calabi-Yau 3 -fold $Z_{H e t}$ with section over a base $B_{2}$

$$
\pi_{H e t}: Z_{H e t} \rightarrow B_{2}
$$

For simplicity, we restrict our attention to one $E_{8}$ factor and introduce an $S U(3)$ bundle $V$ to break this $E_{8}$ down to $E_{6}$. Insights into instantons gained in this case can be extended to other GUT groups via standard techniques. Our interest is in the superpotential couplings generated by worldsheet instantons that wrap curves in $B_{2}$ with particular attention to their dependence on $V$.

The 3-fold $Z_{\text {Het }}$ can be realized as a Weierstrass model, which we specify in this paper as a hypersurface inside a $\mathbb{P}_{1,2,3}^{2}$ bundle over $B_{2}$. Letting $[v, x, y]$ denote the weighted homogeneous coordinates of the $\mathbb{P}_{1,2,3}^{2}$ fiber, we write the defining equation as

$$
y^{2}=x^{3}+f x v^{4}+g v^{6} .
$$

The bundle $V$ is constructed via a Fourier-Mukai transform from a spectral cover

$$
\mathcal{C}_{H e t}: a_{0} v^{3}+a_{2} v x+a_{3} y=0
$$

and a choice of line bundle $\mathcal{N}_{\text {Het }}$ on $\mathcal{C}_{\text {Het }}$. In writing (2.2) and (2.3) we have introduced a number of holomorphic sections on $Z_{\text {Het }}$ which are associated to the bundles

\begin{tabular}{c|c} 
Section & Bundle \\
\hline$v$ & $\mathcal{O}\left(\sigma_{H e t}\right)$ \\
$x$ & $\mathcal{O}\left(2\left[\sigma_{H e t}+c_{1, B_{2}}\right]\right)$ \\
$y$ & $\mathcal{O}\left(3\left[\sigma_{H e t}+c_{1, B_{2}}\right]\right)$ \\
$a_{0}$ & $\mathcal{O}(\eta)$ \\
$a_{2}$ & $\mathcal{O}\left(\eta-2 c_{1, B_{2}}\right)$ \\
$a_{3}$ & $\mathcal{O}\left(\eta-3 c_{1, B_{2}}\right)$.
\end{tabular}

Here, $\sigma_{H e t}$ is the divisor class of the section of $Z_{H e t}, c_{1, B_{2}}$ is shorthand for the anticanonical class of $B_{2}$, and $\eta$ is a divisor class on $B_{2}$ that we are free to choose subject to 
the condition that all bundles in (2.4) actually admit holomorphic sections. Note that we do not notationally distinguish between divisor classes on $B_{2}$ or their pullbacks to $Z_{\text {Het }}$.

To ensure that the bundle $V$ obtained from (2.3) and $\mathcal{N}_{\text {Het }}$ has structure group $S U(3)$ rather than $U(3)$ one must impose the 'traceless' condition

$$
c_{1}\left(p_{H e t *} \mathcal{N}_{H e t}\right)=0
$$

where $p_{\text {Het }}$ is the covering map

$$
p_{H e t}: \mathcal{C}_{H e t} \rightarrow B_{2}
$$

By Grothendieck-Riemann-Roch,

$$
c_{1}\left(p_{H e t *} \mathcal{N}_{H e t}\right)=p_{H e t *} c_{1}\left(\mathcal{N}_{H e t}\right)-\frac{1}{2} p_{H e t *} r_{H e t}
$$

where $r_{H e t}$ is the ramification divisor of the covering $p_{H e t}$

$$
r_{H e t}=p_{H e t}^{*} c_{1, B_{2}}-c_{1}\left(\mathcal{C}_{H e t}\right)
$$

so bundles $\mathcal{N}_{\text {Het }}$ satisfying (2.5) can be associated to divisors $\gamma_{\text {Het }}$ satisfying

$$
p_{H e t *} \gamma_{H e t}=0
$$

according to

$$
c_{1}\left(\mathcal{N}_{H e t}\right)=\lambda \gamma_{H e t}+\frac{r_{H e t}}{2}
$$

where $\lambda$ will be a half-integer whenever $r_{H e t} / 2$ is a half-integral class. The spectral cover $\mathcal{C}_{\text {Het }}$ is simply connected in general. In this case, there exists a unique divisor class $\gamma_{H e t}$ satisfying (2.9)

$$
\gamma_{H e t}=3 \sigma_{H e t}-\left(\eta-3 c_{1, B_{2}}\right)
$$

Given our Calabi-Yau $Z_{H e t}$ and bundle $V$, we pick a distinguished curve $\Sigma$ in $B_{2}$ and investigate the superpotential correction generated by worldsheet instantons wrapping $\Sigma$. This correction will vanish if the instanton does not have the right Fermi zero mode structure. In general, one has two types of zero modes; vector-like pairs of left- and right-movers that do not couple to $V$ and left-movers that do couple to $V$. We address each of these in turn.

\subsection{Fermi Zero Modes that Don't Couple to $V$}

For completeness, first recall the structure of the right-moving Fermi zero modes, which don't couple to $V$. Following [9,49, 67], one can establish that these modes are counted by

$$
2 \times\left[h^{0}\left(\Sigma, N_{\Sigma / Z_{H e t}}\right)+h^{0}(\Sigma, \mathcal{O})+h^{0}\left(\Sigma, K_{\Sigma}\right)\right]
$$

with $N_{\Sigma / Z_{H e t}}$ denoting the normal bundle of $\Sigma$ inside the heterotic Calabi-Yau $Z_{H e t}$. In this paper we always take $\Sigma$ to be a curve that sits in the section $B_{2}$ of the elliptically fibered 3 -fold $Z_{H e t}$. In that case

$$
N_{\Sigma / Z_{H e t}}=N_{\Sigma / B_{2}}+\left.K_{B_{2}}\right|_{\Sigma}=N_{\Sigma / B_{2}}+K_{\Sigma} \otimes N_{\Sigma / B_{2}}^{-1}
$$


and the Fermi zero mode spectrum is counted by

$$
2 \times\left[h^{0}\left(\Sigma, N_{\Sigma / B_{2}}\right)+h^{1}\left(\Sigma, N_{\Sigma / B_{2}}\right)+h^{0}(\Sigma, \mathcal{O})+h^{1}(\Sigma, \mathcal{O})\right]
$$

where we used Serre duality to relate $h^{0}\left(\Sigma, K_{\Sigma} \otimes N_{\Sigma / B_{2}}^{-1}\right)=h^{1}\left(\Sigma, N_{\Sigma / B_{2}}\right)$ and $h^{0}\left(\Sigma, K_{\Sigma}\right)=$ $h^{1}(\Sigma, \mathcal{O})$. The contribution from $h^{0}(\Sigma, \mathcal{O})=1$ is just the ordinary pair of 'universal' Fermi zero modes that one expects from an instanton that generates a superpotential coupling. To ensure that a superpotential coupling is truly generated, however, the remaining cohomologies must vanish

$$
0=h^{0}\left(\Sigma, N_{\Sigma / B_{2}}\right)=h^{1}\left(\Sigma, N_{\Sigma / B_{2}}\right)=h^{1}(\Sigma, \mathcal{O}) .
$$

The condition $h^{1}(\Sigma, \mathcal{O})=h^{0}\left(\Sigma, K_{\Sigma}\right)$ implies that $\Sigma$ must be a $\mathbb{P}^{1}$ while the remaining two terms indicate that the normal bundle of $\Sigma$ in $B_{2}$ must have vanishing cohomologies. On $\mathbb{P}^{1}$, this is only true for $N_{\Sigma / B_{2}}=\mathcal{O}(-1)$. Since the degree of the normal bundle of any $\mathbb{P}^{1}$ inside a Calabi-Yau 3 -fold is -2 , we avoid extra right-handed Fermi zero modes only when

$$
\Sigma=\mathbb{P}^{1} \quad N_{\Sigma / Z_{H e t}}=\mathcal{O}(-1) \oplus \mathcal{O}(-1) .
$$

The second condition will be true whenever

$$
N_{\Sigma / B_{2}}=\mathcal{O}(-1)
$$

Note that such an instanton will always be isolated in the sense that $N_{\Sigma / Z_{H e t}}$ has no global sections. Such instantons do not have non-universal bosonic zero modes, which would include bosonic deformation modes transverse to the instanton.

\subsection{Fermi Zero Modes that Couple to $V$ : the Pfaffian}

We turn now to the worldsheet fermions that couple to $V$. Our interest in this paper is to study the dependence of instanton-generated couplings on the moduli of $V$. Integrating out worldsheet fermions that couple to $V$ yields a prefactor proportional to the Pfaffian of the Dirac operator $D_{-}$on $\Sigma$

$$
W \sim \operatorname{Pfaff}\left(\Sigma, D_{-, V_{\Sigma}}\right) e^{i \int_{\Sigma} B}
$$

associated to the bundle

$$
V_{\Sigma}=\left.V\right|_{\Sigma}
$$

It is well-known that only the product $\operatorname{Pfaff}\left(\Sigma, D_{-}, V_{\Sigma}\right) e^{i \int_{\Sigma} B}$ is gauge invariant; the individual terms of the product are not. Rather, $B$ shifts under a gauge transformation parametrized by $\epsilon$ according to

$$
B \rightarrow B+\operatorname{tr} \epsilon F
$$

while the Pfaffian transforms in the right way to cancel this. This is the famous statement that the Pfaffian is not a function but rather a section of a suitable line bundle on the moduli space of gauge fields $V_{\Sigma}$.

We can describe $V_{\Sigma}$ and its moduli nicely by restricting the spectral data of $V$ to the elliptic fibration $\mathcal{E}$ over $\Sigma$

$$
\mathcal{E}=\pi_{H e t}^{*} \Sigma \quad \pi_{\mathcal{E}}: \mathcal{E} \rightarrow \Sigma .
$$


Defining

$$
c_{\text {Het }}=p_{\text {Het }}^{*} \Sigma \quad \mathcal{N}_{c, H e t}=\left.\mathcal{N}_{\text {Het }}\right|_{c_{H e t}} \quad p_{c, H e t}: c_{H e t} \rightarrow B_{2}
$$

we have that $V_{\Sigma}$ is simply the pushforward of $\mathcal{N}_{c, \text { Het }}$

$$
V_{\Sigma}=p_{c, H e t *} \mathcal{N}_{c, H e t} .
$$

In general, Pfaff $\left(\Sigma, D_{-, V_{\Sigma}}\right)$ depends on the choice of curve $c_{H e t}$ in $\pi_{H e t}^{*} \Sigma$ as well as the choice of line bundle $\mathcal{N}_{c, \text { Het }}$ on $c_{H e t}$. It vanishes whenever $D_{-, V_{\Sigma}}$ admits zero modes or, equivalently, whenever $H^{0}\left(\Sigma, K_{\Sigma}^{1 / 2} \otimes V_{\Sigma}\right)$ is nontrivial. Cohomologies of $K_{\Sigma}^{1 / 2} \otimes V_{\Sigma}$ can be related to cohomologies on $c_{H e t}$

$$
H^{p}\left(\Sigma, K_{\Sigma}^{1 / 2} \otimes V_{\Sigma}\right)=H^{p}\left(c_{H e t}, p_{c, H e t}^{*} K_{\Sigma}^{1 / 2} \otimes \mathcal{N}_{c, H e t}\right)
$$

by a Leray spectral sequence. Note that there is no ambiguity in the choice of spin structure $K_{\Sigma}^{1 / 2}$ since we always take $\Sigma=\mathbb{P}^{1}$. Further, since $c_{1}\left(V_{\Sigma}\right)=0$ we have that

$$
0=\chi\left(\Sigma, K_{\Sigma} \otimes V_{\Sigma}\right)=\chi\left(c_{H e t}, p_{c, H e t}^{*} K_{\Sigma}^{1 / 2} \otimes \mathcal{N}_{c, H e t}\right)
$$

so that the bundle $p_{c, H e t}^{*} K_{\Sigma}^{1 / 2} \otimes \mathcal{N}_{c, \text { Het }}$ has degree $g_{c_{H e t}}-1$. The full space of possible $\left[p_{c, \text { Het }}^{*} K_{\Sigma}^{1 / 2} \otimes \mathcal{N}_{c, H e t}\right]$ 's, then comprises the degree $g-1$ Jacobian $\mathcal{J}_{g-1}\left(c_{H e t}\right)$ of the covering curve $c_{H e t}$. In general, very few bundles on $\mathcal{J}_{g-1}\left(c_{H e t}\right)$ will arise from the restriction of a bundle $\mathcal{N}_{\text {Het }}$ on $\mathcal{C}_{\text {Het }}$. Let us forget about this for a moment, though, and consider the Pfaffian of a generic $S U(3)$ bundle $V_{\Sigma}$ on $\Sigma$ constructed from a fixed cover $c_{H e t}$ and an arbitrary choice of degree $g_{c_{H e t}}-1$ bundle $p_{c, H e t}^{*} K_{\Sigma}^{1 / 2} \otimes \mathcal{N}_{c, H e t}$. We consider first the dependence of this Pfaffian on $p_{c, H e t}^{*} K_{\Sigma}^{1 / 2} \otimes \mathcal{N}_{c, H e t}$ before using the fact that Jacobians vary holomorphically in families to see how it depends on the moduli of $c_{\text {Het }}$.

It is well known that the dependence of $\operatorname{Pfaff}\left(\Sigma, D_{-, V_{\Sigma}}\right)$ on $\mathcal{N}_{c, H e t}$ is uniquely fixed by its vanishing locus, namely those choices of $\mathcal{N}_{c, \text { Het }}$ such that $\mathcal{N}_{c, \text { Het }} \otimes p_{c, H e t}^{*} K_{\Sigma}^{1 / 2}$ admits holomorphic sections. We will review this uniqueness result explicitly in a moment but first let us use it to relate the $\mathcal{N}_{c, H e t}$-dependence of $\operatorname{Pfaff}\left(\Sigma, D_{-, V_{\Sigma}}\right)$, which is a partition function of free fermions coupled to a non-Abelian vector bundle $V_{\Sigma}$, to the more familiar problem of a partition function of free fermions coupling to an Abelian bundle. If we choose a spin structure $K_{c_{H e t}}^{1 / 2}$ on $c_{H e t}$, we can rewrite the degree $g_{c_{H e t}}-1$ bundle of interest as

$$
\mathcal{N}_{c, H e t} \otimes p_{c, H e t}^{*} K_{\Sigma}^{1 / 2}=K_{c_{H e t}}^{1 / 2} \otimes \mathcal{L}_{\Gamma}
$$

where

$$
\begin{aligned}
\mathcal{L}_{\Gamma} & =\mathcal{N}_{c, H e t} \otimes p_{c, H e t}^{*} K_{\Sigma}^{1 / 2} \otimes K_{c_{H e t}}^{1 / 2} \\
& =\mathcal{N}_{c, \text { Het }} \otimes \mathcal{O}\left(-R_{\text {Het }} / 2\right) .
\end{aligned}
$$

Here, $R_{\text {Het }}$ is the ramification divisor of the covering $p_{c, \text { Het }}$ and $\mathcal{O}\left(-R_{\text {Het }} / 2\right)$ is a square root of $\mathcal{O}\left(-R_{H e t}\right)$ that is determined by the choice of spin structure $K_{c_{H e t}}^{1 / 2}$. All we have done here is use $K_{c_{H e t}}^{1 / 2}$ to define a map to the degree 0 part of the Jacobian

$$
\mathcal{J}_{g-1}\left(c_{H e t}\right) \rightarrow \mathcal{J}_{0}\left(c_{H e t}\right)
$$


that takes

$$
\mathcal{N}_{c, H e t} \otimes p_{c, H e t}^{*} K_{\Sigma}^{1 / 2} \mapsto \mathcal{L}_{\Gamma}
$$

where we emphasize that $\mathcal{L}_{\Gamma}$ has degree 0 . Roughly speaking, one can think of $\mathcal{L}_{\Gamma}$ as the restriction of the $\lambda \gamma_{H e t}$ piece of $\mathcal{N}_{\text {Het }}(2.10)$ to $c_{H e t}$. Because $\lambda \gamma_{H e t}$ and $r_{H e t} / 2$ are both non-integral in general, the separation of $c_{1}\left(\mathcal{N}_{H e t}\right)$ in (2.10) cannot be lifted to a split of $\mathcal{N}_{\text {Het }}$ into the tensor product of line bundles $\mathcal{O}\left(\lambda \gamma_{\text {Het }}\right) \otimes \mathcal{O}\left(r_{\text {Het }} / 2\right)$. Upon restricting to $c_{H e t}$, though, $\left.\gamma\right|_{c_{H e t}} \equiv \Gamma_{H e t}$ and $\left.r_{H e t}\right|_{c_{H e t}}=R_{H e t}$ are even divisors whose corresponding line bundles, $\mathcal{O}\left(\Gamma_{H e t}\right)$ and $\mathcal{O}\left(R_{H e t}\right)$, admit square roots, $\mathcal{O}\left(\Gamma_{\text {Het }} / 2\right)$ and $\mathcal{O}\left(R_{\text {Het }} / 2\right)$. There are $2^{2 g_{c_{H e t}}}$ choices for how we take these square roots corresponding to the $2^{2 g_{c_{H e t}}}$ choices of spin structure $K_{c_{H e t}}^{1 / 2}$.

Now, the $\mathcal{L}_{\Gamma}$-dependence of our desired Pfaffian is uniquely fixed by the fact that it vanishes when $H^{0}\left(c_{H e t}, K_{c_{H e t}}^{1 / 2} \otimes \mathcal{L}_{\Gamma}\right)$ is nontrivial. This is precisely the vanishing locus of the partition function of a free fermion on $c_{H e t}$ coupled to $\mathcal{L}_{\Gamma}$ and hence

$$
\operatorname{Pfaff}\left(\Sigma, D_{-, V_{\Sigma}}\right) \sim \operatorname{Pfaff}\left(c_{H e t}, D_{-, \mathcal{L}_{\Gamma}}\right)
$$

where it is understood that the map from $V_{\Sigma}$ to $\left(c_{H e t}, \mathcal{L}_{\Gamma}\right)$ depends on the choice of spin structure on $c_{\text {Het }}$.

Given this equivalence, we could have addressed the problem of studying $\operatorname{Pfaff}\left(\Sigma, D_{-, V_{\Sigma}}\right)$ by computing $\operatorname{Pfaff}\left(c_{H e t}, D_{-, \mathcal{L}_{\Gamma}}\right)$ directly. The latter object is well studied and depends on $\mathcal{L}_{\Gamma} \in \mathcal{J}_{0}\left(c_{H e t}\right)$ not as a holomorphic function but as a holomorphic section of a line bundle $\hat{L}$ on $\mathcal{J}_{0}\left(c_{H e t}\right)$. This reflects the fact that $\operatorname{Pfaff}\left(c_{H e t}, D_{-, \mathcal{L}_{\Gamma}}\right)$ is not gauge invariant, a welcome property because it must violate gauge invariance in the right way to cancel the anomalous transformation law of the $B$-field (2.20). On these grounds alone one can argue that $\frac{1}{2 \pi} c_{1}(\hat{L})=\omega$ must yield a principal polarization [66] of the Abelian variety $\mathcal{J}_{0}\left(c_{H e t}\right)$. This is almost enough to determine $\hat{L}$ but not quite because $\mathcal{J}_{0}\left(c_{\text {Het }}\right)$ is not simply connected. One must further specify the holonomies of $\hat{L}$ around the 1 -cycles of $\mathcal{J}_{0}\left(c_{\mathrm{Het}}\right)$ and, as discussed in [66], there is no unique way to do this. Instead, there are $2^{2 g_{c_{H e t}}}$ choices of bundle $\hat{L}_{m}$ on $\mathcal{J}_{0}\left(c_{H e t}\right)$ with $\frac{1}{2 \pi} c_{1}\left(\hat{L}_{m}\right)=\omega$, one for each choice of spin structure on $c_{\text {Het }}$. Each of these $2^{2 g_{c_{H e t}}}$ bundles, $\hat{L}_{m}$, admits a unique holomorphic section $\theta_{m}$ whose vanishing specifies a theta divisor $\Theta_{m}$. It remains to determine which line bundle is the 'right' one. Given that, $\operatorname{Pfaff}\left(c_{H e t}, D_{-, \mathcal{L}_{\Gamma}}\right)$ will be proportional to the corresponding $\theta_{m}$.

As we review in section 3.2 .2 , one encounters a completely analogous problem when studying the partition function of the chiral 2-form on an M5 instanton. A general approach for determining the 'right' $\theta_{m}$, applicable both for this situation and our heterotic Pfaffians, is outlined in [66]. While straightforward, this procedure is not easy to apply in practice. For our heterotic worldsheet instantons it is actually unnecessary because we have more information available than the transformation properties of the desired partition function: we know its vanishing locus precisely and in a way that doesn't have an explicit dependence on spin structure. As we discuss in section 5.1, it is our hope that similar considerations can apply to M5 instantons as well.

Let us review why the Pfaffian doesn't have an explicit dependence on a choice of spin structure. Recall that $\operatorname{Pfaff}\left(\Sigma, D_{-, V_{\Sigma}}\right)$ vanishes whenever $H^{0}\left(c_{H e t}, \mathcal{N}_{c, \text { Het }} \otimes p_{c, \text { Het }}^{*} K_{\Sigma}^{1 / 2}\right)$ is nontrivial. The collection of degree $g_{c_{H e t}}-1$ line bundles $\mathcal{L}_{A}=\mathcal{N}_{c, H e t} \otimes p_{c, H e t}^{*} K_{\Sigma}^{1 / 2}$ with section is remarkable in that it is a theta divisor of the Abelian variety $\mathcal{J}_{g-1}\left(c_{H e t}\right)$. 
Because of its special origin, this theta divisor is distinguished among the others on $\mathcal{J}_{g-1}\left(c_{H e t}\right)$ and it is typically referred to as the Riemann Theta divisor, $\Theta_{R}$. Like all theta divisors, it specifies a line bundle on $\mathcal{J}_{g-1}\left(c_{\text {Het }}\right)$ whose first Chern class is a principal polarization and admits a unique holomorphic section $\theta_{R}$ that vanishes along $\Theta_{R}$. The $\mathcal{N}_{c_{H e t}}$-dependence of $\operatorname{Pfaff}\left(\Sigma, D_{-, V_{\Sigma}}\right)$ is therefore determined entirely by its vanishing locus up to an overall constant

$$
\operatorname{Pfaff}\left(\Sigma, D_{-, V_{\Sigma}}\right) \sim \theta_{R}\left(\mathcal{N}_{c, H e t} \otimes p_{c_{H e t}}^{*} K_{\Sigma}^{1 / 2}\right) .
$$

When we rewrite our bundle in terms of $\mathcal{L}_{\Gamma}$ as in (2.26), all we do is obtain translates of $\theta_{R}$ on $\mathcal{J}_{0}\left(c_{H e t}\right)$

$$
\operatorname{Pfaff}\left(\Sigma, D_{-, V_{\Sigma}}\right) \sim \theta_{R}\left(K_{c_{H e t}}^{1 / 2} \otimes \mathcal{L}_{\Gamma}\right) \equiv \theta_{-K_{c_{H e t}}^{1 / 2}}\left(\mathcal{L}_{\Gamma}\right)
$$

The translate $\theta_{-K_{c_{H e t}}^{1 / 2}}$ defines a theta divisor on $\mathcal{J}_{0}\left(c_{H e t}\right)$ and is the 'right' $\theta_{m}$ for the

choice of spin structure $K_{c_{H e t}}^{1 / 2}$. Said differently, given a choice of spin structure the Pfaffian is specified by the theta divisor $\Theta_{m}$ of $\mathcal{J}_{0}\left(c_{H e t}\right)$ which is a translate of $\Theta_{R}$ by $K_{c_{H e t}}^{1 / 2}$.

We see that the introduction of a spin structure on $c_{H e t}$ is completely unnecessary to determine the dependence of $\operatorname{Pfaff}\left(\Sigma, D_{-, V_{\Sigma}}\right)$ on the choice of line bundle on $c_{H e t}$. Rather, it is an auxiliary operation that arises when we try to mimic the separation (2.10) at the level of line bundles on $c_{H e t}$. Even then, however, the Pfaffian is ultimately determined entirely by its vanishing structure which makes no direct reference to any of this. One hopes that M5 instantons can be understood in a similar way, allowing us to get a handle on vanishing properties without following through the general procedure of [66]. An approach like this is guaranteed to work when a heterotic dual exists and we would like to suggest that it may apply more generally as well.

\subsection{Moduli Dependence of the Pfaffian}

Our previous considerations focused on the vanishing structure of the Pfaffian and determining the right $\theta$ function for controlling its variation as one moves in the moduli space of bundles on $c_{H e t}$. In general, only a discrete set of bundles $\mathcal{N}_{c, H e t} \in \mathcal{J}_{g-1}\left(c_{H e t}\right)$ will arise as the restriction of bundles $\mathcal{N}_{\text {Het }}$ on $\mathcal{C}_{\text {Het }}$. This makes the previous discussion useful for comparisons with M5 instantons but not very relevant for determining the moduli dependence of the instanton prefactor. The latter requires an understanding of how $\operatorname{Pfaff}\left(\Sigma, D_{-, V_{\Sigma}}\right)$ depends on the algebraic moduli appearing in the defining equation of $c_{H e t}$ and it is this dependence that was computed in a number of examples in [59,68,69]. As explained in [49], the Pfaffian of a bundle $\mathcal{L}_{A}$ on a curve $c_{H e t}$ can be obtained as the determinant of a map $f$ appearing in an exact sequence of the form

$$
0 \rightarrow H^{0}\left(c_{H e t}, \mathcal{L}_{A}\right) \rightarrow W_{1} \stackrel{f}{\rightarrow} W_{2} \rightarrow H^{1}\left(c_{H e t}, \mathcal{L}_{A}\right) \rightarrow 0
$$

In [59, 68, 69, sequences of this type were obtained by studying the Koszul complex relating line bundles on $c_{H e t}$ to those on $\mathcal{E}=\pi_{H e t}^{*} \Sigma$, the elliptic fibration over $\Sigma$ that contains $c_{\text {Het }}$. A Koszul approach is made possible because our bundle of interest

$$
\mathcal{L}_{A}=\mathcal{N}_{c, H e t} \otimes p_{c, H e t}^{*} K_{\Sigma}^{1 / 2}
$$


is the restriction of a line bundle $\mathcal{L}_{\mathcal{E}}$ on $\mathcal{E}$

$$
\mathcal{L}_{\mathcal{E}}=\left.\mathcal{O}_{Z_{H e t}}(\lambda \gamma+r / 2)\right|_{\mathcal{E}} \otimes \pi_{\mathcal{E}}^{*} K_{\Sigma}^{1 / 2}
$$

The Koszul sequence

$$
\left.0 \rightarrow \mathcal{L}_{A} \otimes \mathcal{O}\left(-c_{H e t}\right) \stackrel{f_{c_{H e t}}}{\longrightarrow} \mathcal{L}_{A} \rightarrow \mathcal{L}_{A}\right|_{c_{H e t}} \rightarrow 0
$$

and leads to the long exact cohomology sequence

$$
\begin{aligned}
0 & \rightarrow H^{0}\left(\mathcal{E}, \mathcal{L}_{\mathcal{E}} \otimes \mathcal{O}\left(-c_{H e t}\right)\right) \rightarrow H^{0}\left(\mathcal{E}, \mathcal{L}_{\mathcal{E}}\right) \rightarrow H^{0}\left(c_{H e t}, \mathcal{L}_{c}\right) \\
& \rightarrow H^{1}\left(\mathcal{E}, \mathcal{L}_{\mathcal{E}} \otimes \mathcal{O}\left(-c_{H e t}\right)\right) \stackrel{f}{\rightarrow} H^{1}\left(\mathcal{E}, \mathcal{L}_{\mathcal{E}}\right) \rightarrow H^{1}\left(c_{H e t}, \mathcal{L}_{c}\right) \\
& \rightarrow H^{1}\left(\mathcal{E}, \mathcal{L}_{\mathcal{E}} \otimes \mathcal{O}\left(-c_{H e t}\right)\right) \rightarrow H^{1}\left(\mathcal{E}, \mathcal{L}_{\mathcal{E}}\right) \rightarrow 0
\end{aligned}
$$

When $H^{0}\left(\mathcal{E}, \mathcal{L}_{\mathcal{E}}\right)=0$ and $H^{1}\left(\mathcal{E}, \mathcal{L}_{\mathcal{E}} \otimes \mathcal{O}\left(-c_{H e t}\right)\right)=0$ we have a sequence of precisely the form (2.33) and the moduli dependence of $\operatorname{Pfaff}\left(c_{H e t}, D_{-, \mathcal{L}_{\Gamma}}\right)$ is captured by the determinant of the map $f$. A matrix representative for $f$ is often straightforward to compute so one can obtain very explicit expressions. Intriguingly, these often exhibit a very high degree of structure that we would like to better understand. To us, the structures are indicative of beautiful physics, and we will study them in later sections.

\section{$3 \quad$ F-theory and M5 Instantons}

We now turn to F-theory compactified on an elliptically fibered Calabi-Yau 4-fold $Y_{4}$ with section over a base $B_{3}$

$$
\pi: Y_{4} \rightarrow B_{3} .
$$

We review and clarify the spectral divisor formalism for constructing $G$-fluxes in these compactifications with an eye toward properly specifying the 3 -form, $C_{3}$. This requires keeping track of more than the homology class of a holomorphic surface that is Poincare dual to $G_{4}$. We emphasize the specification of a line bundle on the spectral divisor as a starting point and carefully construct the bundle by which it should be twisted to yield a properly quantized $G$-flux. To make precise how these $G$-fluxes communicate to the local GUT model on $B_{2}$, we also describe a careful study of the compact surface that emerges from $Y_{4}$, or more properly its resolution, and plays the role of the Higgs bundle spectral cover $\mathcal{C}_{l o c}$. Previous work [32] has shown how the non-compact spectral cover of local models arises from the restriction of the spectral divisor to $\pi^{*} B_{2}$. We study the full compact surface $\mathcal{C}_{\text {loc }}$ and demonstrate that it is naturally described as a hypersurface in an auxiliary elliptically fibered Calabi-Yau 3-fold that is isomorphic to the heterotic Calabi-Yau when a heterotic dual exists. We will see in subsequent sections that this auxiliary space nicely facilitates instanton computations. Following this discussion, we review some background on M5 instantons in preparation for subsequent sections.

\subsection{F-theory Preliminaries}

As usual, we think of F-theory as a limit of M-theory in which the elliptic fiber of $Y_{4}$ is shrunk to zero size while holding its complex structure modulus fixed. In M-theory, 
the instantons of interest come from Euclidean M5's that wrap vertical divisors of $Y_{4}$, that is divisors of the form $\pi^{*} D 3$ for surfaces $D 3$ in $B_{3}$. In taking the F-theory limit by taking two torus one-cycles to zero size in succession, the M5-instanton becomes a D4instanton in type IIA and then a D3-instanton in F-theory 9. As the notation suggests, these descend to D3-instantons in the IIB limit while they often correspond to heterotic worldsheet instantons when a heterotic dual exists. Before getting to M5's, however, let us describe our setup more carefully and set some notation.

As with the heterotic 3 -fold $Z_{H e t}$, we will realize our F-theory 4 -fold $Y_{4}$ as a hypersurface inside a $\mathbb{P}_{1,2,3}^{2}$ bundle over the base. We denote the ambient $\mathbb{P}_{1,2,3}^{2}$ bundle by $W_{5}$ and restrict our attention to 4 -folds in $W_{5}$ that exhibit an $E_{6}$ singularity along a surface $B_{2}$ in $B_{3}$. When a heterotic dual exists, $B_{2}$ will be identified with the base of $Z_{\text {Het }}$. Letting $[v, x, y]$ denote the weighted homogeneous coordinates of the $\mathbb{P}_{1,2,3}^{2}$ fiber, we write the defining equation of $Y_{4}$ in $W_{5}$ as

$$
y^{2}=x^{3}+f x z^{4} v^{4}+g z^{6} v^{6}+z^{2} v^{3}\left[b_{0} z^{3} v^{3}+b_{2} z v x+b_{3} y\right]
$$

where the various objects that appear are holomorphic sections of the indicated bundles

\begin{tabular}{c|c} 
Section & Bundle \\
\hline$v$ & $\mathcal{O}(\sigma)$ \\
$x$ & $\mathcal{O}\left(2 \sigma+2 c_{1, B_{3}}\right)$ \\
$y$ & $\mathcal{O}\left(3 \sigma+3 c_{1, B_{3}}\right)$ \\
$z$ & $\mathcal{O}\left(B_{2}\right)$ \\
$f$ & $4 \mathcal{O}\left(c_{1, B_{3}}-B_{2}\right)$ \\
$g$ & $6 \mathcal{O}\left(c_{1, B_{3}}-B_{2}\right)$ \\
$b_{m}$ & $\mathcal{O}\left([6-m] c_{1, B_{3}}-[5-m] B_{2}\right)$
\end{tabular}

Here $\sigma$ is the section of $Y_{4}$ and $c_{1, B_{3}}$ is shorthand for an anti-canonical divisor on $B_{3}$. Note that we do not distinguish between divisors on $B_{3}$ and their pullbacks to $Y_{4}$.

A complete specification of an F-theory compactification requires not just $Y_{4}$ but also a configuration of the M-theory 3 -form, $C_{3}$. One typically provides the field strength $G_{4}$ associated to this 3 -form and focuses on supersymmetric vacua where $G_{4}$ is harmonic of type $(2,2)$ and primitive. In practice, these $G$-fluxes are specified by giving their Poincare dual holomorphic surfaces in $Y_{4}$ or, more carefully, its Calabi-Yau resolution $\tilde{Y}_{4}$. Lorentz invariance requires that $G_{4}$ have 'one leg on the fiber' which is to say that it integrates to zero over any surface that contains the elliptic fiber or sits inside the section.

Our fourfold $Y_{4}$ is singular along $B_{2}$ and the physics of that singularity is captured by an 8-dimensional $E_{6}$ gauge theory on $\mathbb{R}^{3,1} \times B_{2}$. The $G$-fluxes of interest are the ones that control the spectrum of this theory. Describing them can be complicated because the Poincare dual surfaces are smooth in $\tilde{Y}_{4}$ but degenerate in the singular limit $\tilde{Y}_{4} \rightarrow Y_{4}$. A formalism has been developed for simplifying the analysis based on the notion of a spectral divisor [29, 30, 32. For our $Y_{4}$ (3.2), we define the spectral divisor $\mathcal{C}_{F}$ as the hypersurface of $Y_{4}$ given by 10

\footnotetext{
${ }^{9}$ Recall that throughout we call the instantons in F-theory $M 5$-instantons to emphasize their M-theoretic origin, keeping in mind that they are really D3-branes.

${ }^{10}$ If we define $\mathcal{C}_{F}$ by (3.4) one must take some care. When we resolve the singularities of $Y_{4}$, the proper
} 


$$
\mathcal{C}_{F}: \quad b_{0} z^{3} v^{3}+b_{2} z v x+b_{3} y=0 .
$$

This is a 3 -sheeted cover of $B_{3}$ inside $Y_{4}$ that is singular at $z=0$ where the sheets come together. The sheets are separated when the singularity is resolved and the resulting divisor, $\tilde{\mathcal{C}}_{F}$, is smooth inside $\tilde{Y}_{4}$. The usefulness of $\mathcal{C}_{F}$, at least initially, is that it provides a very efficient probe of the resolved cycles in $\tilde{Y}_{4}$. Given a (possibly singular) holomorphic surface $\mathcal{G}$ inside $\mathcal{C}_{F}$ before the resolution, we know exactly what the intersections of its proper transform $\tilde{\mathcal{G}}$ will be with the surfaces of $\tilde{Y}_{4}$ that degenerate in the blow-down limit $\tilde{Y}_{4} \rightarrow Y_{4}$. We can indirectly specify a $G$-flux in $\tilde{Y}_{4}$, then, by specifying $\mathcal{G}$ in $Y_{4}$; this allows many chirality computations to be performed without having to work with the resolved geometry explicitly. One must take care, however, that $\mathcal{G}$ is quantized properly in light of the flux quantization rule [70]

$$
G_{4}+\frac{1}{2} c_{2}\left(\tilde{Y}_{4}\right) \in H^{2,2}\left(\tilde{Y}_{4}, \mathbb{Z}\right)
$$

In particular, $c_{2}\left(\tilde{Y}_{4}\right)$ is not an even class in our $\tilde{Y}_{4}$ so $\mathcal{G}$ must be a non-integral surface class of the right type.

Often we are interested in going beyond chirality computations and for this we need a more careful specification of $G$-flux and, indeed, $C_{3}$. For that, we would like to start not with the homology class of a holomorphic surface $11 \mathcal{G}$ in $\mathcal{C}_{F}$ but instead a line bundle $\mathcal{N}_{F}$ on $\tilde{\mathcal{C}}_{F}$ (or the corresponding sheaf on $\mathcal{C}_{F}$ ). If we let $\iota_{F}$ denote the embedding map

$$
\iota_{F}: \tilde{\mathcal{C}}_{F} \rightarrow \tilde{Y}_{4}
$$

and $A_{F}$ the connection of $\mathcal{N}_{F}$, we can obtain a 3 -form from the push-forward $\iota_{F *} A_{F}$. This can't be our final $C_{3}$ because the resulting $G$-flux would be an integral $(2,2)$-form that will not satisfy (3.5) for the resolution $\tilde{Y}_{4}$ of our $Y_{4}(3.2)$. We can consider, however, the ramification divisor $r_{F}$ of the covering

$$
p_{F}: \tilde{\mathcal{C}}_{F} \rightarrow B_{2}
$$

and the bundle $\mathcal{O}_{\tilde{\mathcal{C}}_{F}}\left(r_{F}\right)$. As we show in Appendix $\mathrm{A}$, we should consider not $\mathcal{O}_{\tilde{\mathcal{C}}_{F}}\left(r_{F}\right)$ but rather a simple twist of this bundle that we denote by $\mathcal{L}_{\hat{r}}$ A.86). This bundle will have a connection $A_{\hat{r}}$ from which we can obtain a properly quantized $G$-flux as

$$
C_{3}=\iota_{F *}\left(A_{F}-\frac{1}{2} A_{\hat{r}}\right)+\frac{1}{2} C_{3}^{(0)} \leftrightarrow G_{4} \sim \iota_{F *}\left(c_{1}\left(\mathcal{N}_{F}\right)-\frac{1}{2} c_{1}\left(\mathcal{L}_{\hat{r}}\right)\right)+\frac{1}{2} G_{4}^{(0)}
$$

Here, $G_{4}^{(0)}$ is a Cartan flux that corresponds to a particular worldvolume gauge field background (A.108) and $C_{3}^{(0)}$ is a corresponding potential. As discussed in Appendix A. it is often necessary to add further integral worldvolume $G$-fluxes to (3.8) to preserve the $E_{6}$ gauge group.

transform of (3.4) inside the resolution is actually reducible and the spectral divisor corresponds to a particular component. To avoid this difficulty, we can alternatively define $\mathcal{C}_{F}$ via the equation $y^{2}=x^{3}+f x z^{4} v^{4}+g z^{6} v^{6}$. The proper transform of this is irreducible and corresponds precisely to the spectral divisor. This latter equation is therefore easier to work with though it obscures the dependence of $\mathcal{C}_{F}$ on the moduli $b_{m}$.

${ }^{11}$ Which happens to be a divisor in $\mathcal{C}_{F}$, since $\mathcal{C}_{F}$ is a threefold. 
For $C_{3}$ specified as in (3.8), the spectral divisor describes precisely how the flux data impacts the $E_{6}$ gauge theory on $\mathbb{R}^{3,1} \times B_{2}$. Recall that the holomorphic data of the gauge theory consists of a Higgs bundle spectral cover, $\mathcal{C}_{\text {loc }}$ and a line bundle $\mathcal{N}_{\text {loc }}$ on $\mathcal{C}_{\text {loc }}$. More specifically, $\mathcal{C}_{\text {loc }}$ is a 3 -sheeted cover of $B_{2}$ that lives in the total space of $K_{B_{2}}$ or some suitable compactification thereof. Introducing the projection map

$$
p_{l o c}: \mathcal{C}_{l o c} \rightarrow B_{2}
$$

the data $\left(\mathcal{C}_{\text {loc }}, \mathcal{N}_{\text {loc }}\right)$ determine the configuration of an adjoint scalar $\Phi_{l o c}$ and adjoint gauge bundle $V_{l o c}$ of the gauge theory on $B_{2}$ as [71]

$$
\Phi_{l o c} \sim p_{\text {loc* }} s \quad V_{\text {loc }} \sim p_{\text {loc* }} \mathcal{N}_{\text {loc }}
$$

where $s$ is a normal coordinate to $B_{2}$ in the ambient 3 -fold.

The Higgs bundle spectral cover emerges from $\tilde{Y}_{4}$ as the restriction of $\tilde{\mathcal{C}}_{F}$ to (the proper transform of) $\pi^{*} B_{2}$

$$
\mathcal{C}_{\text {loc }}=\left.\tilde{\mathcal{C}}_{F}\right|_{\pi^{*} B_{2}}
$$

while the bundle $\mathcal{N}_{\text {loc }}$ is just the restriction of $\mathcal{N}_{F}$

$$
\mathcal{N}_{\text {loc }}=\left.\mathcal{N}_{F}\right|_{\mathcal{C}_{l o c}}
$$

To preserve the $E_{6}$ gauge group, $\mathcal{N}_{\text {loc }}$ must satisfy $c_{1}\left(p_{\text {loc* }} \mathcal{N}_{\text {loc }}\right)=0$. One can make a decomposition of $c_{1}\left(\mathcal{N}_{l o c}\right)$ that is similar to (2.10)

$$
c_{1}\left(\mathcal{N}_{l o c}\right)=\lambda \gamma_{l o c}+\frac{r_{l o c}}{2}
$$

The bundle $\mathcal{L}_{\hat{r}}$ that we obtain by twisting $\mathcal{O}_{\mathcal{C}_{F}}\left(r_{F}\right)$ in (A.86) is constructed so that

$$
\left.\mathcal{L}_{\hat{r}}\right|_{\mathcal{C}_{l o c}}=\mathcal{O}_{\mathcal{C}_{l o c}}\left(r_{l o c}\right)
$$

which means the divisor $\gamma_{l o c}$ from (3.13) is identified with the holomorphic surface $\mathcal{G}$ that is Poincare dual to the $G$-flux in (3.8) (up to Cartan corrections)

$$
\gamma_{l o c}=\left.\mathcal{G}\right|_{\mathcal{C}_{l o c}} .
$$

\subsubsection{A Comment on the Compact Realization of $\mathcal{C}_{l o c}$ as $\left.\mathcal{C}_{F}\right|_{\pi^{*} B_{2}}$}

Before proceeding, let us note that we provide a complete description of the surface $\mathcal{C}_{\text {loc }}$ in Appendix $\mathrm{A}$. There, we demonstrate that $\mathcal{C}_{\text {loc }}$ can be viewed as a complete intersection inside an $\mathbb{F}_{1}$-fibration over $B_{2}$ that we denote by $E_{4} 12$. Letting $[u, q]$ denote coordinates

\footnotetext{
${ }^{12}$ We resolve $Y_{4}$ by performing a series of blow-ups in the ambient space $W_{5}$ and passing to the proper transform. Above generic points on $B_{2}$, all three sheets of $\tilde{\mathcal{C}}_{F}$ in $\tilde{Y}_{4}$ meet the same node, say $C$, of the singular fiber. The divisor obtained by fibering $C$ over $B_{2}$ is the restriction to $\tilde{Y}_{4}$ of an exceptional divisor (denoted $E_{4}$ in Appendix $\mathrm{A}$ ) of the blown-up ambient space. The $\mathbb{F}_{1}$-fibration over $B_{2}$ is this exceptional divisor and the two defining equations are the defining equations of $\mathcal{C}_{F}$ as a complete intersection in the ambient 5-fold.
} 
on the fiber and $[W, X]$ coordinates on the base, the $\mathbb{F}_{1}$-fibration is specified by promoting $u, q, W, X$ to sections of the indicated bundles

\begin{tabular}{c|c} 
Section & Bundle on $E_{4}$ \\
\hline$W$ & $f$ \\
$X$ & $f+2 c_{1, B_{2}}$ \\
$u$ & $b$ \\
$q$ & $b+f+3 c_{1, B_{2} .}$.
\end{tabular}

The defining equations of $\mathcal{C}_{\text {loc }}$ inside $E_{4}$ are

$$
\begin{aligned}
W q^{2} & =u^{2}\left(X^{3}+W^{2} X f+W^{3} g\right) \\
0 & =b_{3} q+b_{2} u X+b_{0} u W .
\end{aligned}
$$

Above generic points in $B_{2}$, the first equation defines an anti-canonical curve in the $\mathbb{F}_{1}$ fiber, which is just an elliptic curve. Closer inspection tells us, in fact, that this equation specifies an elliptically fibered Calabi-Yau 3-fold $Z_{3, F}$ with base $B_{2}$ and section. As we review in section 4.1. this is isomorphic to the heterotic 3 -fold when a heterotic dual exists. We stress, however, that the appearance of this (auxiliary) Calabi-Yau 3-fold does not depend on the existence of a heterotic dual. We can map its realization to the more conventional one as a hypersurface in a $\mathbb{P}_{1,2,3}^{2}$ bundle over $B_{2}$ by implicitly defining new coordinates $v, x, y$ through

$$
\begin{aligned}
W & \equiv v^{2} \\
X & \equiv x \\
q & \equiv y \\
u & \equiv v .
\end{aligned}
$$

This is the Higgs bundle spectral cover of the $E_{6}$ gauge theory on $\mathbb{R}^{3,1} \times B_{2}$. It has been known for a while that the non-compact Higgs bundle spectral cover, which can be viewed as a non-compact hypersurface in the total space of $K_{B_{2}}$, emerges from $\left.\tilde{\mathcal{C}}_{E}\right|_{\pi^{*}} B_{2}$ [30, 32] but the full compact surface that appears hasn't been investigated before 13 .

Though the equations (3.17) appear in the explicit $E_{6}$ resolution of appendix A, this compact realization of $\mathcal{C}_{l o c}$ begins to exist once $E_{4}$ begins to exist. In particular, it applies to the cases of $S U(5)$ and $S O(10)$ gauge symmetry. Utilizing (3.18), the appropriate generalization of (3.17) to write the defining equation for $\mathcal{C}_{l o c}$ inside $Z_{3, F}$ is

$$
f_{\mathcal{C}_{\text {loc }}}=\sum_{q=1}^{n} b_{q} v^{n-q} x^{n_{x}} y^{n_{y}}
$$

where $q=2 n_{x}+3 n_{y}$ and $G=E_{6}, S O(10), S U(5)$ correspond to $n=3,4,5$. Recall that the flux parameter $\lambda$ is half-integral for $n$ odd and integral for $n$ even.

\footnotetext{
${ }^{13}$ For most computations in the $E_{6}$ gauge theory, the compact surface will not matter as the non-compact spectral cover contains all the relevant information. To describe instanton vanishing loci, however, details of how the non-compact spectral cover is compactified become very important. Most local model studies use a compactification that sits in $\mathbb{P}\left(\mathcal{O} \oplus K_{B_{2}}\right)$ but the actual surface that appears in $\tilde{Y}_{4}$ is not isomorphic to this. It is, however, isomorphic to the heterotic spectral cover when a heterotic dual exists.
} 
That the fourth exceptional divisor $E_{4}$ plays a special role in obtaining a nice compactification of an $S U(n)$ spectral cover is not surprising: if it sat in the third exceptional divisor $E_{3}$, then an $S U(4)$ gauge symmetry would admit a spectral cover description, which it does not. If, on the other hand, $\mathcal{C}_{\text {loc }}$ sat in the fifth exceptional divisor $E_{5}$ then this compactification of the spectral cover would describe cases of $S O(10)$ gauge symmetry, but not $S U(5)$. Simply put, this compactification of the spectral cover begins to exist when the spectral cover description of the gauge theory begins to exist.

\subsection{M5 Instantons}

We now review some properties of M5 instantons and the conditions under which they to generate superpotential couplings. For this, we consider Euclidean M5's wrapping vertical divisors of $Y_{4}$, that is divisors of the form $\pi^{*} D 3$ for surfaces $D 3$ in $B_{3}$. In the IIB limit, these descend to D3-instantons while they correspond to heterotic worldsheet instantons when a heterotic dual exists. For the purpose of this paper, we are interested in two types of fields on the M5: worldvolume fermions and the chiral 2-form $\phi_{2}$. The former play a similar role to the right-moving fermions on the heterotic worldsheet in that our only concern is whether the zero mode structure is appropriate for a superpotential coupling. The 2-form, on the other hand, is trickier to study but yields a partition function that captures the instanton dependence on $C_{3}$ flux and the complex structure moduli. We address each of these fields in turn.

\subsubsection{Fermi Zero Modes}

The spectrum of Fermi zero modes on an M5 instanton is well-known (see for instance [49])

$$
2 \times\left[h^{0,0}(M 5)+h^{0,1}(M 5)+h^{0,2}(M 5)+h^{0,3}(M 5)\right] .
$$

In the absence of fluxes which lift some of the Fermi zero modes, the instanton must give rise to the 2 'universal' modes that arise from $h^{0,0}(M 5)=1$ in order to generate a superpotential correction. In particular, we must have

$$
h^{0,1}(M 5)=h^{0,2}(M 5)=h^{0,3}(M 5)=0 .
$$

Note that Witten's arithmetic genus condition $\chi\left(M 5, \mathcal{O}_{M 5}\right)=1$ [10] is a necessary but not sufficient condition for this.

The M5-instantons we study have a special structure. They are 'vertical' M5instantons which are elliptic fibrations over a base surface that we are denoting by D3

$$
P: M 5 \rightarrow D 3 .
$$

It is often helpful to rephrase (3.21) in terms of cohomologies on D3 which, as the notation suggests, we think of as the surface wrapped by a D3-instanton in the IIB picture. We do this with the Leray spectral sequence that starts with

$$
E_{2}^{i j}=H^{i}\left(S, R^{j} P_{*} \mathcal{O}_{M 5}\right)
$$

and terminates on the second page [54]. Using explicit results for the direct images $R^{j} P_{*} \mathcal{O}_{M 5}$

$$
R^{0} P_{*} \mathcal{O}_{M 5}=\mathcal{O}_{D 3} \quad R^{1} P_{*} \mathcal{O}_{M 5}=N_{D 3 / M 5}=K_{D 3} \otimes N_{D 3 / B_{3}}^{-1}
$$


we can rewrite $h^{p}\left(M 5, \mathcal{O}_{M 5}\right)$ for $p=1,2,3$ as

$$
\begin{aligned}
h^{1}\left(M 5, \mathcal{O}_{M 5}\right) & =h^{0}\left(D 3, K_{D 3} \otimes N_{D 3 / B_{3}}^{-1}\right)+h^{1}\left(D 3, \mathcal{O}_{D 3}\right) \\
& =h^{2}\left(D 3, N_{D 3 / B_{3}}\right)+h^{1}\left(D 3, \mathcal{O}_{D 3}\right) \\
h^{2}\left(M 5, \mathcal{O}_{M 5}\right) & =h^{2}\left(D 3, K_{D 3} \otimes N_{D 3 / B_{3}}^{-1}\right)+h^{2}\left(D 3, \mathcal{O}_{D 3}\right) \\
& =h^{1}\left(D 3, N_{D 3 / B_{3}}\right)+h^{2}\left(D 3, \mathcal{O}_{D 3}\right) \\
h^{3}\left(M 5, \mathcal{O}_{M 5}\right) & =h^{2}\left(D 3, K_{D 3} \otimes N_{D 3 / B_{3}}^{-1}\right) \\
& =h^{0}\left(D 3, N_{D 3 / B_{3}}\right) .
\end{aligned}
$$

To avoid fermi zero modes, then, the divisor $D 3$ in $B_{3}$ must be rigid in the sense that

$$
h^{1}\left(D 3, \mathcal{O}_{D 3}\right)=h^{2}\left(D 3, \mathcal{O}_{D 3}\right)=0
$$

and further the normal bundle of $D 3$ inside the base $B_{3}$ of $Y_{4}$ must have no cohomology

$$
h^{m}\left(D 3, N_{D 3 / B_{3}}\right)=0 \quad m=0,1,2
$$

For the first condition to hold, $D 3$ should be a $d P_{n}$ or $\mathbb{F}_{m}$ surface. It is sometimes possible that Fermi zero modes can be lifted in the presence of a nontrivial $G$-flux through a coupling of the form $\theta(\Gamma G) \theta[72-75]$. One can think of such a $G$ as inducing a map $G: \Omega^{0,2}(M 5) \rightarrow \Omega^{2,0}(M 5)$ whose kernel corresponds to the unlifted zero modes. We will restrict ourselves to instantons with $h^{0,2}(M 5)=0$ in this paper, and so will not appeal to such a mechanism.

\subsubsection{Chiral 2-form}

We now turn to the partition function of the chiral 2-form field on the M5-brane worldvolume. We call this partition function $\mathcal{Z}_{\phi}$. Since no covariant Lagrangian formulation is known, it can be tricky to compute this quantity directly. Following Witten [66], however, we can make significant progress by looking at the transformation properties of $\mathcal{Z}_{\phi}$ under bulk gauge transformations. In general, the partition function of our M5 instanton will be proportional to

$$
e^{i \tau_{M 5} \int_{M 5} C_{6}} \mathcal{Z}_{\phi}
$$

where $C_{6}$ is the 6 -form potential dual to the $M$-theory 3 -form, $C_{3}$. Just as with (2.18), the product $e^{i \tau_{M 5} \int_{M 5} C_{6}} \mathcal{Z}_{\phi}$ is invariant under bulk gauge transformations $C_{3} \rightarrow C_{3}+d \Lambda_{2}$ but the individual terms in the product are not. Rather, $C_{6}$ transforms as

$$
C_{6} \rightarrow C_{6}+\frac{1}{2} \Lambda_{2} \wedge G_{4}
$$

and the 2 -form partition function $\mathcal{Z}_{\phi}$ transforms in the opposite way due to the coupling

$$
S_{M 5} \sim \int_{M 5}\left(-\frac{1}{2} \phi_{2} \wedge G_{4}+\ldots\right)
$$

and the transformation law of $\phi_{2}$

$$
\phi_{2} \rightarrow \phi_{2}+\Lambda_{2}
$$


Because of this, the partition function $\mathcal{Z}_{\phi}$ is not a function at all; it is actually a holomorphic section of a line bundle on the moduli space of $C_{3}$ 's. The coupling (3.30) induces a source for $\phi_{2}$ so the restriction of $G_{4}$ to the instanton must be 14 cohomologically zero for $\mathcal{Z}_{\phi}$ to have any hope of being nonzero

$$
\left.G_{4}\right|_{M 5}=0 \text { in } H^{2,2}(M 5) .
$$

Correspondingly, the moduli space of allowed $C_{3}$ 's consists of harmonic 3 -forms modulo large gauge transformations. This is the intermediate Jacobian of the M5

$$
\mathcal{J}(M 5)=H^{3}(M 5, \mathbb{R}) / H^{3}(M 5, \mathbb{Z})
$$

which is an Abelian variety. The transformation law $\mathcal{Z}_{\phi} \rightarrow \mathcal{Z}_{\phi} e^{-i \int_{M 5} \frac{1}{2} \phi_{2} \wedge G_{4}}$ implies that $\mathcal{Z}_{\phi}$ is a holomorphic section of a line bundle on $\mathcal{J}(M 5)$ whose first Chern class is a principal polarization. This is almost enough to fix the $C_{3}$-dependence of $\mathcal{Z}_{\phi}$ because it means that $\mathcal{Z}_{\phi}$ is proportional to a $\theta$ function on $\mathcal{J}(M 5)$. However, there is a $\theta$ function for each principal polarization, and it remains to determine which theta function is the 'right' one. Witten introduced a general procedure for determining this for given spin structures on $M 5$ and $\tilde{Y}_{4}$ [66] but applying it in practice is quite subtle15. If the right $\theta$ function can be found, however, we can in principle study the dependence of $\mathcal{Z}_{\phi}$ on the complex structure moduli of $Y_{4}$ (or at least their restrictions to $M 5$ ) by using the fact that $\mathcal{J}(M 5)$ varies holomorphically in holomorphic families 16 .

Though straightforward in principle, a careful study of $\mathcal{Z}_{\phi}$ along these lines is difficult in practice. We believe it is helpful to keep in mind the analogy to heterotic worldsheet instantons, where the problem of 'choosing the right $\theta$ function' also arises. There, we encountered the Pfaffian of a Dirac operator over fermions on $c_{\text {Het }}$ coupled to a degree zero line bundle $\mathcal{L}_{\Gamma}$. As with $\mathcal{Z}_{\phi}$, the anomalous gauge transformation of $\operatorname{Pfaff}\left(c_{H e t}, D_{-, \mathcal{L}_{\Gamma}}\right)$ implied that it was proportional to a $\theta$ function on an Abelian variety, the Jacobian $\mathcal{J}_{0}\left(c_{\mathrm{Het}}\right)$. Determining the 'right' $\theta$ function could have been tricky but we were aided by the fact that we knew the exact vanishing locus of $\operatorname{Pfaff}\left(c_{H e t}, D_{-, \mathcal{L}_{\Gamma}}\right)$. This is because we could identify the relevant Fermi zero modes, which cause the prefactor to vanish, in a way that didn't depend on a choice of spin structure on $c_{\text {Het }}$. This extra information of the vanishing locus fixed $\operatorname{Pfaff}\left(c_{H e t}, D_{-, \mathcal{L}_{\Gamma}}\right)$ completely and provided a prescription for determining the proper $\theta$ function to associate with each choice of spin structure.

Pursuing a similar strategy for M5's is difficult because we do not a priori know the precise vanishing locus in general. Unlike the case of heterotic worldsheet instantons, the vanishing is not due to Fermi zero modes but rather to the physics of the chiral 2-form. Nevertheless, we might hope to gain some intuition from physics and, in particular, our expectations from the IIB limit. Even though it is not directly attributable to Fermi zero modes, we expect the vanishing structure of $\mathcal{Z}_{\phi}$ to be captured by the physics of 3-7 zero modes in IIB that arise from open strings with one end on the D3 and the other

\footnotetext{
${ }^{14}$ In the absence of M2-brane insertions. See section 4.2 for a brief discussion.

${ }^{15}$ This problem has received further attention in recent work related to D3-brane instantons in orientifold models and their F-theory lifts [55].

${ }^{16}$ This follows because the condition $h^{3,0}(M 5)=0$ allows us to define a complex structure on $\mathcal{J}(M 5)$ without ambiguity (i.e. there is no distinction between the Griffiths and Weil Jacobians).
} 
end on a stack of 7-branes. This physics, in turn, should be controlled by cohomologies that can be interpreted in terms of fermions localized near $B_{2}$ in $Y_{4}$.

As we will discuss in section 5.1, this is exactly what happens when a heterotic dual exists! Even though heterotic computations involve line bundle cohomologies on curves 'away from the $E_{6}$ singularity' in the usual sense, they can be reformulated isomorphically in the local geometry near the singularity. This reformulation does not explicitly involve the structures necessitated by the heterotic dual in any way and suggests that the physics behind the vanishing is effectively captured by the local geometry near $B_{2}$, as we would expect from a 3-7 interpretation. This leads to a natural conjecture for the vanishing locus of certain M5 partition functions in F-theory compactifications without heterotic duals that is simple and allows for straightforward computation.

\section{Heterotic/F-theory Duality}

Having reviewed both worldsheet instantons in the heterotic string and M5-instantons in F-theory, let us now discuss how these effects are related via duality. We will begin by discussing aspects of heterotic/F-theory duality, including the relation of the 'cylinder map' to the spectral divisor formalism. By intersecting with the M5-brane divisor, we then restrict this story to the 'miniature' version of the cylinder map that relates heterotic worldsheet instantons to M5 instantons in F-theory duals. We use the compact realization of the local model spectral cover inside the F-theory compactification to rephrase the vanishing locus in terms of cohomology computations in the local geometry near the $E_{6}$ singularity. In this way, we motivate a conjecture for the vanishing locus of the M5 instanton partition function in a class of F-theory compactifications that do not admit heterotic duals.

\subsection{Heterotic/F-theory Duality}

To construct models with a heterotic dual, we specialize the 4-fold (3.2) by taking the base 3 -fold $B_{3}$ to be a $\mathbb{P}^{1}$-fibration over a surface $B_{2}$ specified by a line bundle $N$

$$
B_{3}=\mathbb{P}(\mathcal{O} \oplus N)
$$

We introduce the notation $s$ for the divisor class of the section of $B_{3}$ corresponding to the ' $\mathcal{O}$ ' factor and let $[Z, W]$ denote homogeneous coordinates on the fiber

\begin{tabular}{c|c} 
Section & Bundle \\
\hline$Z$ & $\mathcal{O}(s)$ \\
$W$ & $\mathcal{O}(s+t)$
\end{tabular}

where $t$ is a divisor on $B_{2}$ and, as usual, we do not notationally distinguish between divisors on $B_{2}$ and their pullbacks to $B_{3}$. With this notation, the divisor we called $B_{2}$ in section 3 is referred to as $s$ in order to distinguish it from the other section $s+t$ of $B_{3}$, which is also isomorphic to $B_{2}$.

The 4 -fold $Y_{4}$ that results from (3.2) and this special choice of $B_{3}$ is a K3-fibration over $B_{2}$ and admits a heterotic dual through fiberwise application of the standard equivalence of F-theory on $\mathrm{K} 3$ and $E_{8} \times E_{8}$ heterotic strings on $T^{2}$. We recover the 
heterotic data by taking the stable degeneration limit, wherein $Y_{4}$ splits into a pair of $d P_{9}$-fibrations glued together along the elliptically fibered Calabi-Yau 3-fold $Z_{H e t}$. Each $d P_{9}$ fibration, along with the corresponding $G$-flux, determines one of the two heterotic bundles. As we focus attention on just one $E_{8}$ factor in this paper, namely one that is broken to $E_{6}$ by the $S U(3)$ bundle $V$, we restrict our attention to the $d P_{9}$ fibration that contains the surface of $E_{6}$ singularities. This 4 -fold, which we denote by $Y_{4}^{\prime}$, can be described as a hypersurface in a $\mathbb{P}_{1,2,3}^{2}$-bundle, $W_{5}^{\prime}$, defined by the equation

$$
y^{2}=x^{3}+f x Z^{4} v^{4}+g Z^{6} v^{6}+Z^{2} W v^{3}\left[a_{0} Z^{3} v^{3}+a_{2} Z v x+a_{3} y\right]
$$

where the objects that appear are sections of the indicated bundles

\begin{tabular}{c|c} 
Section & Bundle \\
\hline$v$ & $\mathcal{O}(\sigma)$ \\
$x$ & $\mathcal{O}\left(2\left[\sigma+s+c_{1, B_{2}}\right]\right)$ \\
$y$ & $\mathcal{O}\left(3\left[\sigma+s+c_{1, B_{2}}\right]\right)$ \\
$f$ & $\mathcal{O}\left(4 c_{1, B_{2}}\right)$ \\
$g$ & $\mathcal{O}\left(6 c_{1, B_{2}}\right)$ \\
$a_{0}$ & $\mathcal{O}\left(6 c_{1, B_{2}}-t\right)=\mathcal{O}(\eta)$ \\
$a_{2}$ & $\mathcal{O}\left(4 c_{1, B_{2}}-t\right)=\mathcal{O}\left(\eta-2 c_{1, B_{2}}\right)$ \\
$a_{3}$ & $\mathcal{O}\left(3 c_{1, B_{2}}-t\right)=\mathcal{O}\left(\eta-3 c_{1, B_{2}}\right)$ \\
$Z$ & $\mathcal{O}(s)$ \\
$W$ & $\mathcal{O}(s+t)$.
\end{tabular}

The sections $a_{m}$ are related to the $b_{m}$ of (3.2) by restriction to $B_{2}$

$$
a_{m}=\left.b_{m}\right|_{B_{2}}
$$

and hence reflect the 'leading' behavior of the $b_{m}$ 's near the surface of $E_{6}$ singularities at $Z=0$.

The divisor $W=0$ is nothing other than the heterotic Calabi-Yau $Z_{\text {Het }}(2.2)$. To describe the heterotic bundle, let us follow the spectral divisor $\mathcal{C}_{F}$ (3.4) through the stable degeneration limit to obtain the 3 -fold given by 17

$$
a_{0} Z^{3} v^{3}+a_{2} Z v x+a_{3} y=0
$$

This 3 -fold is often referred to as the 'cylinder', which we denote by $\mathcal{C}_{\text {cyl }}$, and it is a 3 -sheeted cover of $B_{3}$ inside $Y_{4}^{\prime}$ that is singular where the sheets come together at $Z=0$. The 3 -fold $\mathcal{C}_{\text {cyl }}$ is a natural object for capturing the behavior of our $d P_{9}$ fibration that arises as follow: 18 .

\footnotetext{
${ }^{17}$ Recall that here, in the case of heterotic F-theory duality, we used $Z=0$ rather than $z=0$ for the GUT stack in $B_{3}$.

18 If one prefers, the equation $y^{2}=x^{3}+f x Z^{4} v^{4}+g Z^{6} v^{6}$ less the components that coincide with the section can be used to followed $\mathcal{C}_{F}$ through the stable degeneration limit. Though the spectral divisor was irreducible, this 3 -fold is not; it has acquired an extra component at $W=0$ corresponding to $Z_{H e t}$ itself. If we discard this component, what remains is a 3 -fold that can be defined in $W_{5}^{\prime}$ as
}

$$
\begin{aligned}
\mathcal{C}_{c y l}: \quad y^{2} & =x^{3}+f x Z^{4} v^{4}+g Z^{6} v^{6} \\
0 & =a_{0} Z^{3} v^{3}+a_{2} Z v x+a_{3} y
\end{aligned}
$$


Our presentation of $Y_{4}^{\prime}$ distinguishes two curve classes of each $d P_{9}$ fiber: the elliptic fiber $x_{9}$ of $d P_{9}$ and the base, $e_{9}$. The remaining curves in $d P_{9}$ or, more specifically, the classes in $H_{2}\left(d P_{9}, \mathbb{Z}\right)$ that are orthogonal to $x_{9}$ and $e_{9}$, will be permuted as we move along various paths in $B_{2}$. The lattice of curve classes $C$ in $d P_{9}$ that satisfy $C \cdot x_{9}=C \cdot e_{9}=0$ is isomorphic to $H_{2}\left(d P_{8}, \mathbb{Z}\right)$ which, itself, is a copy of the $E_{8}$ root lattice. As a result, a generic $d P_{9}$ fibration will interchange all $240 E_{8}$ roots (that is all curve class $C \in H_{2}\left(d P_{9}, \mathbb{Z}\right)$ with $C \cdot x_{9}=C \cdot e_{9}=0$ and $\left.C^{2}=-2\right)$ with one another according to actions of the Weyl group of $E_{8}$. Of the 240 roots of $E_{8}$, though, 72 are distinguished in $Y_{4}^{\prime}$ as the roots of $E_{6}$. This means that the Weyl group of the $S U(3)$ commutant of $E_{6}$ inside $E_{8}$ controls the fibration in the case we consider, there the $d P_{9}$ fibration is non-generic so as to give a surface of $E_{6}$ singularities at $Z=0$. The action of the Weyl group is governed by the decomposition of $E_{8}$ roots under $E_{8} \rightarrow E_{6} \times S U(3)$

$$
240 \rightarrow(72,1)+(1,6)+[(27,3)+\mathrm{cc}]
$$

The complete Weyl group action can therefore be determined by following a trio of roots that share a common $\mathbf{2 7}$ weight and mix under the action of $S U(3)$. Given any such root, $C$, we can construct an exceptional line $\ell=x_{9}+e_{9}-C$ that is orthogonal to $e_{9}$ but meets $x_{9}$ in exactly one point. The cylinder $\mathcal{C}_{\text {cyl }}$ is the sum of three exceptional lines in the highest weight state $(1,0,0,0,0,0)$ of the $\mathbf{2 7}$ that mix as a triplet under $S U(3)$. Each exceptional line meets $x_{9}$ exactly once and a distinguished node of the $E_{6}$ singular fiber exactly once.

From this general description, we expect $\left.\mathcal{C}_{\text {cyl }}\right|_{W=0}$ to comprise a set of 3 points on the elliptic fiber above each generic point in the base $B_{2}$. This is a 3 -sheeted cover of $B_{2}$ inside $Z_{H e t}$ that is nothing other than the heterotic spectral cover $\mathcal{C}_{\text {Het }}$ :

$$
\left.\mathcal{C}_{\text {cyl }}\right|_{Z_{\text {Het }}}=\mathcal{C}_{H e t}
$$

Restricting to $Z_{\text {Het }}=\{W=0\}$ sets $Z=1$ in (4.6) and recovers the well-known equation for an $S U(3)$ spectral cover in the heterotic string. Likewise, we can check that $\mathcal{C}_{\text {cyl }}$ is in fact a $\mathbb{P}^{1}$-fibration over $\mathcal{C}_{H e t}$ though this $\mathbb{P}^{1}$ fibration is singular in $Y_{4}^{\prime}$ where the sheets come together. Nevertheless, we can define a projection

$$
p_{\text {cyl }}: \mathcal{C}_{\text {cyl }} \rightarrow \mathcal{C}_{\text {Het }}
$$

and use this to describe the 'cylinder' map relating heterotic bundle data to F-theory $G$-flux. The idea is as follows. Given a bundle $\mathcal{N}_{\text {Het }}$ on $\mathcal{C}_{\text {Het }}$ that completes the heterotic bundle data $\left(\mathcal{C}_{\mathrm{Het}}, \mathcal{N}_{\mathrm{Het}}\right)$, we write (2.10)

$$
c_{1}\left(\mathcal{N}_{H e t}\right)=c_{1}(\mathcal{O}(\lambda \gamma+r / 2))
$$

for a half-integral divisor class $\gamma$ on $\mathcal{C}_{H e t}$. From $\gamma$ we obtain a $G$-flux by pulling $\gamma$ back to $\mathcal{C}_{\text {cyl }}$ and pushing it forward to $Y_{4}^{\prime}$ via the embedding

$$
\iota_{c y l}: \mathcal{C}_{c y l} \rightarrow Y_{4}^{\prime}
$$

as in

$$
G_{4}=\iota_{c y l *} p_{c y l}^{*} \lambda \gamma
$$


where we take the Poincare dual of the resulting holomorphic surface class to obtain the $(2,2)$-form $G_{4}$.

We would like to study this map a little more carefully in a way that doesn't require splitting $c_{1}\left(\mathcal{N}_{\text {Het }}\right)$ into a pair of half-integral divisor classes. This will allow us to work at the level of line bundles and make a more direct connection to $C_{3}$. Let $\mathcal{N}_{c y l}$ be the bundle on $\mathcal{C}_{c y l}$ obtained as the pull-back of $\mathcal{N}_{\text {Het }}$

$$
\mathcal{N}_{\text {cyl }}=p_{\text {cyl }}^{*} \mathcal{N}_{\text {Het }} .
$$

Further, let $A_{c y l}$ be the connection of $\mathcal{N}_{c y l}$ and $A_{r, c y l}$ the connection of the bundle $\mathcal{O}\left(r_{c y l}\right)$ with $r_{c y l}$ the ramification divisor of the covering $p_{c y l}: \mathcal{C}_{c y l} \rightarrow B_{3}$. We take

$$
C_{3}=\iota_{c y l *}\left(A_{c y l}-\frac{1}{2} A_{r, c y l}\right)+\ldots
$$

which leads to

$$
G_{4}=\iota_{c y l *}\left(c_{1}\left(\mathcal{N}_{c y l}\right)-\frac{1}{2} c_{1}\left(\hat{L}_{r}\right)\right)+\ldots
$$

This is completely equivalent to (4.13) because $\left.r_{c y l}\right|_{\mathcal{C}_{H e t}}=r$.

We now turn to the restriction of $\mathcal{C}_{\text {cyl }}$ to the singular fibration over $B_{2}, Z=0$. Because $\mathcal{C}_{\text {cyl }}$ is singular here, we must pass to the resolved geometry $\tilde{Y}_{4}^{\prime}$ to obtain a proper description. There is a lot we can say on general grounds, though, from the discussion of $\mathcal{C}_{\text {cyl }}$ above. Firstly, the three exceptional lines that comprise $\mathcal{C}_{\text {cyl }}$ above generic points in $B_{2}$ intersect the nodes of the $E_{6}$ singular fiber according to the highest weight of the $\mathbf{2 7},(1,0,0,0,0,0)$. This means that $\mathcal{C}_{\text {cyl }}$ meets a distinguished node of the singular fiber in 3 points above any generic point in $B_{2}$ so that $\left.\mathcal{C}_{\text {cyl }}\right|_{Z=0}$ is a 3 -sheeted cover of $B_{2}$. Further, we know that $\mathcal{C}_{\text {cyl }}$ is in fact a $\mathbb{P}^{1}$-fibration over $\mathcal{C}_{\text {Het }}$ so $\left.\mathcal{C}_{\text {cyl }}\right|_{Z=0}$ should be isomorphic to $\mathcal{C}_{\text {Het }}$.

We verify all of these things explicitly by studying the resolution $\tilde{Y}_{4}^{\prime}$ of $Y_{4}^{\prime}$ in Appendix A. Indeed, $\left.\mathcal{C}_{\text {cyl }}\right|_{Z=0}$ is completely equivalent to the restriction of our original spectral divisor, $\mathcal{C}_{F}$, to $Z=0$ that is described in section 3.1.1. It is a surface $\mathcal{C}_{\text {loc }}$

$$
\mathcal{C}_{\text {loc }}=\left.\mathcal{C}_{\text {cyl }}\right|_{Z=0}
$$

that can be realized as a complete intersection in the $\mathbb{F}_{1}$-fibration $E_{4} \rightarrow B_{2}$ specified by (3.16)

\begin{tabular}{c|c} 
Section & Bundle on $E_{4}$ \\
\hline$W$ & $\mathcal{O}(f)$ \\
$X$ & $\mathcal{O}\left(f+2 c_{1, B_{2}}\right)$ \\
$u$ & $\mathcal{O}(b)$ \\
$q$ & $\mathcal{O}\left(b+f+3 c_{1, B_{2}}\right)$
\end{tabular}

The defining equations of $\mathcal{C}_{\text {loc }}$ inside $E_{4}$ are

$$
\begin{aligned}
W q^{2} & =u^{2}\left(X^{3}+W^{2} X f+W^{3} g\right) \\
0 & =b_{3} q+b_{2} u X+b_{0} u W
\end{aligned}
$$

The identification (3.18) establishes that the first equation defines a Calabi-Yau 3-fold isomorphic to $Z_{H e t}$. It is then trivial to see that the second defines a surface that is 
isomorphic to $\mathcal{C}_{\text {Het }}$. In fact, we can use the $\mathbb{P}^{1}$-fibration of $\mathcal{C}_{\text {cyl }}$ to translate the heterotic bundle data from $\mathcal{C}_{H e t}$ to $\mathcal{C}_{\text {loc }}$

$$
\begin{aligned}
\gamma_{l o c} & =\gamma_{H e t} \\
\mathcal{N}_{l o c} & =\mathcal{N}_{\text {Het }} .
\end{aligned}
$$

If we like, we can now describe the $G$-flux without any direct reference to the heterotic side at all. We simply define $\mathcal{N}_{c y l}$ by

$$
\mathcal{N}_{c y l}=p_{c y l, l o c}^{*} \mathcal{N}_{l o c}
$$

where

$$
p_{\text {cyl }, \text { loc }}: \mathcal{C}_{\text {cyl }} \rightarrow \mathcal{C}_{\text {loc }}
$$

The 3-form $C_{3}$ and $G$-flux $G_{4}$ are then given as before by (4.15) (4.16). We can also go the other way. Given a line bundle $\mathcal{N}_{c y l}$ on $\mathcal{C}_{c y l}$, we can restrict it to $\mathcal{C}_{l o c}$ to obtain a line bundle $\mathcal{N}_{l o c}$ that completes the spectral data of the $E_{6}$ gauge theory. This reproduces the 'local/global' map of section 3.1 in this case because the bundle $\mathcal{L}_{\hat{r}}$ (A.86) is simply

$$
\mathcal{L}_{\hat{r}}=p_{\text {cyl }}^{*} \mathcal{O}_{\mathcal{C}_{\text {Het }}}\left(r_{H e t}\right)
$$

when a heterotic dual exists (see Appendix A). This is the motivation for the general prescription of section 3.1, which can be applied in the absence of a heterotic dual.

\subsection{Heterotic WS Instantons and their M5 Counterparts}

We now turn to the relation between heterotic worldsheet instantons and M5 instantons in F-theory. The heterotic instanton of interest wraps a curve $\Sigma$ in the base $B_{2}$ of $Z_{H e t}$. The M5-instanton to which this corresponds is just 19 the $d P_{9}$ fibration over $\Sigma$

$$
M 5=\rho^{*} \Sigma
$$

where $\rho$ is the $d P_{9}$ fibration

$$
\rho: Y_{4}^{\prime} \rightarrow B_{2}
$$

The relation between these instantons is essentially a 'miniature' version of the standard heterotic/F-theory duality. On the F-theory side, we have a $d P_{9}$ fibration $M 5$ which can be viewed as an elliptic fibration over a surface $D 3$ that itself is $\mathbb{P}^{1}$-fibered

$$
\pi_{M 5}: M 5 \rightarrow D 3 \quad \nu: D 3 \rightarrow \Sigma .
$$

We also have a 'cylinder', $c_{c y l}$, obtained as the restriction of $\mathcal{C}_{c y l}$ to $M 5$

$$
c_{c y l}=\left.\mathcal{C}_{c y l}\right|_{M 5}
$$

together with a projection $p_{M 5}$ and embedding $\iota_{M 5}$

$$
p_{M 5}: c_{c y l} \rightarrow D 3 \quad \iota_{M 5}: c_{c y l} \rightarrow M 5 .
$$

\footnotetext{
${ }^{19}$ Technically the M5 is the full $K 3$-fibration over $\Sigma, \pi^{*} \Sigma$. Focusing on one of the $d P_{9}$-fibrations is equivalent to the fact in the heterotic string that we have focused on one of the bundles $V$ responsible for breaking one $E_{8}$ factor, ignoring the bundle $\tilde{V}$ which breaks the other $E_{8}$ factor.
} 
Inside the $M 5$ we have 20 at $W=0$ the elliptic fibration over $\Sigma, \mathcal{E}=\pi_{M 5^{2}}^{*}$

$$
\pi_{M 5, h e t}: \mathcal{E} \rightarrow \Sigma
$$

and the restriction of $c_{c y l}$ to $\mathcal{E}$ is nothing more than the curve $c_{H e t}$

$$
\left.c_{c y l}\right|_{\mathcal{E}}=c_{H e t} .
$$

In fact, $c_{c y l}$ is a $\mathbb{P}^{1}$ fibration over $c_{H e t}$ whose projection $21 p_{M 5, c y l}$

$$
p_{M 5, c y l}: c_{c y l} \rightarrow c_{H e t}
$$

allows us to define a 'cylinder' map relating line bundles on $c_{\text {Het }}$ to configurations for $C_{3}$ on $M 5$. The story is exactly as before; we simply restrict everything to M5. Given a line bundle $\mathcal{N}_{c, H e t}$ on $c_{H e t}$, we obtain a line bundle on $c_{c y l}$

$$
\mathcal{N}_{M 5, c y l}=p_{M 5, c y l}^{*} \mathcal{N}_{c, H e t}
$$

and we can go the other way by restriction. Letting $A_{M 5, \text { cyl }}$ be a connection on $\mathcal{N}_{M 5, \text { cyl }}$ and $A_{\hat{R}}$ a connection on $p_{M 5, c y l}^{*}\left(\left.\mathcal{O}_{\mathcal{C}_{H e t}}\left(r_{H e t}\right)\right|_{\mathcal{E}}\right)=\left.\mathcal{L}_{\hat{r}}\right|_{M 5}$, we obtain a 3 -form on $M 5$

$$
C_{3}=\iota_{M 5, c y l *}\left(A_{M 5, c y l}-\frac{1}{2} A_{\hat{R}}\right)
$$

to which Cartan correction terms (A.108) must also be added in the end. Because $A_{M 5, c y l}$ and $A_{\hat{R}}$ are pulled back from connections $A_{c, H e t}$ and $A_{c, \hat{R}}$ on $c_{H e t}$, we can rewrite $C_{3}$ as

$$
\begin{aligned}
\left.C_{3}\right|_{M 5} & =\iota_{M 5, c y l *} p_{M 5, c y l}^{*}\left(A_{c, h e t}-\frac{1}{2} A_{c, \hat{R}}\right) \\
& =\iota_{M 5, c y l *} p_{M 5, c y l}^{*}\left(A_{\Gamma}\right)
\end{aligned}
$$

where $A_{\Gamma}$ is a connection on the bundle $\mathcal{L}_{\Gamma}$ defined in (2.27). Correspondingly,

$$
\begin{aligned}
\left.G_{4}\right|_{M 5} & =\iota_{M 5, c y l *}\left(\left[c_{1}\left(\mathcal{N}_{M 5, c y l}\right)\right]-\frac{1}{2} R_{c y l}\right) \\
& =\iota_{M 5, c y l *} p_{M 5, c y l}^{*}\left(c_{1}\left(\mathcal{N}_{c, H e t}\right)-\frac{1}{2} R\right) \\
& =\iota_{M 5, c y l *} p_{M 5, c y l}^{*} c_{1}\left(\mathcal{L}_{\Gamma}\right) .
\end{aligned}
$$

We saw before that $\mathcal{L}_{\Gamma}$ had to be a degree zero bundle to have any hope of a nonzero superpotential coupling. Here, we see that this condition is equivalent to the requirement that $\left.G_{4}\right|_{M 5}$ be trivial. This is of course a very familiar condition for M5 instantons; a nontrivial flux $\left.G_{4}\right|_{M 5}$ sources $M 2$ brane charge on the M5 worldvolume so a nontrivial superpotential coupling can only be generated if suitable Wilson surface operators, corresponding to insertions of wrapped $M 2$ brane states, are introduced to cancel it [30,49].

\footnotetext{
${ }^{20}$ In section 5.2 we will define an elliptic surface $\mathcal{E} \equiv \pi_{3, F}^{-1} \Sigma$ and give a curve $c_{l o c}$ in it. The surface $\mathcal{E}$ and curve $c_{H e t}$ in $\mathcal{E}$ we define here are isomorphic to the 'other' $\mathcal{E}$ and its curve $c_{\text {loc }}$ in the case of duality. We abuse notation due to isomorphism.

${ }^{21}$ Note that this is just the restriction of the projection map $p_{c y l}$ to the M5.
} 
As we do not introduce any such operators, $\left.G_{4}\right|_{M 5} \neq 0$ forces our instanton corrections to vanish.

We can summarize the basic elements of the mapping as follows

\begin{tabular}{cccc} 
Object on F-theory Side & & Object on Het Side \\
\hline$M 5$ & $\leftrightarrow$ & $\mathcal{E}$ \\
$c_{c y l}$ & $\leftrightarrow$ & $c_{H e t}$ \\
$\mathcal{N}_{c y l}$ & $\leftrightarrow$ & $\mathcal{N}_{c, H e t}$ \\
$C_{3}$ & $\leftrightarrow$ & $\mathcal{L}_{\Gamma}$ \\
$G_{4}$ & $\leftrightarrow$ & $c_{1}\left(\mathcal{L}_{\Gamma}\right)$.
\end{tabular}

In the first three lines, the objects are related through pullback or projection by the $\mathbb{P}^{1}$ fibration $\nu: D 3 \rightarrow \Sigma$ of $D 3$ or the corresponding $\mathbb{P}^{1}$ fibration $p_{M 5, c y l}: c_{c y l} \rightarrow c_{H e t}$ of the restricted cylinder $c_{c y l}$. The last two lines emphasize something nice that we find upon restriction to $M 5$ that does not happen in the $d P_{9}$ fibration, $Y_{4}^{\prime}$. To establish the heterotic/F-theory dictionary between heterotic bundle data and F-theory flux data, we had to split the heterotic bundle according to

$$
c_{1}\left(\mathcal{N}_{H e t}\right)=c_{1}\left(\mathcal{O}\left(\lambda \gamma_{H e t}+r_{H e t} / 2\right)\right)
$$

where $\lambda \gamma_{H e t}$ and $r_{H e t} / 2$ are both half-integral divisor classes on $\mathcal{C}_{H e t}$ in general. The splitting was important not just for convenience but because it is $\lambda \gamma_{H e t}$, not $c_{1}\left(\mathcal{N}_{\text {Het }}\right)$, that maps to $G$-flux 22 . The half-integrality of $\lambda \gamma_{H e t}$ can be cumbersome to deal with, though, as it tempts us to work at the level of Chern classes, where the split into $\lambda \gamma_{\text {Het }}$ and $r_{\text {Het }} / 2$ makes sense, as opposed to the level of line bundles where it doesn't. Working with Chern classes has the potential to lose important information.

When we restrict the heterotic/F-theory duality map to $M 5$, we find a more pleasing situation. The restriction of $\lambda \gamma_{H e t}+r_{H e t} / 2$ is $\lambda \Gamma_{H e t}+R_{H e t} / 2$ where $R_{H e t}$ is the ramification divisor of the covering $p_{c, H e t}: c_{\text {Het }} \rightarrow \Sigma$. The curve $c_{\text {Het }}$ is a 3 -sheeted covering of $\Sigma=\mathbb{P}^{1}$ so $R$ is guaranteed to be even and the objects $\mathcal{O}\left(\lambda \Gamma_{H e t}\right) \equiv \mathcal{L}_{\Gamma}$ and $\mathcal{O}\left(R_{\text {Het }} / 2\right)$ make sense as honest line bundles. This allows us to write

$$
\mathcal{N}_{c, H e t}=\mathcal{L}_{\Gamma} \otimes \mathcal{O}\left(R_{H e t} / 2\right)
$$

As we saw, the connection $A_{\Gamma}$ on $\mathcal{L}_{\Gamma}$ maps directly to $\left.C_{3}\right|_{M 5}$ via the restricted cylinder map (4.34) (4.35). The extra information that we retain about $C_{3}$ that is not found in $G_{4}$ is crucial; we know that $\left.G_{4}\right|_{M 5}=0$ for any M5-instanton that generates a nonzero superpotential coupling without operator insertions. All of the nontrivial bundle behavior depends on the specific configuration of $C_{3}$ on $M 5$. This will be important in section 4.4. As we emphasized at great length in section 2.2, however, the decomposition (4.38) depends on the choice of square root for $\mathcal{O}\left(R_{H e t}\right)$. There are $2^{2 g_{c_{H e t}}}$ such choices in general which are in 1-1 correspondence with the choices of spin structure on $c_{H e t}$. This potentially leads to an ambiguity when we try to define $C_{3}$ in the F-theory dual. Ultimately, the physics should not depend on this ambiguity and indeed we will see that the partition function of the M5 instanton is insensitive to it.

\footnotetext{
${ }^{22}$ The half-integrality of $\lambda \gamma_{H e t}$ matches with the half-integrality of $G_{4}$ that follows from (3.5) and the fact that $c_{2}\left(Y_{4}, \mathbb{Z}\right)$ is typically odd.
} 


\subsection{Heterotic Right-Movers and M5 Fermi Zero Modes}

Before getting to the $C_{3}$-dependence, let us briefly discuss the matching of heterotic right-moving fermi zero modes with the fermi zero modes of the corresponding M5 instanton. Recall that the 'non-universal' right-moving fermi zero modes of the heterotic worldsheet instanton were counted by

$$
2 \times\left[h^{0}\left(\Sigma, N_{\Sigma / B_{2}}\right)+h^{1}\left(\Sigma, N_{\Sigma / B_{2}}\right)+h^{0}(\Sigma, \mathcal{O})+h^{1}(\Sigma, \mathcal{O})\right]
$$

while the corresponding zero modes of an M5 instanton are counted by

$$
2 \times\left[h^{0}\left(D 3, N_{D 3 / B_{3}}\right)+h^{1}\left(D 3, N_{D 3 / B_{3}}\right)+h^{2}\left(D 3, N_{D 3 / B_{3}}\right)+h^{1}(D 3, \mathcal{O})+h^{2}(D 3, \mathcal{O})\right]
$$

To relate these formulae, we first recall that the base $B_{3}$ is a $\mathbb{P}^{1}$-fibration (4.1)

$$
B_{3}=\mathbb{P}(\mathcal{O} \oplus N)
$$

This means that the base $D 3$ of our M5 instanton is a $\mathbb{P}^{1}$ fibration over $\Sigma$ given by

$$
D 3=\mathbb{P}\left(\left.\mathcal{O} \oplus N\right|_{\Sigma}\right) \quad \nu: D 3 \rightarrow \Sigma
$$

and, furthermore, that the normal bundle $N_{D 3 / B_{3}}$ is the pullback of the normal bundle of $\Sigma$ in $B_{2}$

$$
N_{D 3 / B_{3}}=\nu^{*} N_{\Sigma / B_{2}}
$$

We can now use Leray to relate cohomologies on $D 3$ to cohomologies on $\Sigma$

$$
\begin{aligned}
& H^{0}\left(D 3, N_{D 3 / B_{3}}\right)=H^{0}\left(\Sigma, R^{0} \nu_{*} N_{D 3 / B_{3}}\right) \\
& H^{1}\left(D 3, N_{D 3 / B_{3}}\right)=H^{0}\left(\Sigma, R^{1} \nu_{*} N_{D 3 / B_{3}}\right)+H^{1}\left(\Sigma, R^{0} \nu_{*} N_{D 3 / B_{3}}\right) \\
& H^{2}\left(D 3, N_{D 3 / B_{3}}\right)=H^{1}\left(\Sigma, R^{1} \nu_{*} N_{D 3 / B_{3}}\right) .
\end{aligned}
$$

For $N_{D 3 / B_{3}}=\nu^{*} N_{\Sigma / B_{2}}$, the direct image sheaves are particularly simple

$$
R^{0} \nu_{*} N_{D 3 / B_{3}}=N_{\Sigma / B_{2}} \quad R^{1} \nu_{*} N_{D 3 / B_{3}}=0
$$

where $N$ is the bundle from (4.41). This leads to

$$
\begin{aligned}
& H^{0}\left(D 3, N_{D 3 / B_{3}}\right)=H^{0}\left(\Sigma, N_{\Sigma / B_{2}}\right) \\
& H^{1}\left(D 3, N_{D 3 / B_{3}}\right)=H^{1}\left(\Sigma, N_{\Sigma / B_{2}}\right) \\
& H^{2}\left(D 3, N_{D 3 / B_{3}}\right)=0 .
\end{aligned}
$$

Repeating this exercise with $N_{D 3 / B_{3}}$ replaced by $\mathcal{O}$ we have

$$
\begin{aligned}
& H^{1}(D 3, \mathcal{O})=H^{1}(\Sigma, \mathcal{O}) \\
& H^{2}(D 3, \mathcal{O})=H^{2}(\Sigma, \mathcal{O})
\end{aligned}
$$

so that the M5 zero modes (4.40) are counted by

$$
2 \times\left[h^{0}\left(\Sigma, N_{\Sigma / B_{2}}\right)+h^{1}\left(\Sigma, N_{\Sigma / B_{2}}\right)+h^{0}(\Sigma, \mathcal{O})+h^{1}(\Sigma, \mathcal{O})\right]
$$

which is equivalent to (4.39). Concluding, the M5-instanton Fermi zero modes map to right moving fermi zero modes of heterotic worldsheet instantons in the case of duality. 


\subsection{Heterotic Pfaffian and the Chiral 2-form Partition Func- tion}

We finally turn to the contributions from left-movers to our heterotic instanton and the chiral 2-form to the M5. Recall that left-moving fermions contribute $\operatorname{Pfaff}\left(\Sigma, D_{-, V_{\Sigma}}\right)$, the Pfaffian of the Dirac operator on $\Sigma$ coupled to the bundle $V_{\Sigma}=\left.V\right|_{\Sigma}$. Because $V_{\Sigma}$ is the pushforward of a line bundle on the covering curve $c_{H e t}$

$$
V_{\Sigma}=p_{c, \text { Het* }} \mathcal{N}_{c, H e t}
$$

the study of fermions on $\Sigma$ coupled to the non-Abelian bundle $V_{\Sigma}$ can be lifted to the study of fermions on $c_{H e t}$ coupled to an Abelian one. In particular, we saw that the zero modes of $D_{-, V_{\Sigma}}$ on $\Sigma$ lifted to elements of

$$
H^{p}\left(c_{H e t}, \mathcal{N}_{c, H e t} \otimes p_{c, H e t}^{*} K_{\Sigma}^{1 / 2}\right)=H^{p}\left(c_{H e t}, K_{c, H e t}^{1 / 2} \otimes \mathcal{L}_{\Gamma}\right)
$$

on $c_{\text {Het }}$ where we introduce $\mathcal{L}_{\Gamma}$ by taking advantage of the decomposition (4.38)

$$
\mathcal{N}_{c, H e t}=\mathcal{L}_{\Gamma} \otimes \mathcal{O}\left(R_{H e t} / 2\right)
$$

along with the fact that

$$
K_{c, H e t}^{1 / 2}=\mathcal{O}(R / 2) \otimes p_{c, H e t}^{*} K_{\Sigma}^{1 / 2}
$$

is a theta characteristic (i.e. square root of the canonical bundle of) of $c_{H e t}$. This allows us to compute the Pfaffian of interest as

$$
\operatorname{Pfaff}\left(\Sigma, D_{-, V_{\Sigma}}\right) \sim \operatorname{Pfaff}\left(c_{H e t}, D_{-, \mathcal{L}_{\Gamma}}\right) .
$$

We saw in section 2.2 that $\operatorname{Pfaff}\left(c_{H e t}, D_{-, \mathcal{L}_{\Gamma}}\right)=0$ unless $\operatorname{deg}\left(\mathcal{L}_{\Gamma}\right)=0$, in which case it varies with $\mathcal{L}_{\Gamma}$ as a holomorphic section of a line bundle on $\mathcal{J}_{0}\left(c_{\mathrm{Het}}\right)$, the space of degree 0 bundles on $c_{H e t}$. On general grounds, the Pfaffian of free fermions coupled to a flat bundle $\mathcal{L}_{\Gamma}$ on a curve $c_{H e t}$ is proportional to one of the $2^{2 g_{c_{H e t}}} \theta$ functions on $\mathcal{J}_{0}\left(c_{\text {Het }}\right)$; the trick is determining the right one. The ambiguity here is an artificial one that arose from our insistence on splitting $\mathcal{N}_{c, H e t}$ according to (4.51). Looking instead at $H^{p}\left(c_{H e t}, \mathcal{N}_{c, H e t} \otimes p_{c, H e t}^{*} K_{\Sigma}^{1 / 2}\right)$, we were able to conclude that

$$
\operatorname{Pfaff}\left(\Sigma, D_{-, V_{\Sigma}}\right) \sim \theta_{R}\left(\mathcal{N}_{c, H e t} \otimes p_{c, H e t}^{*} K_{\Sigma}^{1 / 2}\right)
$$

where $\theta_{R}$ is the Riemann $\theta$ function, that is the distinguished holomorphic section on $\mathcal{J}_{g-1}\left(c_{H e t}\right)$ whose vanishing locus is precisely the set of degree $g_{c_{H e t}}-1$ bundles that admit holomorphic sections. The split (4.51) simply means that $\operatorname{Pfaff}\left(\Sigma, D_{-, V_{\Sigma}}\right) \sim$ $\operatorname{Pfaff}\left(c_{H e t}, D_{-, \mathcal{L}_{\Gamma}}\right)$ is a translate of the Riemann $\theta$ function by the spin structure $K_{c_{H e t}}^{1 / 2}$

$$
\operatorname{Pfaff}\left(\Sigma, D_{-, V_{\Sigma}}\right) \sim \theta_{R}\left(K_{c, H e t}^{1 / 2} \otimes \mathcal{L}_{\Gamma}\right)=\theta_{-K_{c_{H e t}}^{1 / 2}}\left(\mathcal{L}_{\Gamma}\right) .
$$

The ambiguity in our definitions of $K_{c, H e t}^{1 / 2}$ and $\mathcal{L}_{\Gamma}$ is absent in the first term but present in the second because we use $K_{c_{H e t}}^{1 / 2}$ to translate $\theta_{R}$. 
Given this discussion, one might wonder why $\mathcal{L}_{\Gamma}$ is ever introduced at all. Indeed, it brings the added complication of keeping track of the spin structure and ensuring that any consistent choice we can make does not impact the physics. Of course, we saw in section 4.2 that $\mathcal{L}_{\Gamma}$ is crucially important in the context of heterotic/F-theory duality because it is this quantity that determines the 3 -form $C_{3}$ on the dual M5 instanton. When we construct the map

$$
C_{3}=\iota_{M 5, c y l *} p_{M 5, c y l}^{*} A_{\Gamma}
$$

we are really defining a map from the space of flat bundles on $c_{H e t}$ to the space of 'flat $C_{3}$ 's' on $M 5$. That is, we are defining a map

$$
f_{\text {cyl }}: \mathcal{J}_{0}\left(c_{\text {Het }}\right) \rightarrow \mathcal{J}(M 5)
$$

where we recall that $\mathcal{J}(M 5)=H^{3}(M 5, \mathbb{R}) / H^{3}(M 5, \mathbb{Z})$ is the intermediate Jacobian. This map can be complicated, but $f_{c y l}$ maps $\theta$ divisors to $\theta$ divisors so the $2^{2 g_{c_{H e t}}}$ different $\theta$ functions, $\theta_{-K_{c, H e t}^{1 / 2}}\left(\mathcal{L}_{\Gamma}\right)$ on $\mathcal{J}_{0}\left(c_{H e t}\right)$ are identified with the $2^{2 g_{c_{H e t}}}$ different $\theta$ functions $\theta_{a}\left(C_{3}\right)$ on $\mathcal{J}(M 5)$. That the heterotic Pfaffian maps to a $\theta$ function on $\mathcal{J}(M 5)$ is exactly what we need. The heterotic left-movers map to the chiral 2 -form under heterotic/F-theory duality so we have

$$
\operatorname{Pfaff}\left(\Sigma, D_{-, V_{\Sigma}}\right) \leftrightarrow \mathcal{Z}_{\phi}
$$

The 2-form partition function $\mathcal{Z}_{\phi}$ must be proportional to a $\theta$ function on $\mathcal{J}(M 5)$ in order to ensure that the full M5 partition function (3.28) is gauge invariant. Though the 'right' $\theta$ function can be determined from the spin structures of $M 5$ and $Y_{4}^{\prime}$ via Witten's procedure [66], we see that the task is much easier in models with a heterotic dual.

Heterotic duality actually teaches something that goes beyond a practical tool for picking the 'right' $\theta$ function. We see clearly in this setting that the choice of $\theta$ function is intricately connected to the specification of $C_{3}$ itself. This is a level of detail about the 3 -form that is easy to miss if we only specify a $G$-flux by giving the homology class of a holomorphic surface. We get a handle on the heterotic Pfaffian, or equivalently the chiral 2-form partition function, when we are able to talk about the line bundle $\mathcal{N}_{c, \text { Het }}$ or, equivalently, its pullback to $c_{c y l}, \mathcal{N}_{M 5, \text { cyl }}$. This line bundle is the object that unequivocally controls the vanishing of $\operatorname{Pfaff}\left(\Sigma, D_{-, V_{\Sigma}}\right) \sim \mathcal{Z}_{\phi}$ and the spin structure ambiguity arises because a choice of $K_{c, \text { Het }}^{1 / 2}$ is needed to construct $C_{3}$ from $\mathcal{N}_{M 5, c y l}$. In a sense, then, $\mathcal{N}_{M 5, c y l}$ is the object on which the physics most directly depends. We see something similar in the $E_{6}$ gauge theory where the spectrum is determined by cohomologies of $\mathcal{N}_{l o c}$ without any ambiguity from spin structures. It is only when we try to work in terms of quantities derived from $\gamma$ that depend on the separation (2.10) that we run into trouble. Of course any trouble can be fixed by taking care to keep track of the requisite spin structures in the problem. It seems far simpler to work directly with the bundle $\mathcal{N}_{c y l}$, though, as it is the object that directly affects the physics. Any spin structure dependence can be absorbed into a twist of the map from the connection $A_{c y l}$ of $\mathcal{N}_{c y l}$ to $C_{3}$. 


\section{Instanton Prefactors in F-theory}

We have discussed at length how one might think of instanton prefactors in F-theory from an M-theory and a heterotic point of view, and also the relationship between the two. At a fundamental level, the instanton prefactor in F-theory is determined by configurations of $C_{3}$ in the defining $d=3$ M-theory compactification, which are elements of the intermediate Jacobian $\mathcal{J}(M 5)$. If the M-theory flux $G_{4}$ is cohomologically trivial on $M 5$, the prefactor is the partition function $\mathcal{Z}_{\phi}$ and is given by a theta divisor on the intermediate Jacobian. In the case of an M5-instanton which is dual to a heterotic worldsheet instanton, the prefactor is dependent via duality on the fermionic left-movers which couple to the gauge bundle $V$ that breaks $E_{8}$ to the GUT group. Recalling that

$\mathcal{L}_{A} \equiv K_{c_{H e t}}^{1 / 2} \otimes \mathcal{L}_{\Gamma}$, these modes are counted by the Hodge numbers $h^{i}\left(c_{H e t}, \mathcal{L}_{A}\right)$ where $c_{H e t}$ is a spectral cover of the instanton curve $\Sigma$. From sections 2.2 and 3.2 .2 , in fact, we know how to determine the partition function of these modes and, correspondingly, the partition function of the M5 chiral 2-form, both of which correspond to $\theta$ functions. While these issues of $\theta$ functions are important, they are typically not important for understanding how the prefactor varies as a function of the available moduli. The reason for this is that the full domain of the $\theta$ function, either $\mathcal{J}_{g-1}\left(c_{H e t}\right)$ for our heterotic instantons or $\mathcal{J}(M 5)$ for our $M 5$ instantons, is typically not accessible, since the connection $A_{\Gamma}$ and three-form $C_{3}$ are restricted from global objects defined outside of the instanton geometry. Moreover, we would like to express the prefactor in terms of extrinsic moduli which explicitly determine the seven-brane structure of the compactification. This depends upon the embedding of $c_{H e t}$ (or $c_{l o c}$ in what follows) into the geometry, as opposed to the theta functions that are intrinsic to $c_{H e t}$. The relationship between the 'extrinsic' and 'intrinsic' viewpoints is discussed in 63] for heterotic worldsheets.

Study of this line bundle cohomology allows one to determine how the M5-instanton prefactor depends on the moduli of $V$ via the algebro-geometric techniques of [59], and is equivalent to the study of the vector bundle cohomology $h^{i}\left(\Sigma,\left.V\right|_{\Sigma} \otimes \mathcal{O}_{\Sigma}(-1)\right)$. In this picture, the moduli in the prefactor appear in the defining equation of $c_{H e t}$ and also explicitly in the defining equation of $Y_{4}$; thus, it is a simple matter to see the sevenbrane dependence of the prefactor once it is computed. However, the appearance of seven-brane physics is rather unintuitive when computed via the study of line bundle cohomology on $c_{H e t}$, since this curve exists away from the GUT stack and only in the case of a heterotic dual. In particular the type IIb intuition of zero modes localized near branes is lacking, a fact which we would like to rectify.

In this section we note that in the heterotic case, the dependence of the prefactor on the bundle $V$ can be described via the study of line bundle cohomology on another, isomorphic curve $c_{l o c}$. This has an analogue in F-theory that naturally appears in the GUT geometry. We propose this as the right way to compute the moduli dependence of the F-theoretic prefactor. The curve $c_{l o c}$ is a multi-sheeted cover of the curve $\Sigma$ in $B_{3}$ where the GUT stack intersects the instanton, and thus the associated zero modes admit a natural IIb interpretation in terms of 3-7 strings. Moreover, the relevant line bundle cohomology on $c_{l o c}$ is isomorphic to the discussed line bundle cohomology on $c_{H e t}$ when a dual exists, and thus the prefactors are equivalent. However, the curve $c_{l o c}$ has the advantage that it exists even in the absence of a heterotic dual. We will make a precise conjecture for how line bundle cohomology on $c_{l o c}$ is related to the worldvolume 
theory of the M5 brane and theta divisors on the intermediate Jacobian $\mathcal{J}(M 5)$. We will also discuss the concrete steps necessary to set up the calculation of the prefactor, which will be utilized in section 8 .

We will first discuss how to compute the prefactor and why it makes sense from type IIb intuition and also heterotic duality. We will then make a mathematically precise statement regarding the relationship to the M5 brane worldvolume theory. We will finish with a concrete discussion of how to set up the computation for a given F-theory base, $B_{3}$.

\subsection{Intuition and a Proposal: What to Compute}

Recall that when a heterotic dual exists the dependence of the M5-instanton prefactor on the moduli of $V$ can be determined by the study of $\operatorname{Pfaff}\left(\Sigma, D_{-, V_{\Sigma}}\right)$. In principle, one could study $\operatorname{Pfaff}\left(\Sigma, D_{-, V_{\Sigma}}\right) \sim \theta_{R}\left(\mathcal{N}_{c, \text { Het }} \otimes p_{c, \text { Het }}^{*} K_{\Sigma}\right)$ for any choice of line bundle $\mathcal{N}_{c, \text { Het }}$ of the right degree, but the line bundle $\mathcal{N}_{c, \text { Het }}$ we study must arise from the restriction of a line bundle $\mathcal{N}_{\text {Het }}$ on $\mathcal{C}_{\text {Het }}$. That is,

$$
\mathcal{N}_{c, H e t}=\left.\mathcal{N}_{H e t}\right|_{c_{H e t}} .
$$

Similarly, the 3-form $C_{3}$ on $M 5$ must arise as the restriction of a well-defined configuration for $C_{3}$ on $Y_{4}^{\prime}$. We are interested in the vanishing locus and the behavior of $\operatorname{Pfaff}\left(\Sigma, D_{-, V_{\Sigma}}\right) \sim \mathcal{Z}_{\phi}$ as one varies the complex structure moduli of $\mathcal{C}_{H e t}$ and $Y_{4}^{\prime}$. We reviewed the procedure of [59,68, 69] for computing this dependence, at least on the heterotic side, in section 2.3. This approach is based on transforming the non-Abelian problem to an Abelian one via

$$
\operatorname{Pfaff}\left(\Sigma, D_{-, V_{\Sigma}}\right) \sim \operatorname{Pfaff}\left(c_{H e t}, D_{-, \mathcal{L}_{\Gamma}}\right)
$$

and computing Pfaff $\left(c_{H e t}, D_{-, \mathcal{L}_{\Gamma}}\right)$ by standard techniques. The end result is that the heterotic Pfaffian is determined by the partition function of free fermions on $c_{H e t}$ coupled to a degree zero line bundle $\mathcal{L}_{\Gamma}$. The techniques of [59] computes this via study of the bundle $\mathcal{L}_{A} \equiv \mathcal{L}_{\Gamma} \otimes K_{c_{H e t}}^{1 / 2}$, and when this bundle has a section the prefactor has a zero. By duality, this Pfaffian should capture the behavior of the chiral 2-form partition function as well.

This leads to a natural question: why should the partition function of an M5 instanton have anything to do with fermions on some auxiliary curve $c_{H e t}$ that emerges in the stable degeneration limit? One encounters a similar question when studying the spectrum of the $E_{6}$ gauge theory in the context of heterotic/F-theory duality. There, the heterotic computations are cohomologies on a curve in $Z_{H e t}$, which sits at $W=0$. This occurs away from the $E_{6}$ singular locus at $Z=0$, where the $E_{6}$ charged degrees of freedom are localized. The resolution is that the proper computation on the F-theory side involves cohomologies on curves in $\mathcal{C}_{l o c}$, which sits inside the $E_{6}$ singular fibration over $Z=0$. When a heterotic dual exists, it happens that the spectral divisor $\mathcal{C}_{F}$ becomes a $\mathbb{P}^{1}$ fibration, $\mathcal{C}_{c y l}$, and this provides a second copy of $\mathcal{C}_{\text {loc }}=\mathcal{C}_{\text {Het }}$ at $W=0$ as the second section. The F-theory computations can be translated from $Z=0$ to $W=0$ using this $\mathbb{P}^{1}$ fibration and, in fact, the copy of $\mathcal{C}_{\text {loc }}=\mathcal{C}_{\text {Het }}$ at $W=0$ is easier to work with because we don't have to pass to the resolution of $Y_{4}$ to see it. For emphasis, 
the conclusion is that the charged degrees of freedom are localized on the GUT stack, but when a heterotic dual exists one could instead study an isomorphic curve and line bundle away from the GUT stack inside the heterotic threefold.

In a similar way, a copy of the curve $c_{H e t}$ also sits above the $E_{6}$ singular locus at $Z=0$. It is just the curve $c_{l o c}=p_{l o c}^{*} \Sigma$, inside the Higgs bundle spectral cover that emerges from the restriction $\mathcal{C}_{l o c}=\left.\mathcal{C}_{c y l}\right|_{Z=0}$. As a reminder, $\mathcal{C}_{l o c}$ is a multi-sheeted cover of $B_{2}$ and $c_{l o c}$ is the multi-sheeted cover of $\Sigma \subset B_{2}$ derived from $\mathcal{C}_{l o c}$ by restriction. While fermions on some curve $c_{H e t}$ at $W=0$ do not have an obvious physical interpretation, fermions on $c_{l o c}$, which sits above the curve $\Sigma$ where $M 5$ meets the $E_{6}$ branes, do: they should correspond to an analog of 3-7 strings! This suggestion is not unique to us; the curve $c_{H e t}$ has even been referred to as $\Sigma_{37}$ in [49] and related cohomologies were also discussed in [48]. Our precise description of the compact relation of $\mathcal{C}_{\text {loc }}=\left.\mathcal{C}_{c y l}\right|_{\pi^{*} B_{2}}$, however, allows us to identify the compact curve $c_{l o c}$ for generic $E_{6}$ (or $S O(10)$ or $S U(5))$ models in a way that reproduces $c_{l o c}=c_{H e t}$ when a heterotic dual exists. Further, if we identify $p_{l o c, *} \mathcal{N}_{l o c}$ as the gauge bundle $V$ associated to the local model on $B_{2}$, the cohomologies on $c_{l o c}$ are simply counting fermions localized on the intersection $B_{2} \cap D 3=\Sigma$ that couple to $V_{\Sigma}=\left.V\right|_{\Sigma}$

$$
H^{p}\left(c_{l o c}, \mathcal{L}_{A}\right) \equiv H^{p}\left(c_{l o c}, p_{c, l o c}^{*} K_{\Sigma}^{1 / 2} \otimes \mathcal{N}_{c, l o c}\right)=H^{p}\left(\Sigma, K_{\Sigma}^{1 / 2} \otimes V_{\Sigma}\right) .
$$

This is exactly how we would expect 3-7 modes to appear from the perspective of the worldvolume theory on $B_{2}$.

With our proper, compact description of the curve $c_{l o c}$ in hand, we propose that the cohomologies (5.3) control the vanishing of the M5 partition function for any M5 instanton in the $E_{6}$ models we consider. More generally, if we have multiple non-Abelian gauge groups that can be described in the local framework of $S U(n)$ Higgs bundles, we expect the vanishing to be controlled by cohomologies on all of the relevant $23 c_{l o c}$ 's provided the M5 avoids any loci where non-Abelian singularities intersect. Specifically, the proposed relationship between modes on $c_{l o c}$ and the prefactor $A$ is

$$
h^{i}\left(c_{l o c}, \mathcal{L}_{A}\right) \neq 0 \quad \Rightarrow \quad A=0
$$

which is similar to the statement regarding modes on $c_{H e t}$ and the Pfaffian prefactor. The motivation for this proposal is clear. If the vanishing of the M5 instanton partition function is controlled by cohomologies on $c_{l o c}$, it suggests that the physics responsible for this vanishing is captured by the local geometry near $B_{2}$ and, correspondingly, should not depend on whether $B_{2}$ is sitting inside a $\mathbb{P}^{1}$ fibration or not. Thus, we have a prescription for computing the dependence of the instanton prefactor on seven-brane moduli, even in the absence of a heterotic dual. This gives a very powerful tool, computationally equivalent to the computations in [59], that could be applied in a number of phenomenological models. After all, everything we say in this paper about $E_{6}$ can be trivially extended to the phenomenologically interesting cases of $S O(10)$ and $S U(5)$.

We emphasize that while this prescription is physically sensible from the type IIb point of view and isomorphic to the prescription for heterotic worldsheet instantons in the case of duality, the relationship to the M5 brane worldvolume theory could be put on more solid footing, and hence the result is still conjectural. We will now address some of this issue.

\footnotetext{
${ }^{23}$ Including those corresponding to the associated bundles $\wedge^{k} V$.
} 


\subsubsection{Mathematical Relationship to the M5-brane Theory}

Let us state in a mathematically precise way what this conjecture would mean from the point of view of the M5-brane worldvolume theory, at least for the $E_{6}$ models discussed in this paper. The M5 worldvolume is an elliptic fibration with section over a surface $D 3$ in $B_{3}$ that exhibits an $E_{6}$ singularity along the curve $\Sigma$ where $D 3$ meets $B_{2}$. We can describe $M 5$ by an equation of the form (3.2)

$$
y^{2}=x^{3}+f x z^{4} v^{4}+g z^{6} v^{6}+z^{2} v^{3}\left[\tilde{b}_{0} z^{3} v^{3}+\tilde{b}_{2} z v x+\tilde{b}_{3} y\right]
$$

where we have restricted the sections $b_{q}$ on $B_{3}$ should be restricted to $D 3 \subset B_{3}$, giving the sections $\tilde{b}_{q}$. Inside $M 5$, we then consider the restriction of the spectral divisor $\mathcal{C}_{F}$ (3.4)

$$
c_{F}=\left.\mathcal{C}_{F}\right|_{M 5}
$$

Recall that $\mathcal{C}_{F}$ is a distinguished 3 -sheeted cover of $B_{3}$ inside $Y_{4}$ that is given in terms of the data in (5.5) by

$$
c_{F}: \quad \tilde{b}_{0} z^{3} v^{3}+\tilde{b}_{2} z v x+\tilde{b}_{3} y=0
$$

less the components that coincide with the section. Correspondingly, the cover $c_{F}$ of $D 3$ inside $M 5$ is also distinguished and its restriction to $\pi^{*} \Sigma$ gives us the curve $c_{l o c}$

$$
c_{l o c}=\left.c_{F}\right|_{\pi^{* \Sigma}}
$$

which is a 3 -sheeted cover of $\Sigma$. This curve is isomorphic to $c_{H e t}$ when a heterotic dual exists. The role of $c_{F}$ is the usual one: line bundles on $c_{F}$ simultaneously determine the 3 -form $C_{3}$ on $M 5$ and bundle data $\mathcal{N}_{c, l o c}$ in the local model.

Our conjecture about vanishing loci is a statement about $\theta$ divisors in $\mathcal{J}_{g-1}\left(c_{l o c}\right)$ and $\mathcal{J}(M 5)$. More specifically, let $\mathcal{A}$ denote the subset of $\operatorname{Pic}\left(c_{F}\right)$ given by

$$
\mathcal{A}=\left\{\mathcal{N}_{c, F} \in \operatorname{Pic}\left(c_{F}\right)\left|\mathcal{N}_{c, F}\right|_{c_{l o c}} \otimes p_{c, l o c}^{*} K_{\Sigma}^{1 / 2} \in \mathcal{J}_{g-1}\left(c_{l o c}\right)\right\}
$$

We have the obvious map

$$
h_{l o c}: \mathcal{A} \rightarrow \mathcal{J}_{g-1}\left(c_{l o c}\right) \quad h_{l o c}:\left.\mathcal{N}_{c, F} \mapsto \mathcal{N}_{c, F}\right|_{c_{l o c}} \otimes p_{c, l o c}^{*} K_{\Sigma}^{1 / 2}
$$

which determines the local data corresponding to a bundle $\mathcal{N}_{c, F}$. We also have the analog of the map (4.33) for determining $C_{3}$

$$
h_{M 5}: \mathcal{A} \rightarrow \mathcal{J}(M 5) \quad h_{M 5}: \mathcal{N}_{c, F} \mapsto \iota_{M 5, c *}\left(A_{M 5, c}-\frac{1}{2} A_{\hat{R}}\right)
$$

Here $\iota_{M 5, c}: c_{F} \rightarrow M 5$ is the embedding map, $A_{M 5, c}$ is a holomorphic connection on $\mathcal{N}_{c, F}$, and $A_{\hat{R}}$ is a holomorphic connection on the bundle $\mathcal{L}_{\hat{R}}=\left.\mathcal{L}_{\hat{r}}\right|_{M 5}$. Our conjecture

\footnotetext{
${ }^{24}$ We can define $\mathcal{L}_{\hat{R}}$ intrinsically on $M 5$ as follows. Start with the bundle $\mathcal{O}_{c_{F}}\left(R_{F}\right)$ where $R_{F}$ is the ramification divisor of the covering $c_{F} \rightarrow D 3$. Now, consider the normal bundle $N_{c_{l o c} / c_{F}}$ and let $L^{\prime}$ denote the bundle obtained by removing the part of $N_{c_{l o c} / c_{F}}$ that is pulled back from $D 3$. We take $\mathcal{L}_{\hat{R}}=\mathcal{O}_{c_{F}}\left(R_{F}\right) \otimes L^{\prime},-1$ and it has the property that $\left.\mathcal{L}_{\hat{R}}\right|_{c_{l o c}}=\mathcal{O}_{c_{l o c}}\left(r_{l o c}\right)$ with $r_{l o c}$ the ramification divisor of the covering $c_{l o c} \rightarrow \Sigma$.
} 
is that the M5 instanton partition function vanishes for any choice of $\mathcal{N}_{c, F}$ that maps to $\Theta_{R} \subset \mathcal{J}_{g-1}\left(c_{l o c}\right)$ under $h_{l o c}$. If we let $\mathcal{A}_{\text {vanish }}$ denote the conjectured vanishing locus

$$
\mathcal{A}_{\text {vanish }}=\left\{\mathcal{N}_{c, F} \in \mathcal{A} \mid h_{\text {loc }}\left(\mathcal{N}_{c, F}\right) \in \Theta_{R} \subset \mathcal{J}_{g-1}\left(c_{l o c}\right)\right\}
$$

then our claim is that there exists a theta divisor $\Theta_{M 5 \text {,vanish }} \subset \mathcal{J}(M 5)$ such that

$$
h_{M 5}\left(\mathcal{A}_{\text {vanish }}\right) \subset \Theta_{M 5, \text { vanish }}
$$

The $\theta$ function corresponding to $\Theta_{M 5, v a n i s h}$ should be the 'right' one that controls the partition function of the chiral 2-form on $M 5$.

What we describe here is a 'strong form' of the conjecture. It should be noted that physics only requires a weaker version in which we specify $\mathcal{C}_{F}$ as well as its restriction $c_{F}$ and consider only the subsets of $\mathcal{A}$ and $\mathcal{A}_{\text {vanish }}$ that correspond to bundles on $c_{F}$ that descend from bundles on $\mathcal{C}_{F}$.

\subsection{Setting Up the Computation}

In the end, to compute the instanton prefactor we compute moduli-dependent line bundle cohomology on a spectral curve $c_{l o c}$, which is the restriction of the local model spectral cover $\mathcal{C}_{l o c}$ to the instanton worldvolume. We will utilize the fact that $\mathcal{C}_{l o c}$ is a compact spectral cover inside an auxiliary elliptic Calabi-Yau threefold $\pi_{3, F}: Z_{3, F} \rightarrow B_{2}$, as discussed in section 3.1.1, which in turn gives $c_{l o c}$ as a curve inside the elliptic surface $\mathcal{E} \equiv \pi_{3, F}^{*} \Sigma$. The advantage of this approach is that it simplifies computations. We will see that one ends up computing the cohomology a line bundle on $c_{l o c}$ via a Koszul sequence from a line bundle on $\mathcal{E}$. Though this computation is in an F-theory compactification which may or may not have a heterotic dual, the mathematical computation is identical to those performed in [69], to which we refer the reader for detailed examples. Instead, here we will describe how to obtain the topological data necessary to set up a computation along the lines of [69]. In particular, one must know that structure of the elliptic surface $\mathcal{E}$, the class of $c_{l o c}$ inside $\mathcal{E}$, and how the bundle $\mathcal{L}_{A}$ is obtained via a restriction from a line bundle on $\mathcal{E}$. The computation of cohomology on $c_{l o c}$ as a divisor in $\mathcal{E}$ is a choice, albeit a convenient one, and we emphasize that it is the cohomology on $c_{l o c}$ which governs the physics, regardless of ambient space.

Recall from 3.1 .1 that $\pi_{3, F}: Z_{3, F} \rightarrow B_{2}$ is the ambient elliptic Calabi-Yau threefold in which $\mathcal{C}_{\text {loc }}$ is a divisor. The class of $\mathcal{C}_{\text {loc }}$ is

$$
\left[\mathcal{C}_{\text {loc }}\right]=n \sigma_{3, F}+\pi_{3, F}^{-1} \eta
$$

where $\eta$ is a curve in $B_{2}$ of class $6 c_{1, B_{2}}+N_{B_{2} \mid B_{3}}$ and $\sigma_{3, F}$ is the section of $Z_{3, F}$. $\Sigma$ is a $\mathbb{P}^{1}$ in $B_{2}$, and is the base of an elliptic surface $\mathcal{E} \equiv \pi_{3, F}^{-1} \Sigma$. For instanton physics, we are interested in the curve $c_{l o c}$. Recalling that $c_{l o c}=\mathcal{C}_{l o c} \cdot \mathcal{E}$ inside $Z_{3, F}$, the class of $c_{l o c}$ inside $\mathcal{E}$ is then

$$
\left[c_{l o c}\right]=\left[n \sigma_{3, F}+\pi_{3, F}^{-1}\right] \cdot \Sigma=n s+r F
$$

where $\left.s \equiv \sigma_{3, F}\right|_{\pi_{3, F}^{-1} \Sigma}$ is the section of the elliptic surface $\mathcal{E}, F$ is the fiber class of $\mathcal{E}$ and $r \equiv \eta \cdot B_{2} \Sigma \in \mathbb{Z}$. From (3.19) we write the defining equation of $c_{l o c}$

$$
f_{c_{l o c}}=\sum_{q=1}^{n} \tilde{b}_{q} v^{n-q} x^{n_{x}} y^{n_{y}}
$$


which just reduces to

$$
f_{c_{l o c}}=\tilde{b}_{0} v^{3}+\tilde{b}_{2} v x+\tilde{b}_{3} y
$$

in the $E_{6}$ case $(n=3)$ that we consider in examples. The sections $\tilde{b}_{q}$ are obtained via restriction of $b_{q}$ to $\mathcal{E}$ and are sections of $\mathcal{O}_{\Sigma}(r-q \chi)$.

Let us now discuss the relevant line bundles on $\mathcal{C}_{l o c}$ and $c_{l o c}$. In both cases, these bundles will be obtained via restriction from bundles on an ambient space of the same name, abusing notation. The line bundle $\mathcal{N}_{l o c}$ on $\mathcal{C}_{l o c}$ can be obtained via restriction from a line bundle on $Z_{3, F}$ of first Chern class

$$
c_{1}\left(\mathcal{N}_{\text {loc }}\right)=\frac{1}{2}\left(n \sigma_{3, F}+\pi_{3, F}^{-1} \eta+\pi_{3, F}^{-1} c_{1, B_{2}}\right)+\lambda\left(n \sigma_{3, F}-\left(\pi_{3, F}^{-1} \eta-n \pi_{3, F}^{-1} c_{1, B_{2}}\right)\right) .
$$

Restricting to $c_{l o c}$ gives a bundle $\left.\mathcal{N}_{c, l o c} \equiv \mathcal{N}_{l o c}\right|_{c_{l o c}}$ which can be obtained via restriction from a line bundle on $\mathcal{E}$ of first Chern class

$$
c_{1}\left(\mathcal{N}_{c, l o c}\right)=\frac{1}{2}(n s+(r+\chi) F)+\lambda(n s-(r-n \chi) F) .
$$

where $\chi \equiv c_{1, B_{2}} \cdot B_{2} \Sigma$. In terms of data on $\mathcal{E}$, the bundle $\mathcal{L}_{A}$ in (5.3) relevant for the study of the prefactor can be written $\left.\mathcal{L}_{A} \equiv \mathcal{N}_{c, l o c} \otimes \mathcal{O}_{\mathcal{E}}(-F)\right|_{c_{l o c}}$, so we have

$$
\left.c_{1}\left(\mathcal{L}_{A}\right)=\left(\lambda+\frac{1}{2}\right) n s+\left[r\left(\frac{1}{2}-\lambda\right)+\chi\left(\frac{1}{2}+n \lambda\right)-1\right)\right] F
$$

and one must compute $h^{i}\left(c_{l o c},\left.\mathcal{L}_{A}\right|_{c_{l o c}}\right)$ in order to determine the prefactor. This is done efficiently via the long exact sequence in cohomology associated to the Koszul sequence

$$
\left.0 \rightarrow \mathcal{L}_{A} \otimes \mathcal{O}_{\mathcal{E}}\left(-c_{l o c}\right) \stackrel{f_{c_{l o c}}}{\longrightarrow} \mathcal{L}_{A} \rightarrow \mathcal{L}_{A}\right|_{c_{l o c}} \rightarrow 0
$$

as in 69. Though the restriction is explicit, remember that we abuse notation and use the name $\mathcal{L}_{A}$ for both the bundle on $\mathcal{E}$ and its restriction to $c_{\text {loc }}$.

Armed with these definitions and an F-theory compactification on a particular base $B_{3}$ and an $E_{6}$ GUT along $B_{2}$, one can scan for divisors in $B_{3}$ or $Y_{4}$ which satisfy the constraints (3.26) and (3.27) sufficient for an instanton on that divisor to correct the superpotential. Knowing the instanton divisor, the numbers $r$ and $\chi$ can be computed directly and together with an appropriately quantized $\lambda$ one has all of the information necessary to compute the dependence of the prefactor on the moduli of $c_{l o c}$. We will make this explicit in the examples of section 8 . One nice feature of this computation is that all of the requisite information can be determined directly from $B_{3}$, allowing for a simple scan over F-theory bases.

As emphasized, the computations we study are in the $E_{6}$ case where $n=3$, but this formalism can also be applied to compute the components of the instanton prefactor in $S U(5)$ GUTs with $n=5$. For cases $n>3$, however, one should in principle also study the zero modes of the "associated bundles". By this we mean that the $\mathcal{L}_{A}$ cohomology on $c_{l o c}$ here is equivalent to the bundle cohomology $V$ on $\Sigma$, but for higher rank bundles one should also study $\wedge^{k} V$ cohomology on $\Sigma$. 


\section{A Prefactor Scan in the Kreuzer-Skarke Database}

In a series of seminal works Kreuzer and Skarke classified all three-dimensional [65] and four-dimensional [76] reflexive polytopes. Associated to every $d$-dimensional reflexive polytope is a $d$-dimensional toric variety which admits a $(d-1)$-dimensional Calabi-Yau hypersurface. To do so, the anticanonical bundle of the toric variety must have a section. While we will not be using this method to construct Calabi-Yau hypersurfaces, the fact that the anticanonical bundle admits a section will be useful for constructing F-theory compactifications.

In this brief section we will perform a scan of a large class of threefold bases studying the statistics of possible instanton corrections to the superpotential. We will do so by systematically collecting the topological data necessary to set up the computation of the instanton prefactor, as outlined in section 5.2. Rather than taking the CalabiYau hypersurface in the $d$-dimensional toric variety $B_{d}$ associated to a $d$-dimensional polytope, we will use the toric varieties $B_{3}$ in the Kreuzer-Skarke list [65] to be threefold bases of F-theory compactifications. That the anticanonical bundle $K_{B_{3}}^{-1}$ admits a section ensures that $f$ and $g$ in the Weierstrass equation (or alternatively the sections in the Tate form) have sections and therefore the F-theory compactification exists.

We perform a simple scan of the Kreuzer-Skarke database as follows:

- Find all fine triangulations of the 4319 reflexive polytopes in [65].

- Study the toric variety associated to each fine triangulation and keep each smooth toric variety as a candidate $B_{3}$.

- For each smooth $B_{3}$, scan the toric divisors for divisors $D 3$ with $\chi\left(D 3, N_{D 3 \mid B_{3}}\right)=0$. These are the candidate instanton divisors in $B_{3}$.

- For each candidate instanton divisor $D 3$, scan through the toric divisors for candidate GUT divisors $B_{2}$ and keep those $B_{2}$ which intersect $D 3$ at a $\mathbb{P}^{1}$ in $B_{3}$. This is $\Sigma$.

- For each pair $\left(D 3, B_{2}\right)$ compute $r$ and $\chi$ as defined in section 5.2. Keep only those with $r>0$ since otherwise $c_{l o c}$ is not defined. Use $r$ and $\chi$ to determine $\mathcal{L}_{A}$ for $n=3$ and $\lambda=\frac{3}{2}$. Together, this data gives the class $c_{l o c}$ and the line bundle $\mathcal{L}_{A}$ and therefore one could compute the prefactor at this point. Write $\left.\mathcal{L}_{A} \equiv \mathcal{O}_{\mathcal{E}}(M s+N F)\right|_{c_{l o c}}$.

- Add $B_{3}$ to the list of toric varieties which realize an instanton with prefactor determined by these values of $r$ and $\chi$.

There are a number of ways that one might generalize or strengthen this scan. One is to consider instanton or GUT divisors which are not toric divisors, defined by the vanishing of a single homogeneous coordinate. Another is to consider different values for $n$ or $\lambda$. For $n=3$ and $\lambda=\frac{1}{2}$, it is easy to show that $\left.\mathcal{L}_{A}\right|_{c_{l o c}}$ almost always has a section and therefore the prefactor vanishes identically. For $n=3$ and $\lambda>\frac{3}{2}$ it is much more likely that one has to add $\overline{D 3}$-branes for the sake of tadpole cancellation.

Having discussed possible generalizations and caveats, let us turn to a discussion of the results of this scan presented in Table1. This table contains the results for KreuzerSkarke database except for polytope 4309, which we discuss on its own. Each row has a unique set of integers $r, \chi, M, N$ which determines the class of $c_{l o c}$ and the line 


\begin{tabular}{cccc|c|c}
$r$ & $\chi$ & $M$ & $N$ & Multiplicity & Comments \\
\hline 1 & 0 & 6 & -2 & 5078 & $A=0$, Ex. 3 of [69] \\
5 & 1 & 6 & -1 & 28267 & pts of E8, Ex. 2 of [69] \\
6 & 1 & 6 & -2 & 32634 & $A=0$, Ex. 3 of section [8. \\
7 & 1 & 6 & -3 & 3217 & not computed \\
8 & 1 & 6 & -4 & 55 & not computed \\
11 & 2 & 6 & -2 & 30670 & not computed \\
12 & 2 & 6 & -3 & 5996 & not computed \\
13 & 2 & 6 & -4 & 168 & not computed \\
16 & 3 & 6 & -2 & 4284 & not computed \\
17 & 3 & 6 & -3 & 2827 & not computed \\
18 & 3 & 6 & -4 & 155 & not computed \\
19 & 3 & 6 & -5 & 7 & not computed \\
23 & 4 & 6 & -4 & 33 & not computed
\end{tabular}

Table 1: Results of a prefactor scan in the Kreuzer-Skarke list of toric threefolds, not including threefolds derived from polytope 4309. The prefactors associated to the first two rows of data are computed elsewhere in the literature. This scan is for $E_{6} \operatorname{GUTs}(n=3)$ and $\lambda=\frac{3}{2}$.

\begin{tabular}{cccc|c|c}
$r$ & $\chi$ & $M$ & $N$ & Multiplicity & Comments \\
\hline 1 & 0 & 6 & -2 & 24576 & $A=0$, Ex. 3 of $[69]$ \\
5 & 1 & 6 & -1 & 98304 & pts of $E_{8}$, Ex. 2 of $[69]$ \\
6 & 1 & 6 & -2 & 73728 & $A=0$, Ex. 3 of section $[8$. \\
11 & 2 & 6 & -2 & 73728 & not computed \\
16 & 3 & 6 & -2 & 24576 & not computed
\end{tabular}

Table 2: Results of a prefactor scan which utilizing the toric varieties of all fine triangulations of Kreuzer-Skarke polytope 4309 as F-theory base manifolds $B_{3}$. The prefactors associated to the first two rows of data are computed elsewhere in the literature. This scan is for $E_{6}$ GUTs $(n=3)$ and $\lambda=\frac{3}{2}$.

bundle $\mathcal{L}_{A}$ on it. That is, $r, \chi, M$, and $N$ are the topological quantities which are the inputs necessary to the compute the prefactor. The first row corresponds to a prefactor which has already been computed in the heterotic literature and it is identically zero. The second is the prefactor of the F-theory GUT in section 8.2 and was also previously computed in the heterotic literature. This prefactor vanishes if and only if there exists a point of $E_{8}$ enhancement in the instanton worldvolume. The third example is a new prefactor, computed in section 8.3 of this paper. It is identically zero. To the authors' knowledge, none of the other examples have been computed in the literature. It is worth noting that the computational complexity of each example goes up as one progresses down the rows. This can roughly be seen from the fact that $r$ is increasing, which means that there are more moduli appearing in $f_{c_{l o c}}$, and the fact that $\chi$ is increasing, which roughly means that the size of a moduli-dependent matrix is increasing. The determinant of this matrix is the prefactor.

The primary reason for presenting these results, though, is that there are over 
100, 000 prefactors, and only thirteen of them are unique as polynomial functions. To be more specific, every row corresponds to the computation of a matrix determinant and yields a moduli-dependent polynomial function $f(\phi)$ which is the instanton prefactor by holomorphy. There are only thirteen explicit computations to perform, and given those polynomials one could substitute in the appropriate moduli in the over 100,000 examples. We find this degeneracy interesting.

This has clear implications for moduli stabilization. First, the prefactors are highly structured and there are not very many unique functions. Second, many of them are identically zero which would greatly affect Kähler moduli stabilization. Such a prefactor can be the determinant of a highly non-trivial matrix in moduli, as exemplified in section 8.3, and determining that there is a zero mode and therefore an identically zero prefactor requires an explicit construction of the moduli map. Though this occurred while studying a seven-brane dependent instanton prefactor in F-theory, this is a more generic mathematical phenomenon and should occur in other compactifications as well. Therefore, one should not simply assume an $\mathcal{O}(1)$ prefactor, particularly given the fact that identically zero prefactors were very common in our scan. Third, there are 28,267 examples where the prefactor vanishes if and only if there exists a point of $E_{8}$ in the instanton worldvolume. It would be interesting to compute the remaining prefactors in the list and to study these ideas further.

Finally, the analogous results for polytope 4309 are given in table 2. We see that compactifications utilizing the toric variety associated to a fine triangulation of this polytope as F-theory bases $B_{3}$ give a very high multiplicity of instanton prefactors. These results have been excluded from table 1 because they would skew the results. Similar conclusions regarding the structure of prefactors follow, however, and it is interesting to note the obvious symmetry of multiplicities.

One may wonder why polytope 4309 gives rise to such high multiplicities. Examining the polytope vertices and GLSM charges in table 3, we see that there is a high degree of symmetry in the vertices which give rise to many $\mathbb{P}^{1}$-like GLSM relations. This symmetry likely increases the number of triangulations, and also increases the number of intersection curves $\Sigma \subset B_{3}$ which are $\mathbb{P}^{1}$. Geometrically, this polytope and its associated toric varieties are rather unique in the $d=3$ Kreuzer-Skarke list, and it would be interesting to study them further.

\section{Points of $E_{8}$ and Superpotential Zeroes}

In the example of section 8.2, we will see that the prefactor vanishes if and only if there exists a point of $E_{8}$ enhancement in the worldvolume of the M5-instanton. Before getting to the example, in this section we will explore the connection between points of $E_{8}$ enhancement and prefactor zeroes on more general grounds. Points of $E_{8}$ enhancement are known to give rise to interesting phenomenological features [61, but it is a priori unclear how a compactification would arrive at such a high codimension locus in complex structure moduli space; to date, there is no reason to expect stabilization at this point. We will demonstrate via simple geometric arguments that there is often a correspondence between points of $E_{8}$ enhancement and zeroes of the M5-instanton corrections to the superpotential, which often play an important role in moduli stabilization.

Before noting special features which occur in the geometry in the presence of a 


\begin{tabular}{cccccccccccccc}
$x_{1}$ & $x_{2}$ & $x_{3}$ & $x_{4}$ & $x_{5}$ & $x_{6}$ & $x_{7}$ & $x_{8}$ & $x_{9}$ & $x_{10}$ & $x_{11}$ & $x_{12}$ & $x_{13}$ & $x_{14}$ \\
\hline 1 & 0 & 0 & 0 & 0 & 0 & 0 & 0 & 0 & 0 & 0 & 0 & 0 & 1 \\
0 & 1 & 0 & 0 & 0 & 0 & 0 & 0 & 0 & 0 & 0 & 0 & 1 & 0 \\
0 & 0 & 1 & 0 & 0 & 0 & 0 & 0 & 0 & 0 & 0 & 1 & 0 & 0 \\
0 & 0 & 0 & 1 & 0 & 0 & 0 & 0 & 0 & 0 & 1 & 0 & 0 & 0 \\
0 & 0 & 0 & 0 & 1 & 0 & 0 & 0 & 0 & 1 & 0 & 0 & 0 & 0 \\
0 & 0 & 0 & 0 & 0 & 1 & 0 & 0 & 1 & 0 & 0 & 0 & 0 & 0 \\
0 & 0 & 0 & 0 & 0 & 0 & 1 & 1 & 0 & 0 & 0 & 0 & 0 & 0 \\
0 & 0 & 0 & 0 & 0 & 0 & 0 & 1 & 0 & 0 & 0 & 0 & 1 & -1 \\
0 & 0 & 0 & 0 & 0 & 0 & 0 & 0 & 1 & 0 & 0 & 1 & 0 & -1 \\
0 & 0 & 0 & 0 & 0 & 0 & 0 & 0 & 0 & 1 & 0 & 1 & 1 & -2 \\
0 & 0 & 0 & 0 & 0 & 0 & 0 & 0 & 0 & 0 & 1 & -1 & -1 & 1
\end{tabular}

\begin{tabular}{cccccccccccccc}
$v_{1}$ & $v_{2}$ & $v_{3}$ & $v_{4}$ & $v_{5}$ & $v_{6}$ & $v_{7}$ & $v_{8}$ & $v_{9}$ & $v_{10}$ & $v_{11}$ & $v_{12}$ & $v_{13}$ & $v_{14}$ \\
\hline 1 & 0 & 0 & -1 & 2 & 1 & 1 & -1 & -1 & -2 & 1 & 0 & 0 & -1 \\
0 & 1 & 0 & 1 & -1 & 0 & -1 & 1 & 0 & 1 & -1 & 0 & -1 & 0 \\
0 & 0 & 1 & 1 & -1 & -1 & 0 & 0 & 1 & 1 & -1 & -1 & 0 & 0
\end{tabular}

Table 3: The first table gives the homogeneous coordinates and associated GLSM charges for all toric varieties corresponding to polytope 4309. We do not give the Stanley-Reisner ideal, since this data is determined by the triangulation. The second table gives the point matrix of the polytope. The point matrix exhibits the symmetry $v_{i}+v_{15-i}=0$, trivially giving the first 7 rows of the table of GLSM charges.

point of $E_{8}$ enhancement, let us recall the basic geometric setup. The prefactor is computed by computing the line bundle cohomology describing instanton zero modes on a distinguished spectral curve $c_{l o c}$. In the discussion here, we will consider this curve as as sitting in an elliptic surface $\mathcal{E}$, and thus the discussion here applies to both the F-theory and heterotic cases. The curve $c_{l o c}$ has a defining equation given by (5.16)

$$
f_{c_{l o c}}=\sum_{q=1}^{n} \tilde{b}_{q} v^{n-q} x^{n_{x}} y^{n_{y}}
$$

where $q=2 n_{x}+3 n_{y}$. The sections $\tilde{b}_{q}$ in the defining equations for the spectral curve $c_{l o c}$ are obtained via restricting the sections $b_{q}$ in the defining equation for the spectral cover $\mathcal{C}_{\text {loc }}$ to $\mathcal{E}$. Since $b_{q}$ is a section of $K_{B_{2}}^{\otimes q} \otimes \mathcal{O}_{B_{2}}(\eta)$ for $\eta$ a curve in $B_{2}, \tilde{b}_{q}$ is a section of $\mathcal{O}_{\Sigma}(r-q \chi)$ where $\Sigma$ is a $\mathbb{P}^{1}$ and $\chi \equiv c_{1} \cdot B_{2} \Sigma, r \equiv \eta \cdot B_{2} \Sigma$. We are interested in line bundle cohomology on $c_{l o c}$, which we choose to compute via the Koszul sequence (5.21) from the restriction of a line bundle on $\mathcal{E}$. We write this bundle as $\mathcal{O}_{\mathcal{E}}(a s+b F)$, where $s$ is the section of $\mathcal{E}$ and $F$ is the class of the elliptic fiber.

The special features present in the case of a point of $E_{8}$ enhancement can be seen by considering the intersections of $s$ and $F$ with $c_{l o c}$. Inside $\mathcal{E}, s \cdot c_{l o c}$ is defined by the locus

$$
s \cdot c_{l o c}=\{v=0\} \cdot\left\{f_{c_{l o c}}=0\right\}=\left\{\tilde{b}_{n}=0\right\} .
$$


Since $\tilde{b}_{n}$ is a section of degree $r-n \chi$, it has $r-n \chi$ zeroes $u_{k}$ on $\Sigma=\mathbb{P}^{1}$ and therefore $s \cdot c_{l o c}$ is the collection of points

$$
s \cdot c_{l o c}=\sum_{k=1}^{r-n \chi}\left\{e, u_{k}\right\}
$$

where $e$ is the identity of the elliptic fiber. Similarly, in $\mathcal{E}$ above any point $u \in \Sigma$ there is an elliptic fiber $F_{u}$. The fiber $F_{u}$ above $u$ intersects $c_{l o c}$ at $n$ points $q_{i}$ in the elliptic fiber, so that

$$
F_{u} \cdot c_{l o c}=\sum_{i=1}^{n}\left\{q_{i}, u\right\} .
$$

Intersecting $n$ copies of $s$ with $c_{l o c}$, we obtain

$$
n s \cdot c_{l o c}=\sum_{k=1}^{r-n \chi}\left\{n e, u_{k}\right\}=\left\{n e, u_{j}\right\}+\sum_{k=1, k \neq j}^{r-n \chi}\left\{n e, u_{k}\right\},
$$

where we have separated off a distinguished root $u_{j}$ of $\tilde{b}_{n}$ for convenience. In the case that $u_{j}$ is a point of $E_{8}$ enhancement, $u_{j}$ is a root not only of $\tilde{b}_{n}$ but also of $\tilde{b}_{q}$ from $2 \ldots n-1$. Evaluating the $\tilde{b}_{q}$ 's at $u_{j}$ gives $f_{c_{l o c}}=\tilde{b}_{0} v^{n}$ and therefore $F_{u_{j}} \cdot c_{l o c}=$ $\sum_{i=1}^{n}\left\{e, u_{j}\right\}=\left\{n e, u_{j}\right\}$. Then

$$
\left(n s-F_{u j}\right) \cdot c_{l o c}=\sum_{k=1, k \neq j}^{r-n \chi}\left\{n e, u_{k}\right\}
$$

which means that $\left.\mathcal{O}_{\mathcal{E}}(n s-F)\right|_{c_{l o c}}$ has a section. Summarizing these arguments, in the presence of a point of $E_{8}$ enhancement $\left.\mathcal{O}_{\mathcal{E}}(n s-F)\right|_{c_{l o c}}$ has a section. Of course, in 7.5 we could split off up to $r-n \chi$ points. Similar arguments therefore lead to the conclusion that in the presence of $l$ points of $E_{8}$ enhancement, $\left.\mathcal{O}_{\mathcal{E}}(n s-a F)\right|_{c_{l o c}}$ has a section for $a$ in $1 \ldots l$. For convenience, let us give these bundles a name, $\left.\mathcal{L}_{E 8, a} \equiv \mathcal{O}_{\mathcal{E}}(n s-a F)\right|_{c_{l o c}}$. It is important to note that these bundles typically get a section at high codimension in the bundle moduli space. It is only in the case of $n=3$ bundles corresponding to $G_{4 d}=E_{6}$ where an $\mathcal{L}_{E 8, a}$ bundle gets a section in codimension one.

Having discussed the line bundles $\mathcal{L}_{E 8, a}$ and under what circumstances they obtain a section, let us discuss the relationship to the vanishing of the prefactor in light of the example of section 8.2 . There, by realizing that $A_{\Sigma} \sim f_{E 8}^{4}$ where $f_{E 8}=\operatorname{Res}\left(\tilde{b}_{2}, \tilde{b}_{3}\right)$, it will be shown that the prefactor vanishes if and only if there exists a point of $E_{8}$ enhancement in the worldvolume of the instanton. How does one understand this at the level of line bundles? In this example the prefactor vanishes if and only if the bundle $\mathcal{L}_{A}=\left.\mathcal{O}_{\mathcal{E}}(6 s-F)\right|_{c_{l o c}}$ has a section, which occurs only on the $f_{E 8}=0$ locus in moduli space. On this locus, $\tilde{b}_{2}$ and $\tilde{b}_{3}$ have a common zero which plays the role of $u_{j}$ in the above discussion, and therefore $\mathcal{L}_{E 8,1}=\left.\mathcal{O}_{\mathcal{E}}(3 s-F)\right|_{c_{l o c}}$ has a section, where $n=3$ in this example. An important observation is that

$$
\mathcal{L}_{A}=\left.\mathcal{O}_{\mathcal{E}}(6 s-F)\right|_{c_{l o c}}=\left.\mathcal{O}_{\mathcal{E}}(3 s)\right|_{c_{l o c}} \otimes \mathcal{L}_{E 8,1},
$$

where it is convenient that $\left.\mathcal{O}_{\mathcal{E}}(3 s)\right|_{c_{l o c}}$ always have a section. Since this is true, $\mathcal{L}_{A}$ has a section whenever $\mathcal{L}_{E 8,1}$ has a section, simply by multiplication of the sections of $\mathcal{L}_{E 8,1}$ 
and $\left.\mathcal{O}_{\mathcal{E}}(3 s)\right|_{c_{l o c}}$. In this special $n=3$ case, where $\mathcal{L}_{E 8,1}$ has a section in codimension one on $f_{E 8}=0$, this can and does (in this example) govern the entire prefactor. For higher $n$ cases, such as the $n=5$ case with $G_{4 d}=S U(5)$, vanishing loci triggered by points of $E_{8}$ enhancement typically sit as a proper sublocus in the codimension one vanishing locus of the prefactor.

Using these ideas, let us discuss a generalization of the circumstances under which points of $E_{8}$ in the M5-instanton worldvolume will give rise to a prefactor zero. Recall from (5.20) that

$$
\mathcal{L}_{A}=\left.\left.\mathcal{O}_{\mathcal{E}}\left(n\left(\frac{1}{2}+\lambda\right) s+\left(r\left(\frac{1}{2}-\lambda\right)+\chi\left(\frac{1}{2}+n \lambda\right)-1\right) F\right)\right|_{c_{l o c}} \equiv \mathcal{O}_{\mathcal{E}}\left(n\left(\frac{1}{2}+\lambda\right) s+B F\right)\right|_{c_{l o c}}
$$

where we have defined $B$ for convenience and $\lambda$ is half-integral (integral) for $n$ odd (even). Let us examine the conditions under which $l$ points of $E_{8}$ in the instanton worldvolume will cause the prefactor to vanish. Splitting the bundle as above, but generalizing the result, we have

$$
\mathcal{L}_{A}=\left.\mathcal{O}_{\mathcal{E}}\left(n\left(\lambda-\frac{1}{2}\right) s+(B+l) F\right)\right|_{\text {loc }} \otimes \mathcal{L}_{E 8, l} .
$$

In the presence of $l$ points of $E_{8}$ in the instanton worldvolume, $\mathcal{L}_{E 8, l}$ has a section, and therefore the prefactor vanishes if the first bundle in the product has a section. For simplicity we restrict attention to the case of $n$ odd, since the $n=3$ case is the case of the examples we study and $n=5$ is the case relevant for four-dimensional $S U(5) \mathrm{GUTs}$. If $\lambda=\frac{1}{2}$ the first factor is simply $\left.\mathcal{O}_{\mathcal{E}}((B+l) F)\right|_{c_{l o c}}$ which has a section if $B+l \geq 0$. In the case of $\lambda>\frac{1}{2}$, the first bundle in the product takes the form $\left.\left.\mathcal{O}_{\mathcal{E}}(P s)\right|_{c_{l o c}} \otimes \mathcal{O}_{\mathcal{E}}(n s+(B+l) F)\right|_{c_{l o c}}$ where $P \equiv n\left(\lambda-\frac{1}{2}\right)-n \geq 0$, and we can restrict our attention to $\left.\mathcal{O}_{\mathcal{E}}(n s+(B+l) F)\right|_{c_{l o c}}$ since $\left.\mathcal{O}_{\mathcal{E}}(P s)\right|_{c_{l o c}}$ has a section. For $(B+l) \geq 0$ this bundle has a section and for $(B+l)<0$ it is simply $\mathcal{L}_{E 8, B+l}$, which has a section for $B+l \geq-l$, since we have $l$ points of $E_{8}$ present in the instanton worldvolume.

Summarizing the results for $n$ odd, covering $E_{6}$ and $S U(5)$ GUTs, we have that $l$ points of $E_{8}$ will cause a prefactor zero if

$$
\begin{array}{ll}
\lambda=\frac{1}{2}: & B+l \geq 0 \\
\lambda>\frac{1}{2}: & B+l \geq-l \Rightarrow B+2 l \geq 0 .
\end{array}
$$

In every example $B$ can be computed explicitly and these conditions can be checked. This is useful because it is computationally intensive to explicitly compute a generic prefactor, but these conditions allow one to determine cases where an $E_{8}$ point causes a prefactor zero without performing the computation. We see that, though the locus of point(s) of $E_{8}$ is typically of high codimension in moduli space, it often sits inside the vanishing locus of the prefactor. Also, while we have only addressed zeroes of the prefactor, we remind the reader that the prefactor often vanishes to high degree and thus it is likely that superpotential zeroes associated with points of $E_{8}$ are also critical points.

As a nontrivial check, let us apply our conditions to the example of section 8.1, which is not the example obviously related to points of $E_{8}$. There the relevant data is

$$
\mathcal{L}_{A}=\left.\mathcal{O}_{\mathcal{E}}(6 s-3 F)\right|_{c_{l o c}} \quad \lambda=\frac{3}{2}
$$


so that $B=-3$. It is easy to see that $l=1$ does not satisfy the condition 7.10 and therefore the prefactor does not vanish in the presence of a single point of $E_{8}$ enhancement. This is reflected by the explicit computation: a point of $E_{8}$ enhancement occurs in codimension one in moduli space at $\operatorname{Res}\left(\tilde{b}_{2}, \tilde{b}_{3}\right)=0$ since $n=3$, and this locus is not the prefactor locus in this example. On the other hand, the conditions (7.10) are satisfied for $l=2$, so that 2 points of $E_{8}$ in the instanton worldvolume cause the prefactor to vanish. Since $\tilde{b}_{2}, \tilde{b}_{3} \in \Gamma\left(\mathcal{O}_{\Sigma}(2)\right)$ in the example, having two points of $E_{8}$ requires that $\tilde{b}_{2}$ and $\tilde{b}_{3}$ are proportional to one another, which requires $\beta_{i} \sim \gamma_{i}$, a condition which clearly causes the prefactor (8.13) to vanish. In this example, $r-n \chi=2$, so two points of $E_{8}$ is the maximal number.

Let us make one final comment in this section about points of $E_{8}$. It was shown from the study of heterotic worldsheet instantons [63] that for $\chi=1$ there is a high codimension locus in moduli space, called $\mathcal{R}$, which always sits inside the vanishing locus of the prefactor. In the F-theory dual, this is caused by the presence of $r-n$ points of $E_{8}$. Though this is for the $\chi=1$ case, we expect that a simple analysis along the lines of [63] would extend it to the $r-n \chi$ case, which is the maximal number of points of $E_{8}$ which could appear in the instanton worldvolume for geometries of this type. As mentioned, the zero caused by two points of $E_{8}$ enhancement in the example of section 8.1 is the maximal number, and so this point in moduli space is in the $\mathcal{R}$ locus.

\section{Illustrative Examples}

In this section we will supplement the discussions of previous sections with explicit examples which demonstrate the various ideas we have discussed. In studying these examples, it will become clear that the instanton prefactor $A$ often factorizes nicely into powers of simpler polynomials. Zeroes of these simpler polynomials often correspond to interesting seven-brane physics.

We will present three examples. The first example is the F-theory dual of one of the heterotic compactifications in [69]. It is an $E_{6}$ GUT realized in a Calabi-Yau fourfold with a base $B_{3}$ which is a generalized Hirzebruch variety. We consider an instanton correction due to a euclidean D3-instanton, and in this example the instanton prefactor is the fourth power of a polynomial $f_{\Lambda}$ which is cubic in moduli. The line bundle $\mathcal{L}_{\Lambda}$ of section 2 obtains a section when $f_{\Lambda}=0$. Thus, in this example we have that $\mathcal{L}_{A}$ obtains a section if and only if $\mathcal{L}_{\Lambda}$ obtains a section.

In the second example, we consider an F-theory compactification with $G_{4 d}=E_{6}$ which does not have a heterotic dual. The base threefold $B_{3}$ can be obtained by blowing up $\mathbb{P}^{3}$ twice along $\mathbb{P}^{1}$ s, and this procedure is discussed in detail in appendix $\mathbb{B}$. For convenience, we will use the description of $B_{3}$ as a toric variety. We will show that a superpotential correction due to a euclidean D3-instanton along a divisor in $B_{3}$ has a prefactor which vanishes if and only if there exists a point of $E_{8}$ enhancement in the worldvolume of the instanton. This exemplifies the discussion of section 7.

In the third example, we consider another F-theory compactification on the same base $B_{3}$ as the second example, but with the $E_{6}$ GUT stack localized on another divisor. In this compactification we compute a new instanton prefactor, again without a heterotic dual, and find that the prefactor is identically zero. 


\subsection{Example One}

In this example we study the prefactor of an M5-instanton correction which was originally computed in the dual heterotic picture in [69], where it was called example 4. It was also discussed from the heterotic point of view in [62] and from the F-theory point of view in 53 .

The global F-theory compactification we consider is an elliptically fibered Calabi-Yau fourfold over a based $B_{3}$ specified by the toric data

\begin{tabular}{cccccc|c}
$x_{0}$ & $x_{1}$ & $x_{2}$ & $x_{3}$ & $z$ & $x_{5}$ & class \\
\hline 1 & 1 & 2 & 0 & 0 & 2 & $A$ \\
0 & 0 & 1 & 1 & 0 & 2 & $S$ \\
0 & 0 & 0 & 0 & 1 & 1 & $B_{2}$ \\
\multicolumn{5}{c}{$S R I=\left\langle x_{0} x_{1}, x_{2} x_{3}, z x_{5}\right\rangle}$,
\end{tabular}

where we have specified the GLSM charges of the homogeneous coordinates and also the Stanley-Reisner ideal. We have defined the divisor classes $A \equiv\left[x_{1}=0\right], S \equiv\left[x_{3}=0\right]$, and $B_{2} \equiv[z=0]$. In this basis the non-zero intersection numbers are

$$
B_{2} A S=1 \quad B_{2}^{2} A=-2 \quad B_{2}^{2} S=2 \quad B_{2} S^{2}=-2
$$

We see that

$$
c_{1}\left(B_{3}\right)=6 A+4 S+2 B_{2}
$$

and the defining equation for the Calabi-Yau with an $E_{6}$ GUT stack on $B_{2} \equiv\{z=0\}$ in $B_{3}$ is given by

$$
y^{2}=x^{3}+f x z^{4} v^{4}+g z^{6} v^{6}+z^{2} v^{4}\left(b_{0} z^{3} v^{3}+b_{2} z v x+b_{3} y\right)
$$

where the objects appearing in this equations are sections of the bundles

\begin{tabular}{c|c} 
Section & Bundle \\
\hline$x_{4}$ & $\mathcal{O}\left(B_{2}\right)$ \\
$b_{m}$ & $\mathcal{O}\left([6-m] c_{1}\left(B_{3}\right)-[5-m] B_{2}\right)$ \\
$f$ & $\mathcal{O}\left(c_{1}\left(B_{3}\right)-B_{2}\right)^{\otimes 4}$ \\
$g$ & $\mathcal{O}\left(c_{1}\left(B_{3}\right)-B_{2}\right)^{\otimes 6}$.
\end{tabular}

The GUT stack $B_{2}$ is the second Hirzebruch surface $\mathbb{F}_{2}$ and the heterotic dual to this F-theory compactification is an $E_{8} \times E_{8}$ compactification on a Calabi-Yau threefold which is elliptically fibered over $B_{2}$ and with bundle data appropriately mapped. It is worth noting that $B_{2} \cdot S$ is the curve $\Sigma$ in $\mathbb{F}_{2}$ with $\Sigma^{2}=-2$. This curve is the curve $\Sigma$ relevant for the instanton physics we will discuss. For convenience throughout we will define line bundles on $B_{3}$ as

$$
\mathcal{O}(a, b, c) \equiv \mathcal{O}\left(a A+b S+c B_{2}\right) .
$$

Since they play an important role in determining the instanton physics, let us discuss the sections $b_{0}, b_{2}$ and $b_{3}$ more explicitly. In this example, $b_{m} \in H^{0}\left(B_{3}, \mathcal{O}((6-m) 6,(6-\right.$ $m) 4,7-m)$ ), so that

$$
b_{0} \in H^{0}\left(B_{3}, \mathcal{O}(36,24,7)\right)
$$




$$
\begin{aligned}
& b_{2} \in H^{0}\left(B_{3}, \mathcal{O}(24,16,5)\right) \\
& b_{3} \in H^{0}\left(B_{3}, \mathcal{O}(18,12,4)\right) .
\end{aligned}
$$

It is clear that such non-trivial sections exist, and therefore an $E_{6}$ GUT on $B_{2}$ can in fact be realized in this geometry. These sections determine the moduli dependence of the $E_{6}$ GUT. They also also determine the compact Higgs bundle spectral cover $\mathcal{C}_{\text {loc }}$ as in (3.16). For convenience, let us note that

$$
\eta=\left.\left(36 A+24 S+7 B_{2}\right)\right|_{B_{2}} \quad \text { and } \quad c_{1, B_{2}}=\left.\left(6 A+4 S+B_{2}\right)\right|_{B_{2}}
$$

using adjunction and the definitions in 5.2.

Let us turn to a discussion of the instanton physics. Consider an M5-instanton wrapped on $\pi^{-1} D 3$ where $D 3 \equiv D_{3} \equiv\left\{x_{3}=0\right\}$ inside $B_{3}$. It turns out that this instanton does not actually contribute to the superpotential, since $h^{i}\left(D 3, N_{D 3 \mid B_{3}}\right)=$ $(0,1,0)$, violating the necessary condition (3.27). In the dual heterotic picture where this example was first studied, the non-contribution to the superpotential is related to the fact that [69] focused on left-moving zero modes which couple to the gauge bundle and not the right-moving zero modes which do not. The study of left-movers leads to knowledge of the prefactor, whereas the spectrum of right-movers determines whether the instanton contributes to the superpotential or some other holomorphic coupling. In the language of type IIb, this instanton does not have the right spectrum of uncharged $3-3$ zero modes for a superpotential correction.

To determine the component of the prefactor $A$ which depends on seven-brane moduli, we need to study instanton zero modes localized on the curve $\Sigma=B_{2} \cap D 3$ in $B_{3}$ where the instanton intersects the GUT stack. Topologically, $\Sigma$ is a $\mathbb{P}^{1}$. As discussed in section 5, rather than computing vector bundle cohomology on $\Sigma$ we compute an isomorphic line bundle cohomology on a spectral curve $c_{l o c}$ which is a triple-sheeted cover of $\Sigma$. Following section [5.2, we compute directly that $r=B_{2} \cdot S \cdot \eta=2$ and $\chi=B_{2} \cdot S \cdot c_{1, B_{2}}=0$ and choose $n=3$ (for an $E_{6}$ GUT) and $\lambda=\frac{3}{2}$. Using (5.15) and (5.20), this gives the class of $c_{l o c}$ as a divisor inside an elliptic surface $\mathcal{E}$ and the relevant line bundle $\mathcal{L}_{A}$ for computing the prefactor to be

$$
\left[c_{\text {loc }}\right]=3 s+2 F \quad \mathcal{L}_{A}=\mathcal{O}_{\mathcal{E}}(6 s-3 F) .
$$

In terms of the spectral cover, $c_{l o c}=\left.\mathcal{C}_{l o c}\right|_{\pi^{-1} D 3}$. At the level of defining equations, this means that $f_{c_{l o c}}=\left.f_{\mathcal{C}_{l o c}}\right|_{x_{3}=0}$, and therefore

$$
f_{c_{l o c}}=\tilde{b}_{0} v^{3}+\tilde{b}_{2} v x+\tilde{b}_{3} y
$$

where $\left.\tilde{b}_{m} \equiv b_{m}\right|_{G U T \cap D 3}=\left.b_{m}\right|_{z=x_{3}=0}$. Remembering that $\mathcal{C}_{\text {loc }}$ is naturally a divisor in an ambient elliptic threefold $Z_{3, F}$, $c_{l o c}$ is therefore a divisor in an ambient elliptic surface $\mathcal{E}=\pi_{3, F}^{-1} \Sigma$ given by the restriction of the elliptic threefolds to $x_{3}=0$. While it initially seems strange to be considering these ambient spaces which do not sit inside the Calabi-Yau fourfold, the physics we study is determined entirely by line bundle cohomology on $c_{l o c}$, which does sit inside the Calabi-Yau. One is free to compute line bundle cohomology on $c_{l o c}$ via any allowed means, including via a Koszul sequence from the ambient space $\mathcal{E}$ which is an elliptic fibration over $\Sigma$. 
Let us say more about the structure of $f_{c_{l o c}}$ by studying the sections $\tilde{b}_{m}$. Given $b_{m}$ as in (8.7), the monomials in $b_{m}$ with neither a $z$ nor an $x_{3}$ are the ones appearing in $\tilde{b}_{m}$ and take the form

$$
\begin{array}{rlrl}
\tilde{b}_{0} \text { monomials } & \sim x_{5}^{7} x_{2}^{10} x_{0}^{i} x_{1}^{2-i} & i=0 \ldots 2 \\
\tilde{b}_{2} \text { monomials } \sim x_{5}^{5} x_{2}^{6} x_{0}^{i} x_{1}^{2-i} & i=0 \ldots 2 \\
\tilde{b}_{3} \text { monomials } \sim x_{5}^{4} x_{2}^{4} x_{0}^{i} x_{1}^{2-i} & i=0 \ldots 2
\end{array}
$$

where the range of $i$ ensures that the monomials are global sections. From the StanleyReisner ideal, it can be seen that $x_{5}$ and $x_{3}$ must be non-zero since $z=x_{2}=0$. Using two of the scaling relations of the toric variety to set $x_{5}=x_{3}=1$, we can write down unambiguously

$$
\begin{aligned}
& \tilde{b}_{0}=\alpha_{1} x_{0}^{2}+\alpha_{2} x_{0} x_{1}+\alpha_{3} x_{1}^{2} \\
& \tilde{b}_{2}=\beta_{1} x_{0}^{2}+\beta_{2} x_{0} x_{1}+\beta_{3} x_{1}^{2} \\
& \tilde{b}_{3}=\gamma_{1} x_{0}^{2}+\gamma_{2} x_{0} x_{1}+\gamma_{3} x_{1}^{2}
\end{aligned}
$$

in terms of moduli $\alpha_{j}, \beta_{j}$, and $\gamma_{k}$ and the homogeneous coordinates $\left(x_{0}, x_{1}\right)$ on $\Sigma$. While direct computation of $\chi(\Sigma)=2$ shows that it is a $\mathbb{P}^{1}$, it is also fairly easy to see this from looking at the GLSM charges and Stanley-Reisner ideal in (8.1).

We have calculated the quantities $\{n, \lambda, r, \chi\}$ as outlined in section [5.2, and written the defining equation $f_{c_{l o c}}$. Given this data, it is possible to calculate the prefactor of an M5-instanton on $\pi^{-1} D 3$ via a long exact sequence in cohomology involving $h^{i}\left(c_{l o c},\left.\mathcal{L}_{A}\right|_{c_{l o c}}\right)$. For this example, the computation was performed in the dual heterotic picture [69] and was discussed in F-theory [53. To avoid cluttering an already detailed story, we simply quote the result and refer the reader to the literature for an explicit demonstration of the computation. The prefactor is given by

$$
A \sim\left(\epsilon_{i j k} \alpha_{i} \beta_{j} \gamma_{k}\right)^{4} \equiv f_{\Lambda}^{4}
$$

where $f_{\Lambda}$ is defined for convenience. A number of questions are valid at this point: why does the instanton prefactor, a degree twelve polynomial, factorize so nicely? What is the significance of the polynomial $f_{\Lambda}$ and of the power to which it is raised?

\subsubsection{Flat bundles, Intermediate Jacobians, and the Prefactor}

In this subsection we will review the significance of the polynomial $f_{\Lambda}$, which determines the vanishing locus of the instanton prefactor in this example, and its relationship with the theta divisor on the intermediate Jacobian of the M5 instanton.

Let us begin by reviewing the significance of the polynomial $f_{\Lambda}$, as discussed in the heterotic case in [62]. A brief look at $f_{\Lambda}$ reveals that is it

$$
f_{\Lambda}=\operatorname{det}\left(\begin{array}{ccc}
\alpha_{1} & \alpha_{2} & \alpha_{3} \\
\beta_{1} & \beta_{2} & \beta_{3} \\
\gamma_{1} & \gamma_{2} & \gamma_{3}
\end{array}\right) \equiv \operatorname{det} M
$$

it is natural to ask the significance of this matrix $M$. If one were to compute the line bundle cohomology of $\left.\mathcal{O}_{\mathcal{E}}(3 s-2 F)\right|_{c_{l o c}}$ via the Koszul sequence

$$
\left.0 \rightarrow \mathcal{O}_{\mathcal{E}}(-4 F) \stackrel{f_{c_{l o c}}}{\longrightarrow} \mathcal{O}_{\mathcal{E}}(3 s-2 F) \rightarrow \mathcal{O}_{\mathcal{E}}(3 s-2 F)\right|_{c_{l o c}} \rightarrow 0
$$


and the corresponding long exact sequence in cohomology, one would find that $M$ appears as the matrix map between $H^{1}\left(\mathcal{E}, \mathcal{O}_{\mathcal{E}}(-4 F)\right)$ and $H^{1}\left(\mathcal{E}, \mathcal{O}_{\mathcal{E}}(3 s-2 F)\right)$. Defining $\left.\Lambda \equiv \mathcal{O}_{\mathcal{E}}(3 s-2 F)\right|_{c_{l o c}}$, the statement then is that $f_{\Lambda}=0$ is the codimension 1 locus in moduli space on which $\Lambda$ obtains a section. Therefore, by comparing to equation (8.13) we see that the prefactor vanishes if and only if $\Lambda$ obtains a section. What governs the relationship between the bundle $\Lambda$ and the bundle $\mathcal{L}_{A}$ which actually determines the prefactor? Decomposing as

$$
\left.\mathcal{L}_{A} \equiv \mathcal{O}_{\mathcal{E}}(6 s-3 F)\right|_{c_{l o c}}=\left.\mathcal{O}(F)\right|_{c_{l o c}} \otimes \Lambda^{\otimes 2} \equiv \mathcal{L}_{1} \otimes \Lambda^{\otimes 2}
$$

will be useful for demonstrating the relationship. On the locus $f_{\Lambda}=0, \Lambda$ has a section and therefore $\Lambda^{\otimes 2}$ also has a section. But $\mathcal{L}_{1}$ itself has a section for all moduli, and therefore when $f_{\Lambda}=0, \mathcal{L}_{A}$ also has a section and the prefactor vanishes. Thus, we see that the polynomial substructure of the prefactor in this example actually corresponds to the locus in moduli space where a related bundle $\Lambda$ obtains a section.

Let us state how this is related to the discussion in previous sections. The bundle $\Lambda$ is a degree 0 bundle and therefore specifies a point in $\mathcal{J}_{0}\left(c_{l o c}\right) . \mathcal{L}_{A}$, the bundle directly related to the prefactor, is a degree $g_{c_{l o c}}-1$ bundle but can be decomposed as

$$
\mathcal{L}_{A}=K_{c_{l o c}}^{\frac{1}{2}} \otimes \mathcal{L}_{\Gamma}=K_{c_{l o c}}^{\frac{1}{2}} \otimes \Lambda^{\otimes \lambda} .
$$

The spin structure defines a map from $\mathcal{J}_{0}\left(c_{l o c}\right) \leftrightarrow \mathcal{J}_{g-1}\left(c_{\text {loc }}\right)$ via tensor product. In general, when $\mathcal{L}_{A}$ has a section, it corresponds to a point in the Riemann theta divisor $\Theta_{R}$ in $\mathcal{J}_{g-1}\left(c_{l o c}\right)$. A choice of spin structure maps $\Theta_{R}$ to a particular theta divisor $\Theta_{m}$ on $\mathcal{J}_{0}\left(c_{l o c}\right)$, which in general does not have anything to do with degree 0 bundles which have a section. However, in this case it does and that bundle is $\Lambda$.

Finally, let us make a speculative comment about the multiplicity of $f_{\Lambda}$, namely that $A \sim f_{\Lambda}^{4}$. Physically, one should expect that the order of vanishing is related to the number of zero modes. A single zero mode would be enough to cause a prefactor zero, but in the presence of many modes any will cause a zero, and thus the prefactor zero should be of high order. This makes sense from a type IIb point of view, where it is known that increasing the flux quanta analogous to $\lambda$ increases the number of $D 3-D 7$ zero modes and also the order of vanishing of the instanton prefactor. In this example, the Hodge numbers are $h^{i}\left(c_{l o c}, \mathcal{L}_{A}\right)=(2,2)$, and thus there are four zero modes. It is natural and tempting to think that this is related to the fourth power of $f_{\Lambda}$ in the prefactor, but one would need to study these ideas further to put them on firmer footing.

\subsection{Example Two: Prefactors without a heterotic dual}

In this section we study instantons in a global F-theory compactification which does not admit a heterotic dual. Interestingly, the prefactor computed here in an intrinsically F-theoretic geometry is mathematically identical to a prefactor computation in the heterotic string in [59, despite being in a different compactification. This exemplifies the multiplicities seen in section 6. Some of the prefactor structure was addressed in [62], though we will be focused on the F-theory physics here. We will also discuss other aspects of the geometry and example. 
The global F-theory compactification we consider is an elliptically fibered Calabi-Yau fourfold over a based $B_{3}$ specified by the toric data

\begin{tabular}{rccccc|c}
$x_{0}$ & $x_{1}$ & $x_{2}$ & $x_{3}$ & $z$ & $x_{5}$ & class \\
\hline 1 & 0 & 0 & 1 & 1 & 1 & $H$ \\
0 & 1 & 0 & -1 & 0 & -1 & $E_{1}$ \\
0 & 0 & 1 & 0 & -1 & -1 & $E_{2}$ \\
$S R I=\left\langle x_{2} x_{3}, x_{3} x_{5}, z x_{5}, x_{0} x_{1} x_{2}, x_{0} x_{1} z\right\rangle$,
\end{tabular}

where we have specified the GLSM charges of the homogeneous coordinates and also the Stanley-Reisner ideal. While this parameterization is most useful for comparing to the non-toric analysis of appendix $\mathrm{B}$, we now choose a different parameterization of the same toric variety. Adding the first row to the second and third rows, we have

\begin{tabular}{rccccc|c}
$x_{0}$ & $x_{1}$ & $x_{2}$ & $x_{3}$ & $z$ & $x_{5}$ & class \\
\hline 1 & 0 & 0 & 1 & 1 & 1 & $X$ \\
1 & 1 & 0 & 0 & 1 & 0 & $E_{1}$ \\
1 & 0 & 1 & 1 & 0 & 0 & $E_{2}$ \\
$S R I=\left\langle x_{2} x_{3}, x_{3} x_{5}, z x_{5}, x_{0} x_{1} x_{2}, x_{0} x_{1} z\right\rangle$,
\end{tabular}

where $X \equiv H-E_{1}-E_{2}$. In the basis $\left\{X, E_{1}, E_{2}\right\}$, the intersection numbers are

$$
\begin{aligned}
& X X X=1 \quad X X E_{1}=-1 \quad X X E_{2}=-1 \quad X E_{1} E_{1}=1 \\
& X E_{1} E_{2}=1 \quad X E_{2} E_{2}=1 \quad E_{1} E_{1} E_{1}=-2 \quad E_{1} E_{1} E_{2}=0 \\
& E_{1} E_{2} E_{2}=-1 \quad E_{2} E_{2} E_{2}=-1 \text {. }
\end{aligned}
$$

We see that

$$
c_{1}\left(B_{3}\right)=4 X+3 E_{1}+3 E_{2}
$$

and the defining equation for the Calabi-Yau with an $E_{6}$ GUT stack on $B_{2} \equiv\{z=0\}$ in $B_{3}$ is given by

$$
y^{2}=x^{3}+f x z^{4} v^{4}+g z^{6} v^{6}+z^{2} v^{4}\left(b_{0} z^{3} v^{3}+b_{2} z v x+b_{3} y\right)
$$

where the objects appearing in this equations are sections of the bundles

\begin{tabular}{c|c} 
Section & Bundle \\
\hline$x_{4}$ & $\mathcal{O}\left(B_{2}\right)$ \\
$b_{m}$ & $\mathcal{O}\left([6-m] c_{1}\left(B_{3}\right)-[5-m] B_{2}\right)$ \\
$f$ & $\mathcal{O}\left(c_{1}\left(B_{3}\right)-B_{2}\right)^{\otimes 4}$ \\
$g$ & $\mathcal{O}\left(c_{1}\left(B_{3}\right)-B_{2}\right)^{\otimes 6}$.
\end{tabular}

The GUT stack satisfies $\int_{B_{2}} c_{1}^{2}=8$ and $\int_{B_{2}} c_{2}=4$ which is consistent with $B_{2}$ being the first Hirzebruch surface $\mathbb{F}_{1}$. For convenience throughout we will define line bundles on $B_{3}$ as

$$
\mathcal{O}(a, b, c) \equiv \mathcal{O}\left(a X+b E_{1}+c E_{2}\right) .
$$

Since they play an important role in determining the instanton physics, let us discuss the sections $b_{0}, b_{2}$ and $b_{3}$ more explicitly. In this example, $b_{m} \in H^{0}\left(B_{3}, \mathcal{O}(19-3 m, 13-\right.$ $2 m, 18-3 m))$, so that

$$
b_{0} \in H^{0}\left(B_{3}, \mathcal{O}(19,13,18)\right)
$$




$$
\begin{aligned}
& b_{2} \in H^{0}\left(B_{3}, \mathcal{O}(13,9,12)\right) \\
& b_{3} \in H^{0}\left(B_{3}, \mathcal{O}(10,7,9)\right) .
\end{aligned}
$$

It is clear that such non-trivial sections exist, and therefore an $E_{6}$ GUT on $B_{2}$ can in fact be realized in this geometry. These sections determine the entire moduli dependence of the $E_{6}$ GUT. They also also determine the compact Higgs bundle spectral cover $\mathcal{C}_{\text {loc }}$ as in (3.16). For convenience, note that

$$
\eta=\left.\left(19 X+13 E_{1}+18 E_{2}\right)\right|_{B_{2}} \quad \text { and } \quad c_{1, B_{2}}=\left.\left(3 X+2 E_{1}+3 E_{2}\right)\right|_{B_{2}}
$$

in this compactification.

Let us turn to a discussion of the instanton physics. Consider an M5-instanton wrapped on $\pi^{-1} D 3$ where $D 3 \equiv D_{1} \equiv\left\{x_{1}=0\right\}$ inside $B_{3}$. As discussed in section 3 , obtaining a superpotential correction requires $\chi\left(D 3, N_{D 3 \mid B_{3}}\right)=0$, and for this instanton it is satisfied, since $h^{i}\left(D, N_{D 3 \mid B_{3}}\right)=(0,0,0)$. In the language of M5-instantons, these constraints are related to the arithmetic genus constraint.

To determine the component of the Pfaffian prefactor $A$ which depends on sevenbrane moduli, we need to study instanton zero modes localized on the curve $\Sigma=B_{2} \cap D 3$ in $B_{3}$ where the instanton intersects the GUT stack. Topologically, $\Sigma$ is a $\mathbb{P}^{1}$. As discussed in section [5.1 rather than computing vector bundle cohomology on $\Sigma$ we compute an isomorphic line bundle cohomology on a spectral curve $c_{l o c}$ which is a triplesheeted cover of $\Sigma$. Following section 5.2, we compute directly that $r=\eta \cdot B_{2} \Sigma=5$ and $\chi=c_{1, B_{2}} \cdot B_{2} \Sigma=1$ and we have $n=3$ (for an $E_{6}$ GUT) and choose $\lambda=\frac{3}{2}$. From equations (5.15) and (5.20), this gives the class of $c_{l o c}$ as a divisor inside an elliptic surface $\mathcal{E}$ and the relevant line bundle $\mathcal{L}_{A}$ for computing the prefactor to be

$$
\left[c_{l o c}\right]=3 s+5 F \quad \mathcal{L}_{A}=\left.\mathcal{O}_{\mathcal{E}}(6 s-F)\right|_{c_{l o c}} .
$$

In terms of the spectral cover, $c_{l o c}=\left.\mathcal{C}_{l o c}\right|_{\pi^{-1} D 3}$. At the level of defining equations, this means that $f_{c_{l o c}}=\left.f_{\mathcal{C}_{l o c}}\right|_{x_{1}=0}$, and therefore

$$
f_{c_{l o c}}=\tilde{b}_{0} v^{3}+\tilde{b}_{2} v x+\tilde{b}_{3} y
$$

where $\left.\tilde{b}_{m} \equiv b_{m}\right|_{G U T \cap D 3}=\left.b_{m}\right|_{z=x_{1}=0}$. Remembering that $\mathcal{C}_{l o c}$ is naturally a divisor in an ambient elliptic threefold $Z_{3, F}, c_{l o c}$ is therefore a divisor in an ambient elliptic surface $\mathcal{E}$ given by the restriction of the elliptic threefolds to $x_{1}=0$. While it initially seems strange to be considering these ambient spaces which do not sit inside the Calabi-Yau fourfold, the physics we study is determined entirely by line bundle cohomology on $c_{l o c}$, which does sit inside the Calabi-Yau. One is free to compute line bundle cohomology on $c_{l o c}$ via any allowed means, including via a Koszul sequence from the ambient space $\mathcal{E}$ which is an elliptic fibration over $\Sigma$.

Let us say more about the structure of $f_{c_{l o c}}$ by studying the sections $\tilde{b}_{m}$. Given $b_{m}$ as in (8.25), the monomials in $b_{m}$ with neither a $z$ nor an $x_{1}$ are the ones appearing in $\tilde{b}_{m}$ and take the form

$$
\begin{array}{ll}
\tilde{b}_{0} \text { monomials } \sim x_{0}^{13} x_{3}^{j} x_{5}^{6-j} x_{2}^{5-j} & j=0 \ldots 5 \\
\tilde{b}_{2} \text { monomials } \sim x_{0}^{9} x_{3}^{j} x_{5}^{4-j} x_{2}^{3-j} & j=0 \ldots 3 \\
\tilde{b}_{3} \text { monomials } \sim x_{0}^{7} x_{3}^{j} x_{5}^{3-j} x_{2}^{2-j} & j=0 \ldots 2
\end{array}
$$


where the range of $j$ has been chosen to ensure that the monomials are global sections. From the Stanley-Reisner ideal, it can be seen that $x_{0}$ and $x_{5}$ must be non-zero since $z=x_{1}=0$. Using two of the scaling relations of the toric variety to set $x_{0}=x_{5}=1$, we can write down unambiguously

$$
\begin{aligned}
& \tilde{b}_{0}=\psi_{1} x_{3}^{5}+\psi_{2} x_{3}^{4} x_{2}^{1}+\psi_{3} x_{3}^{3} x_{2}^{2}+\psi_{4} x_{3}^{2} x_{2}^{3}+\psi_{5} x_{3}^{1} x_{2}^{4}+\psi_{6} x_{2}^{5} \\
& \tilde{b}_{2}=\phi_{1} x_{3}^{3}+\phi_{2} x_{3}^{2} x_{2}^{1}+\phi_{3} x_{3}^{1} x_{2}^{2}+\phi_{4} x_{2}^{3} \\
& \tilde{b}_{3}=\chi_{1} x_{3}^{2}+\chi_{2} x_{3}^{1} x_{2}^{1}+\chi_{3} x_{2}^{2}
\end{aligned}
$$

in terms of moduli $\psi_{j}, \phi_{j}$, and $\chi_{k}$ and the homogeneous coordinates $\left(x_{2}, x_{3}\right)$ on $\Sigma$. While direct computation of $\chi(\Sigma)=2$ shows that it is a $\mathbb{P}^{1}$, it is also fairly easy to see this from looking at the GLSM charges and Stanley-Reisner ideal in (8.19).

We have discussed the moduli dependence of $c_{l o c}$ and also the bundle cohomology which one must compute on it. From this data, it is possible to directly compute the instanton prefactor. Interestingly, though this is an F-theory compactification without a heterotic dual the same topological data $\{n, \lambda, r, \chi\}=\left\{3, \frac{3}{2}, 5,1\right\}$ related to the prefactor appears in a heterotic prefactor computation. The prefactor is given by [59]

$$
\begin{aligned}
& A \sim f_{E 8}{ }^{4} \equiv\left(\chi_{1}^{2} \chi_{3} \phi_{3}^{2}-\chi_{1}^{2} \chi_{2} \phi_{3} \phi_{4}-2 \chi_{1} \chi_{3}^{2} \phi_{3} \phi_{1}-\right. \\
& \chi_{1} \chi_{2} \chi_{3} \phi_{3} \phi_{2}+\chi_{2}^{2} \chi_{3} \phi_{1} \phi_{3}+\phi_{4}^{2} \chi_{1}^{3}- \\
& 2 \phi_{2} \phi_{4} \chi_{3} \chi_{1}^{2}+\chi_{1} \chi_{3}^{2} \phi_{2}^{2}+3 \phi_{1} \phi_{4} \chi_{1} \chi_{2} \chi_{3}+ \\
& \left.\phi_{2} \chi_{1} \phi_{4} \chi_{2}^{2}+\phi_{1}^{2} \chi_{3}^{3}-\phi_{2} \chi_{2} \phi_{1} \chi_{3}^{2}-\phi_{4} \phi_{1} \chi_{2}^{3}\right)^{4}
\end{aligned}
$$

and we again see that this very complicated expression factors into powers of a slightly less complicated polynomial $f_{E 8}$. We will demonstrate that the vanishing associated with $f_{E 8}$ admits a simple physical interpretation. Note also that the moduli $\psi_{i}$ are conspicuously absent the prefactor.

\subsubsection{Points of $E_{8}$ and the Vanishing of the Prefactor}

As in the first example, we see that the prefactor, which is a generically a complicated polynomial in algebraic moduli, factorizes into powers of significantly less complicated polynomials. In this example, we call this polynomial $f_{E 8}$ and would like to discuss its physical significance. This polynomial is independent of the moduli which control the structure of $\tilde{b}_{0}$, but does depend on those moduli appearing in $\tilde{b}_{2}$ and $\tilde{b}_{3}$. Turning off $\tilde{b}_{3}$ and $\tilde{b}_{2}$ in succession would enhance the generic curve of $E_{6}$ singularities where the GUT stack intersects the instanton to $E_{7}$ and then $E_{8}$. The moduli appearing in $\tilde{b}_{0}$ are those which ensure that the singularity type of the curve does not enhance "past $E_{8}$ " to a non-Kodaira singularity. Thus, only the moduli $\chi_{i}$ and $\phi_{i}$ which control the Higgsing of the $E_{8}$ curve appear in the Pfaffian.

Though we have a qualitative understanding of the moduli which appear in the Pfaffian, there is actually a simple geometric structure which determines the entirety of the polynomial $f_{E 8}$. It is none other than the determinant of 62 .

$$
M \equiv\left(\begin{array}{ccccc}
\phi_{4} & \phi_{3} & \phi_{2} & \phi_{1} & 0 \\
0 & \phi_{4} & \phi_{3} & \phi_{2} & \phi_{1} \\
\chi_{3} & \chi_{2} & \chi_{1} & 0 & 0 \\
0 & \chi_{3} & \chi_{2} & \chi_{1} & 0 \\
0 & 0 & \chi_{3} & \chi_{2} & \chi_{1}
\end{array}\right)
$$


so that $f_{E 8}=\operatorname{det}(M)$ and therefore the prefactor $A \sim \operatorname{det}(M)^{4}$. Matrices of this form are well-known in elimination theory as Sylvester matrices, and $M$ itself is the Sylvester matrix of the two polynomials $\tilde{b}_{2}$ and $\tilde{b}_{3}$. The determinant of the Sylvester matrix of two polynomials is the resultant of those two polynomials, which has a zero if and only if the two polynomials have a common zero. We therefore have a precise algebraic understanding of $f_{E 8}$. It is the resultant of $\tilde{b}_{2}$ and $\tilde{b}_{3}$, and thus the prefactor vanishes if and only if $\tilde{b}_{2}$ and $\tilde{b}_{3}$ have a common zero.

The algebraic statement $f_{E 8}=\operatorname{Res}\left(\tilde{b}_{2}, \tilde{b}_{3}\right)$ has a concrete geometric realization in F-theory. Suppose that we are at a point in the moduli space which gives a zero of $f_{E 8}$, so that there is a point $\left(x_{2}^{*}, x_{3}^{*}\right)$ in $\Sigma$ along which $\tilde{b}_{2}$ and $\tilde{b}_{3}$ have a common zero. A simultaneous zero of $\tilde{b}_{2}$ and $\tilde{b}_{3}$ enhance the singularity to $E_{8}$, which in this case occurs at a point. Thus, in this example an F-theoretic understanding of the prefactor is

$$
A \sim f_{E 8}^{4}=0 \quad \Leftrightarrow \quad M 5 \supset \text { pt of } E_{8} \text { enhancement. }
$$

In addition to being beautiful, connections between superpotential zeroes and points of $E_{8}$ could also have phenomenological significance, as points of $E_{8}$ are known to give rise to interesting physics in $S U(5)$ GUT models 61. While we see a direct correspondence in this example, the phenomenon is more generic, and we refer the reader to section 7 for a discussion. There we identify one structure governing the relationship between superpotential zeroes and points of $E_{8}$. We will show that the connection can also exist for other gauge groups, including $G_{4 d}=S U(5)$.

\subsection{Example Three: A New Prefactor}

Let us briefly consider one final example of a prefactor computation in F-theory. Consider an F-theory compactification on the same base $B_{3}$ as in section 8.2. Again let the instanton wrap $D 3 \equiv\left\{x_{1}=0\right\}$, but this time consider an $E_{6}$ GUT along $B_{2} \equiv\left\{x_{0}=0\right\} 25$. Having changed the location of the GUT stack, in this example we have

$$
\eta=19 X+13 E_{1}+13 E_{2} \quad c_{1, B_{3}}=\left.\left(3 x+2 E_{1}+2 E_{2}\right)\right|_{B_{2}},
$$

and for $\lambda=\frac{3}{2}$ a simple computation as in the other two examples gives that $\{n, \lambda, r, \chi\}=$ $\left\{3, \frac{3}{2}, 6,1\right\}$. We then have that

$$
\left[c_{l o c}\right]=3 s+6 F \quad \mathcal{L}_{A}=\left.\mathcal{O}_{\mathcal{E}}(6 s-2 F)\right|_{c_{l o c}},
$$

which is enough data to set up a mathematical computation of line bundle cohomology using the techniques of [69]. Unlike the previous two examples, however, this example has not been computed in the literature, and we would like to give the result. Note that this geometry exemplifies the Pfaffian whose data is given in row 3 of 1 .

Per usual in $E_{6}$ models, the defining equation of $c_{l o c}$ is

$$
f_{c_{l o c}}=\tilde{b}_{0} v^{3}+\tilde{b}_{2} v x+\tilde{b}_{3} y
$$

${ }^{25}$ Throughout we have used $z=0$ as the GUT locus in $B_{3}$, but in this example we use $x_{1}=0$. The corresponding equations for the geometries used throughout must replace $z$ by $x_{1}$ for this example only. 
where $b_{m} \in H^{0}\left(B_{3}, \mathcal{O}_{B_{3}}\left((19-3 m) X+(13-2 m) E_{1}+(13-2 m) E_{2}\right)\right)$ in this example. Restricting to the intersection of the GUT stack and the instanton at $\left\{x_{0}=x_{1}=0\right\}$ we can write down the form of the monomials in $\tilde{b}_{q}$

$$
\begin{array}{ll}
\tilde{b}_{0} \text { monomials } \sim x_{2}^{13-i} x_{3}^{i} z^{13} x_{5}^{6-i} & i=0 \ldots 6 \\
\tilde{b}_{2} \text { monomials } \sim x_{2}^{9-i} x_{3}^{i} z^{9} x_{5}^{4-i} & i=0 \ldots 4 \\
\tilde{b}_{3} \text { monomials } \sim x_{2}^{7-i} x_{3}^{i} z^{7} x_{5}^{3-i} & i=0 \ldots 3
\end{array}
$$

where the range of $i$ has been chosen to ensure that the monomials are global sections. From the Stanley-Reisner ideal it can be seen that when $x_{0}=x_{1}=0$ one can use the GLSM relations to set $x_{2}=z=1$ leaving two free coordinates $x_{3}$ and $x_{5}$ which are the coordinates on $\Sigma=\mathbb{P}^{1}$. We can then write down

$$
\begin{aligned}
& \tilde{b}_{0}=\alpha_{1} x_{3}^{6}+\alpha_{2} x_{3}^{5} x_{5}+\alpha_{3} x_{3}^{4} x_{5}^{2}+\alpha_{4} x_{3}^{3} x_{5}^{3}+\alpha_{5} x_{3}^{2} x_{5}^{4}+\alpha_{6} x_{3}^{1} x_{5}^{5}+\alpha_{7} x_{5}^{6} \\
& \tilde{b}_{2}=\beta_{1} x_{3}^{4}+\beta_{2} x_{3}^{3} x_{5}+\beta_{3} x_{3}^{2} x_{5}^{2}+\beta_{4} x_{3} x_{5}^{3}+\beta_{5} x_{5}^{4} \\
& \tilde{b}_{3}=\gamma_{1} x_{3}^{3}+\gamma_{2} x_{3}^{2} x_{5}+\gamma_{3} x_{3} x_{5}^{2}+\gamma_{4} x_{5}^{3} .
\end{aligned}
$$

and we thus know $f_{c_{l o c}}$ explicitly.

Let us now perform the computation of the moduli-dependent prefactor. We are tasked with computing the cohomology $H^{i}\left(c_{l o c}, \mathcal{L}_{A}\right)$, and we will utilize the Koszul sequence

$$
0 \rightarrow \mathcal{O}_{\mathcal{E}}(3 s-8 F) \stackrel{f_{c_{l o c}}}{\longrightarrow} \mathcal{O}_{\mathcal{E}}(6 s-2 F) \rightarrow \mathcal{L}_{A} \rightarrow 0 .
$$

Via a Leray spectral sequence 26 it can be shown that

$H^{0}\left(\mathcal{E}, \mathcal{O}_{\mathcal{E}}(3 s-8 F)\right)=H^{2}\left(\mathcal{E}, \mathcal{O}_{\mathcal{E}}(3 s-8 F)\right)=H^{0}\left(\mathcal{E}, \mathcal{O}_{\mathcal{E}}(6 s-2 F)\right)=H^{2}\left(\mathcal{E}, \mathcal{O}_{\mathcal{E}}(6 s-2 F)\right)=0$

and therefore the long exact sequence in cohomology corresponding to 8.39 simplifies to

$$
0 \rightarrow H^{0}\left(c_{l o c}, \mathcal{L}_{A}\right) \rightarrow H^{1}\left(\mathcal{E}, \mathcal{O}_{\mathcal{E}}(3 s-8 F)\right) \stackrel{f}{\rightarrow} H^{1}\left(\mathcal{E}, \mathcal{O}_{\mathcal{E}}(6 s-2 F)\right) \rightarrow H^{1}\left(c_{l o c}, \mathcal{L}_{A}\right) \rightarrow 0
$$

where $f$ is the moduli-dependent matrix map induced by $f_{c_{l o c}}$ whose determinant is the prefactor. Via a Leray spectral sequence, it can be shown that $H^{1}\left(\mathcal{E}, \mathcal{O}_{\mathcal{E}}(3 s-8 F)\right)=$ $H^{1}\left(\Sigma, \pi_{3, F *} \mathcal{O}_{\mathcal{E}}(3 s-8 F)\right)$ and $H^{1}\left(\mathcal{E}, \mathcal{O}_{\mathcal{E}}(6 s-2 F)\right)=H^{1}\left(\Sigma, \pi_{3, F *} \mathcal{O}_{\mathcal{E}}(6 s-2 F)\right)$ where

$$
\begin{aligned}
& \pi_{3, F *} \mathcal{O}_{\mathcal{E}}(3 s-8 F)=\mathcal{O}_{\Sigma}(-8) \oplus \mathcal{O}_{\Sigma}(-10) \oplus \mathcal{O}_{\Sigma}(-11) \\
& \pi_{3, F *} \mathcal{O}_{\mathcal{E}}(6 s-2 F)=\mathcal{O}_{\Sigma}(-2) \oplus \mathcal{O}_{\Sigma}(-4) \oplus \mathcal{O}_{\Sigma}(-5) \mathcal{O}_{\Sigma}(-6) \oplus \mathcal{O}_{\Sigma}(-7) \oplus \mathcal{O}_{\Sigma}(-8)
\end{aligned}
$$

and we therefore see $h^{1}\left(\mathcal{E}, \mathcal{O}_{\mathcal{E}}(3 s-8 F)\right)=h^{1}\left(\mathcal{E}, \mathcal{O}_{\mathcal{E}}(6 s-2 F)\right)=26$ using (8.42) and the fact that $h^{1}\left(\mathbb{P}^{1}, \mathcal{O}(-i)\right)=i-1$. This means the $f$ is a $26 \times 26$ matrix.

We have worked out a few more details of the computation than in previous examples, since this is a new example. We refer the reader to [59] for more details. Using the same

\footnotetext{
${ }^{26}$ For a collection of useful results regarding line bundle cohomology on $\mathcal{E}$ and Leray spectral sequences see appendix $\mathrm{C}$ of 62 .
} 
techniques as in that work, the moduli-dependent matrix $f$ can be constructed explicitly, and (in a particular basis) it is given by

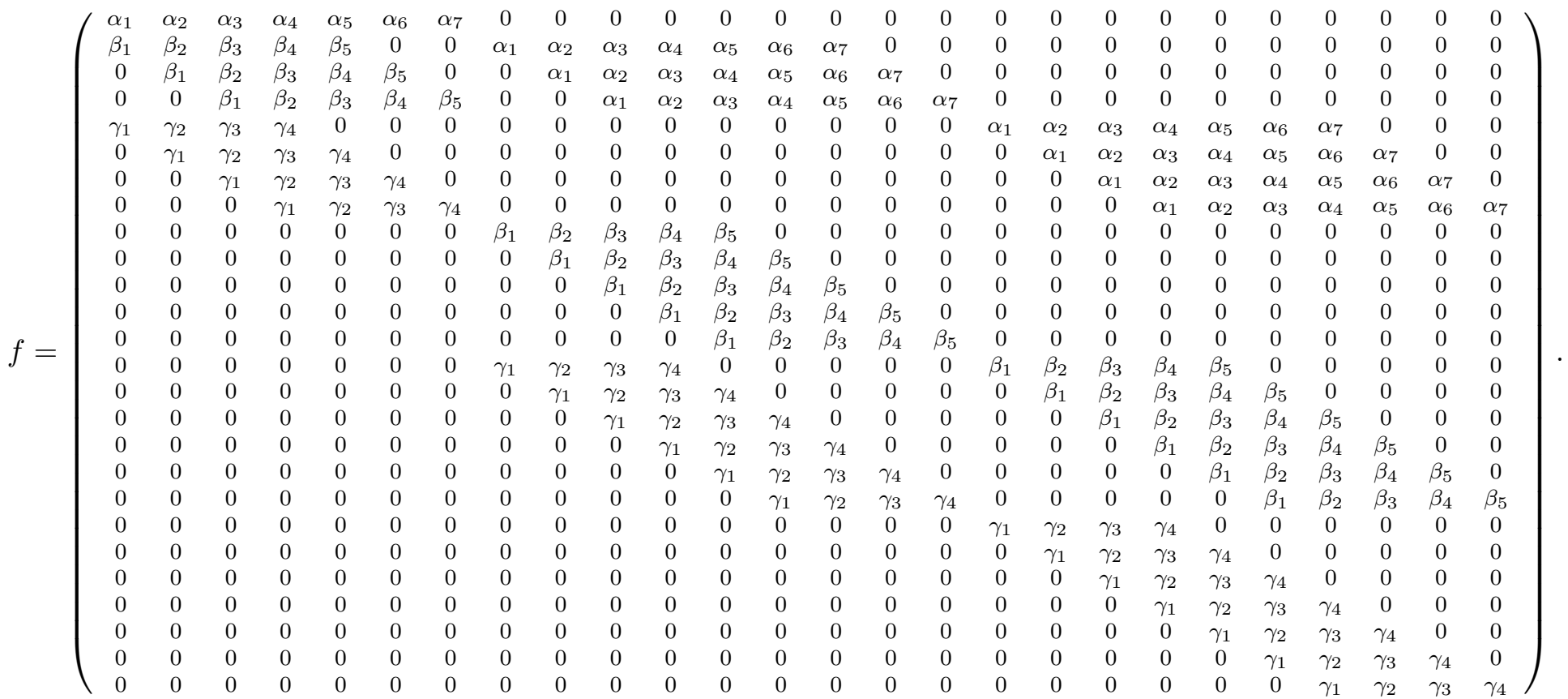

Given this expression, the matrix can be plugged into one's favorite computer algebra system and a few hours later the prefactor is found to be

$$
A=\operatorname{det}(f)=0 .
$$

Thus, there exists a zero mode for all $\alpha_{i}, \beta_{i}$ and $\gamma_{i}$ moduli which one would not have known about without constructing the maps explicitly. This zero mode causes the prefactor to vanish for all of these moduli. It is worth noting that this prefactor occurred very frequently in the scan we performed in section 6 and clearly has difficult implications for moduli stabilization with the associated instanton. Though this occurred while studying a seven-brane dependent instanton prefactor in F-theory, this mathematical phenomenon should occur in other compactifications as well and one should not simply assume an $\mathcal{O}(1)$ prefactor.

\section{Acknowledgments}

We thank Lara Anderson, Andres Collinucci, Iñaki García-Etxebarria, James Gray, Thomas Grimm, Jonathan Heckman, Max Kerstan, Liam McAllister, Paul McGuirk, Natalia Saulina, Sakura Schäfer-Nameki, Sav Sethi, Gary Shiu, Angel Uranga, Timo Weigand, and Cumrun Vafa for conversations regarding this work. J.H. is particularly grateful to Denis Klevers for many long and useful conversations and Dave Morrison for a discussion of the draft. R.D. thanks his long time collaborator Martijn Wijnholt. J.H. and M.C. are supported by DOE grant DOE-EY-76-02-3071. J.H. also acknowledges support from NSF grant PHY11-25915 and a DOE graduate fellowship. J.M. is supported by DOE grant DE-FG02-90ER-40560 and NSF grant PHY-0855039. R.D. acknowledges partial support from NSF grants DMS-0908487 and RTG-0636606. M.C. is also supported by the Fay R. and Eugene L. Langberg Endowed Chair and the Slovenian Research Agency (ARRS). 


\section{A Calabi-Yau Resolutions and the Spectral Di- visor}

In this Appendix, we describe a crepant resolution of the 4-fold $Y_{4}(3.2)$ and the $d P_{9}$ fibration $Y_{4}^{\prime}(4.3)$. Further, we provide a detailed description of the spectral divisor $\mathcal{C}_{F}$ (3.4), its relation to the cylinder (4.6), as well as the emergence of the compact Higgs bundle spectral cover (3.17) and its relation to the heterotic spectral cover (2.3).

\section{A.1 Resolving 4-folds with a surface of $E_{6}$ singularities}

We now proceed to consider a 4 -fold $\mathcal{Y}_{4}$ that is elliptically fibered with section over a base $B_{3}$ and exhibits a surface of $E_{6}$ singularities. We realize $\mathcal{Y}_{4}$ as a hypersurface in a $\mathbb{P}_{1,2,3}^{2}$ bundle $\mathcal{W}_{5}$ over $B_{3}$ with weighted homogeneous coordinates $[v, x, y]$ transforming as sections of the indicated bundles

\begin{tabular}{c|c} 
Section & Bundle \\
\hline$v$ & $\mathcal{O}(\sigma)$ \\
$x$ & $\mathcal{O}(2 \sigma+2 \mathcal{D})$ \\
$y$ & $\mathcal{O}(3 \sigma+3 \mathcal{D})$
\end{tabular}

where $\mathcal{D}$ is some divisor class in $B_{3}$. Keeping $\mathcal{D}$ general will allow us to describe $\mathcal{Y}_{4}$ "s that are Calabi-Yau as well as $\mathcal{Y}_{4}$ 's that are not Calabi-Yau, such as the $d P_{9}$ fibration $Y_{4}^{\prime}$ (4.3) that emerges in the stable degeneration limit of $Y_{4}$ (3.2). We take our 4-fold $\mathcal{Y}_{4}$ to be the hypersurface defined by

$$
y^{2}=x^{3}+f x z^{4} v^{4}+g z^{6} v^{6}+z^{2} v^{3}\left[b_{0} z^{3} v^{3}+b_{2} z v x+b_{3} y\right]
$$

where the new objects are sections of the indicated bundles

\begin{tabular}{c|c} 
Section & Bundle \\
\hline$z$ & $\mathcal{O}\left(B_{2}\right)$ \\
$b_{m}$ & $\mathcal{O}\left([6-m] \mathcal{D}-[5-m] B_{2}\right)$ \\
$f$ & $\mathcal{O}\left(4\left(\mathcal{D}-B_{2}\right)\right)$ \\
$g$ & $\mathcal{O}\left(6\left(\mathcal{D}-B_{2}\right)\right)$
\end{tabular}

for $B_{2}$ a surface in $B_{3}$ along which $\mathcal{Y}_{4}$ exhibits an $E_{6}$ singularity. The hypersurface $\mathcal{Y}_{4}$ is in the class $6(\sigma+\mathcal{D})$ in $\mathcal{W}_{5}$ and has first Chern class

$$
c_{1}\left(\mathcal{Y}_{4}\right)=c_{1}\left(B_{3}\right)-\mathcal{D}
$$

As usual, a crepant resolution of the singularities along $B_{2}$ can be obtained by performing a series of blow-ups in $\mathcal{W}_{5}$ and passing from $\mathcal{Y}_{4}$ to its proper transform $\tilde{\mathcal{Y}}_{4}$. We now describe this in detail. While this work was in progress, the resolution of elliptically fibered Calabi-Yau's with a surface of $E_{6}$ singularities was also studied in [36].

\section{Blow-up 1}

The first step is to blow up $\mathcal{W}_{5}$ along the surface $x=y=z=0$ to get the once blown-up space $\mathcal{W}_{5}^{(1)}$. This gives an exceptional divisor $E_{1}$. The holomorphic sections $x, y$, and $z$ 
factor in $\mathcal{W}_{5}^{(1)}$ as

$$
\begin{aligned}
& x=x_{1} \delta_{1} \\
& y=y_{1} \delta_{1} \\
& z=z_{1} \delta_{1}
\end{aligned}
$$

for $\delta_{1}$ the unique holomorphic section of $\mathcal{O}\left(E_{1}\right)$ whose vanishing defines $E_{1}$. The new objects in (A.5) are holomorphic sections of the indicated bundles

\begin{tabular}{c|c} 
Section & Bundle \\
\hline$x_{1}$ & $\mathcal{O}\left(2[\sigma+\mathcal{D}]-E_{1}\right)$ \\
$y_{1}$ & $\mathcal{O}\left(3[\sigma+\mathcal{D}]-E_{1}\right)$ \\
$z_{1}$ & $\mathcal{O}\left(B_{2}-E_{1}\right)$ \\
$\delta_{1}$ & $\mathcal{O}\left(E_{1}\right)$
\end{tabular}

It will be helpful in the following to say something about the geometry of $E_{1}$. Before the blow-up, $x=y=z=0$ is a copy of $B_{2}$ is $E_{1}$ is the projectivization of the normal bundle of this copy of $B_{2}$ inside $\mathcal{W}_{5}$. In particular, we have

$$
\begin{aligned}
E_{1} & =\mathbb{P}\left(\left.\left.\left.\mathcal{O}(2[\sigma+\mathcal{D}])\right|_{B_{2}} \oplus \mathcal{O}(3[\sigma+\mathcal{D}])\right|_{B_{2}} \oplus \mathcal{O}\left(B_{2}\right)\right|_{B_{2}}\right) \\
& =\mathbb{P}\left(\left.\left.\left.\mathcal{O}(2 \mathcal{D})\right|_{B_{2}} \oplus \mathcal{O}(3 \mathcal{D})\right|_{B_{2}} \oplus \mathcal{O}\left(B_{2}\right)\right|_{B_{2}}\right)
\end{aligned}
$$

where we have used the fact that $\left.\sigma\right|_{x=y=0}=0$. The restrictions of $x_{1}, y_{1}$, and $z_{1}$ give homogeneous coordinates on the $\mathbb{P}^{2}$ fiber of $E_{1}$. From the description of $E_{1}$ in (A.7), we can specify the bundles on $E_{1}$ of which $x_{1}, y_{1}$, and $z_{1}$ are sections by

\begin{tabular}{c|c} 
Section & Bundle on $E_{1}$ \\
\hline$x_{1}$ & $\mathcal{O}_{E_{1}}\left(\sigma_{1}+2 \mathcal{D}\right)$ \\
$y_{1}$ & $\mathcal{O}_{E_{1}}\left(\sigma_{1}+3 \mathcal{D}\right)$ \\
$z_{1}$ & $\mathcal{O}_{E_{1}}\left(\sigma_{1}+B_{2}\right)$
\end{tabular}

where $\sigma_{1}$ is the 'new' divisor class on $E_{1}$ that is not pulled back from $B_{2}$. Using the implied restrictions

$$
\begin{aligned}
{\left.\left[2(\sigma+\mathcal{D})-E_{1}\right]\right|_{E_{1}} } & =\sigma_{1}+2 \mathcal{D} \\
{\left.\left[3(\sigma+\mathcal{D})-E_{1}\right]\right|_{E_{1}} } & =\sigma_{1}+3 \mathcal{D} \\
{\left.\left[B_{2}-E_{1}\right]\right|_{E_{1}} } & =\sigma_{1}+B_{2}
\end{aligned}
$$

and the fact that $\left.\sigma\right|_{E_{1}}=0$, we see that $\sigma_{1}$ is nothing more than

$$
\sigma_{1}=-\left.E_{1}\right|_{E_{1}}
$$

As such, the normal bundle of $E_{1}$ in $\mathcal{W}_{5}^{(1)}$ is given by

$$
N_{E_{1} / \mathcal{W}_{5}^{(1)}}=\mathcal{O}_{E_{1}}\left(-\sigma_{1}\right)
$$




\section{Blow-up 2}

The second step in our procedure is to blow-up along the 3 -fold $y_{1}=\delta_{1}=0$ in $\mathcal{W}_{5}^{(1)}$ to get the twice blown-up space $\mathcal{W}_{5}^{(2)}$. This yields an exceptional divisor $E_{2}$. The holomorphic sections $y_{1}$ and $\delta_{1}$ factor in $\mathcal{W}_{5}^{(2)}$ as

$$
\begin{aligned}
& y_{1}=y_{12} \delta_{2} \\
& \delta_{1}=\delta_{12} \delta_{2}
\end{aligned}
$$

where the new objects are sections of the indicated bundles

\begin{tabular}{c|c} 
Section & Bundle \\
\hline$y_{12}$ & $\mathcal{O}\left(3[\sigma+\mathcal{D}]-E_{1}-E_{2}\right)$ \\
$\delta_{12}$ & $\mathcal{O}\left(E_{1}-E_{2}\right)$ \\
$\delta_{2}$ & $\mathcal{O}\left(E_{2}\right)$
\end{tabular}

The exceptional divisor $E_{2}$ is the projectivization of the normal bundle of the 3 -fold $y_{1}=\delta_{1}=0$ inside $\mathcal{W}_{5}^{(1)}$. This 3 -fold, which we call $X_{3}^{(2)}$, is the divisor defined by $y_{1}=0$ inside $E_{1}$ and is in the class $\sigma_{1}+3 \mathcal{D}$. From (A.8), we see that it is the following $\mathbb{P}^{1}$-bundle over $B_{2}$

$$
X_{3}^{(2)}=\mathbb{P}\left(\mathcal{O}_{B_{2}}(2 \mathcal{D}) \oplus \mathcal{O}_{B_{2}}\left(B_{2}\right)\right)
$$

with $x_{1}$ and $z_{1}$ providing homogeneous coordinates on the $\mathbb{P}^{1}$ fiber. The normal bundle of $X_{3}^{(2)}$ inside $\mathcal{W}_{5}^{(1)}$ is simply

$$
\begin{aligned}
N_{X_{3}^{(2)} / \mathcal{W}_{5}^{(1)}} & =\left.N_{X_{3}^{(2)} / E_{1}} \oplus N_{E_{1} / \mathcal{W}_{5}^{(1)}}\right|_{X_{3}^{(2)}} \\
& =\left.\left.\mathcal{O}_{E_{1}}\left(\sigma_{1}+3 \mathcal{D}\right)\right|_{X_{3}^{(2)}} \oplus \mathcal{O}_{E_{1}}\left(-\sigma_{1}\right)\right|_{X_{3}^{(2)}}
\end{aligned}
$$

and $E_{2}$ is the projectivization of this

$$
E_{2}=\mathbb{P}\left(\left.\left.\mathcal{O}_{E_{1}}\left(\sigma_{1}+3 \mathcal{D}\right)\right|_{X_{3}^{(2)}} \oplus \mathcal{O}_{E_{1}}\left(-\sigma_{1}\right)\right|_{X_{3}^{(2)}}\right)
$$

The sections $y_{12}, \delta_{12}$ restrict to homogeneous coordinates on the $\mathbb{P}^{1}$ fiber. In total $x_{1}, z_{1}, y_{12}, \delta_{12}$ restrict to sections of the following bundles on $E_{2}$

\begin{tabular}{c|c} 
Section & Bundle on $E_{2}$ \\
\hline$x_{1}$ & $\mathcal{O}_{E_{2}}\left(\sigma_{1}+2 \mathcal{D}\right)$ \\
$z_{1}$ & $\mathcal{O}_{E_{2}}\left(\sigma_{1}+B_{2}\right)$ \\
$y_{12}$ & $\mathcal{O}_{E_{2}}\left(\sigma_{2}+\sigma_{1}+3 \mathcal{D}\right)$ \\
$\delta_{12}$ & $\sigma_{2}-\sigma_{1}$
\end{tabular}

where $\sigma_{2}$ is the 'new' divisor class associated with the blown-up $\mathbb{P}^{1}$ fiber. In particular,

$$
\sigma_{1}=-\left.E_{1}\right|_{E_{2}} \quad \sigma_{2}=-\left.E_{2}\right|_{E_{2}}
$$

Since $X_{3}^{(2)}$ is itself a $\mathbb{P}^{1}$-fibration over $B_{2}, E_{2}$ is a $\mathbb{P}^{1}$-fibration over a $\mathbb{P}^{1}$-fibration over $B_{2}$ or, equivalently, an $\mathbb{F}_{n}$-fibration over $B_{2}$. Inside the $\mathbb{F}_{n}$, the divisor $\sigma_{1}$ gives the $\mathbb{P}^{1}$ fiber and the divisor $\sigma_{2}$ gives the $\mathbb{P}^{1}$ base. in fact, we can verify that $E_{2}$ is an $\mathbb{F}_{2}$ fibration since $\sigma_{2}^{2}=-2$ in the fiber. The normal bundle of $E_{2}$ inside $\mathcal{W}_{5}^{(2)}$ is simply

$$
N_{E_{2} / \mathcal{W}_{5}^{(2)}}=\left.\mathcal{O}\left(E_{2}\right)\right|_{E_{2}}=\mathcal{O}_{E_{2}}\left(-\sigma_{2}\right)
$$




\section{Blow-up 3}

The third step is to blow-up $\mathcal{W}_{5}^{(2)}$ along the 3 -fold $x_{1}=\delta_{2}=0$ to get the 3 -times blownup space $\mathcal{W}_{5}^{(3)}$. This gives an exceptional divisor $E_{3}$. The sections $x_{1}$ and $\delta_{2}$ factor in $\mathcal{W}_{5}^{(3)}$ as

$$
\begin{aligned}
x_{1} & =x_{13} \delta_{3} \\
\delta_{2} & =\delta_{23} \delta_{3}
\end{aligned}
$$

where the new objects are sections of the indicated bundles

\begin{tabular}{c|c} 
Section & Bundle \\
\hline$x_{13}$ & $\mathcal{O}\left(2[\sigma+\mathcal{D}]-E_{1}-E_{3}\right)$ \\
$\delta_{23}$ & $\mathcal{O}\left(E_{2}-E_{3}\right)$ \\
$\delta_{3}$ & $\mathcal{O}\left(E_{3}\right)$
\end{tabular}

The exceptional divisor $E_{3}$ is the projectivization of the normal bundle of the 3 -fold $x_{1}=\delta_{2}=0$ inside $\mathcal{W}_{5}^{(2)}$. This 3 -fold, which we call $X_{3}^{(3)}$, is the divisor defined by $x_{1}$ inside $E_{2}$ and is in the class $\sigma_{1}+2 \mathcal{D}$. We recall that the holomorphic section $z_{1}$ in (A.17) cannot vanish along $x_{1}=0$ in $E_{2}$ so $\left(\sigma_{1}+2 \mathcal{D}\right) \cdot E_{2}\left(\sigma_{1}+B_{2}\right)=0$. This means that $\left.\sigma_{1}\right|_{X_{3}^{(3)}}=-\left.B_{2}\right|_{X_{3}^{(3)}}$ and, consequently, that $X_{3}^{(3)}$ is the following $\mathbb{P}^{1}$-bundle over $B_{2}$

$$
X_{3}^{(3)}=\mathbb{P}\left(\mathcal{O}_{B_{2}}\left(3 \mathcal{D}-B_{2}\right) \oplus \mathcal{O}_{B_{2}}\left(B_{2}\right)\right)
$$

with $y_{12}$ and $\delta_{12}$ providing homogeneous coordinates on the $\mathbb{P}^{1}$ fiber. The normal bundle of $X_{3}^{(3)}$ in $\mathcal{W}_{5}^{(2)}$ is simply

$$
\begin{aligned}
N_{X_{3}^{(3)} / \mathcal{W}_{5}^{(2)}} & =\left.N_{X_{3}^{(3)} / E_{2}} \oplus N_{E_{2} / \mathcal{W}_{5}^{(2)}}\right|_{X_{3}^{(3)}} \\
& =\left.\left.\mathcal{O}_{E_{2}}\left(\sigma_{1}+2 \mathcal{D}\right)\right|_{X_{3}^{(3)}} \oplus \mathcal{O}_{E_{2}}\left(-\sigma_{2}\right)\right|_{X_{3}^{(3)}} \\
& =\left.\left.\mathcal{O}_{E_{2}}\left(2 \mathcal{D}-B_{2}\right)\right|_{X_{3}^{(3)}} \oplus \mathcal{O}_{E_{2}}\left(-\sigma_{2}\right)\right|_{X_{3}^{(3)}}
\end{aligned}
$$

and $E_{3}$ is the projectivization of this

$$
E_{3}=\mathbb{P}\left(\left.\left.\mathcal{O}_{E_{2}}\left(2 \mathcal{D}-B_{2}\right)\right|_{X_{3}^{(3)}} \oplus \mathcal{O}_{E_{2}}\left(-\sigma_{2}\right)\right|_{X_{3}^{(3)}}\right)
$$

The sections $x_{13}$ and $\delta_{23}$ restrict to homogeneous coordinates on the $\mathbb{P}^{1}$ fiber. In total, $y_{12}, \delta_{12}, x_{13}, \delta_{23}$ restrict to sections of the following bundles on $E_{3}$

\begin{tabular}{c|c} 
Section & Bundle on $E_{3}$ \\
\hline$y_{12}$ & $\mathcal{O}_{E_{3}}\left(\sigma_{2}+3 \mathcal{D}-B_{2}\right)$ \\
$\delta_{12}$ & $\mathcal{O}_{E_{3}}\left(\sigma_{2}+B_{2}\right)$ \\
$x_{13}$ & $\mathcal{O}_{E_{3}}\left(\sigma_{3}+2 \mathcal{D}-B_{2}\right)$ \\
$\delta_{23}$ & $\mathcal{O}_{E_{3}}\left(\sigma_{3}-\sigma_{2}\right)$
\end{tabular}

where $\sigma_{3}$ is the 'new' divisor class associated with the blown-up $\mathbb{P}^{1}$ fiber. In particular,

$$
\sigma_{2}=-\left.E_{2}\right|_{E_{3}} \quad \sigma_{3}=-\left.E_{3}\right|_{E_{3}}
$$


Since $X_{3}^{(3)}$ is itself a $\mathbb{P}^{1}$-fibration over $B_{2}, E_{3}$ is an $\mathbb{F}_{n}$-fibration over $B_{2}$. Inside the $\mathbb{F}_{n}$, the divisor $\sigma_{2}$ gives the $\mathbb{P}^{1}$ fiber class and $\sigma_{3}$ the $\mathbb{P}^{1}$ base. The normal bundle of $E_{3}$ inside $\mathcal{W}_{5}^{(3)}$ is simply

$$
N_{E_{3} / \mathcal{W}_{5}^{(3)}}=\left.\mathcal{O}\left(E_{3}\right)\right|_{E_{3}}=\mathcal{O}_{E_{3}}\left(-\sigma_{3}\right)
$$

\section{Blow-up 4}

The fourth step is to blow-up $\mathcal{W}_{5}^{(3)}$ along the 3 -fold $y_{12}=\delta_{3}=0$ to get the 4 -times blown-up space $\mathcal{W}_{5}^{(4)}$. This gives an exceptional divisor $E_{4}$. The sections $y_{12}$ and $\delta_{3}$ factor in $\mathcal{W}_{5}^{(4)}$ as

$$
\begin{aligned}
y_{12} & =y_{124} \delta_{4} \\
\delta_{3} & =\delta_{34} \delta_{4}
\end{aligned}
$$

where the new objects are sections of the indicated bundles

\begin{tabular}{c|c} 
Section & Bundle \\
\hline$y_{124}$ & $\mathcal{O}\left(3[\sigma+\mathcal{D}]-E_{1}-E_{2}-E_{4}\right)$ \\
$\delta_{34}$ & $\mathcal{O}\left(E_{3}-E_{4}\right)$ \\
$\delta_{4}$ & $\mathcal{O}\left(E_{4}\right)$
\end{tabular}

The exceptional divisor $E_{4}$ is the projectivization of the normal bundle of the 3-fold $y_{12}=\delta_{4}=0$ inside $\mathcal{W}_{5}^{(3)}$. This 3 -fold, which we call $X_{3}^{(4)}$, is the divisor defined by $y_{12}=0$ inside $E_{3}$ and is in the class $\sigma_{2}+3 \mathcal{D}-B_{2}$. We recall that the holomorphic section $\delta_{12}$ in (A.25) cannot vanish along $y_{12}=0$ in $E_{3}$ so $\left(\sigma_{2}+3 \mathcal{D}-B_{2}\right) \cdot E_{3}\left(\sigma_{2}+B_{2}\right)=0$. This means that $\left.\sigma_{2}\right|_{X_{3}^{(4)}}=-\left.B_{2}\right|_{X_{3}^{(4)}}$ and, consequently, that $X_{3}^{(4)}$ is the following $\mathbb{P}^{1}$ bundle over $B_{2}$

$$
X_{3}^{(4)}=\mathbb{P}\left(\mathcal{O}_{B_{2}}\left(2 \mathcal{D}-B_{2}\right) \oplus \mathcal{O}_{B_{2}}\left(B_{2}\right)\right)
$$

with $x_{13}$ and $\delta_{23}$ providing homogeneous coordinates on the $\mathbb{P}^{1}$ fiber. The normal bundle of $X_{3}^{(4)}$ in $\mathcal{W}_{5}^{(3)}$ is simply

$$
\begin{aligned}
N_{X_{3}^{(4)} / \mathcal{W}_{5}^{(3)}} & =\left.N_{X_{3}^{(4)} / E_{3}} \oplus N_{E_{3} / \mathcal{W}_{5}^{(3)}}\right|_{X_{3}^{(4)}} \\
& =\left.\left.\mathcal{O}\left(E_{3}\right)\left(\sigma_{2}+3 \mathcal{D}-B_{2}\right)\right|_{X_{3}^{(4)}} \oplus \mathcal{O}_{E_{3}}\left(-\sigma_{3}\right)\right|_{X_{3}^{(4)}} \\
& =\left.\left.\mathcal{O}\left(E_{3}\right)\left(3 \mathcal{D}-2 B_{2}\right)\right|_{X_{3}^{(4)}} \oplus \mathcal{O}_{E_{3}}\left(-\sigma_{3}\right)\right|_{X_{3}^{(4)}}
\end{aligned}
$$

and $E_{4}$ is the projectivization of this

$$
E_{4}=\mathbb{P}\left(\left.\left.\mathcal{O}\left(E_{3}\right)\left(3 \mathcal{D}-2 B_{2}\right)\right|_{X_{3}^{(4)}} \oplus \mathcal{O}_{E_{3}}\left(-\sigma_{3}\right)\right|_{X_{3}^{(4)}}\right)
$$

The sections $y_{124}$ and $\delta_{34}$ restrict to homogeneous coordinates on the $\mathbb{P}^{1}$ fiber. In total, $x_{13}, \delta_{23}, y_{124}, \delta_{34}$ restrict to sections of the following bundles on $E_{4}$

\begin{tabular}{c|c} 
Section & Bundle on $E_{4}$ \\
\hline$x_{13}$ & $\mathcal{O}_{E_{4}}\left(\sigma_{3}+2 \mathcal{D}-B_{2}\right)$ \\
$\delta_{23}$ & $\mathcal{O}_{E_{4}}\left(\sigma_{3}+B_{2}\right)$ \\
$y_{124}$ & $\mathcal{O}_{E_{4}}\left(\sigma_{4}+3 \mathcal{D}-2 S_{2}\right)$ \\
$\delta_{34}$ & $\mathcal{O}_{E_{4}}\left(\sigma_{4}-\sigma_{3}\right)$
\end{tabular}


where $\sigma_{4}$ is the 'new' divisor class associated with the blown-up $\mathbb{P}^{1}$ fiber. In particular,

$$
\sigma_{3}=-\left.E_{3}\right|_{E_{4}} \quad \sigma_{4}=-\left.E_{4}\right|_{E_{4}}
$$

Since $X_{3}^{(4)}$ is itself a $\mathbb{P}^{1}$-fibration over $B_{2}, E_{4}$ is an $\mathbb{F}_{n}$-fibration over $B_{2}$. Inside the $\mathbb{F}_{n}$, the divisor $\sigma_{3}$ gives the $\mathbb{P}^{1}$ fiber class and $\sigma_{4}$ the $\mathbb{P}^{1}$ base. The normal bundle of $E_{4}$ inside $\mathcal{W}_{5}^{(4)}$ is simply

$$
N_{E_{4} / \mathcal{W}_{5}^{(4)}}=\left.\mathcal{O}\left(E_{4}\right)\right|_{E_{4}}=\mathcal{O}_{E_{4}}\left(-\sigma_{4}\right)
$$

\section{The last two blow-ups}

The four blow-ups that we have described so far are sufficient to resolve a 4-fold with a surface of $S U(5)$ singularities along $B_{2}$ [33. Further, our detailed description of the exceptional divisors $E_{1}, E_{2}, E_{3}$, and $E_{4}$ will be sufficient to give a careful description of the compact Higgs bundle spectral cover $\mathcal{C}_{l o c}$ in $S U(5), S O(10)$, and $E_{6}$ models. Actually resolving our 4 -fold $\mathcal{Y}_{4}$ with $E_{6}$ singularities, however, requires two further blow-ups that we describe briefly.

First, we blow-up along $\delta_{23}=\delta_{34}=0$ to get the 5 -times blown-up space $\mathcal{W}_{5}^{(5)}$. This gives an exceptional divisor $E_{5}$. The sections $\delta_{23}$ and $\delta_{34}$ factor in $\mathcal{W}_{5}^{(5)}$ as

$$
\begin{aligned}
& \delta_{23}=\delta_{235} \delta_{5} \\
& \delta_{34}=\delta_{345} \delta_{5}
\end{aligned}
$$

where the new objects are sections of the indicated bundles

\begin{tabular}{c|c} 
Section & Bundle \\
\hline$\delta_{235}$ & $\mathcal{O}\left(E_{2}-E_{3}-E_{5}\right)$ \\
$\delta_{345}$ & $\mathcal{O}\left(E_{3}-E_{4}-E_{5}\right)$ \\
$\delta_{5}$ & $\mathcal{O}\left(E_{5}\right)$
\end{tabular}

Finally, we blow-up along $\delta_{5}=\delta_{235}=0$ to get the 6 -times blown-up space $\mathcal{W}_{5}^{(6)}$. This gives an exceptional divisor $E_{6}$. The sections $\delta_{5}$ and $\delta_{235}$ factor in $\mathcal{W}_{5}^{(6)}$ as

$$
\begin{aligned}
\delta_{5} & =\delta_{56} \delta_{6} \\
\delta_{235} & =\delta_{2356} \delta_{6}
\end{aligned}
$$

where the new objects are sections of the indicated bundles

\begin{tabular}{c|c} 
Section & Bundle \\
\hline$\delta_{56}$ & $\mathcal{O}\left(E_{5}-E_{6}\right)$ \\
$\delta_{2356}$ & $\mathcal{O}\left(E_{2}-E_{3}-E_{5}-E_{6}\right)$ \\
$\delta_{6}$ & $\mathcal{O}\left(E_{6}\right)$
\end{tabular}




\section{Resolved Geometry}

At the end of our blow-ups we have the 5 -fold $\mathcal{W}_{5}^{(6)}$. Our original sections $x, y$, and $z$ factor in $\mathcal{W}_{5}^{(6)}$ according to

$$
\begin{aligned}
& x=x_{13} \delta_{12} \delta_{2356} \delta_{345}^{2} \delta_{4}^{2} \delta_{56}^{3} \delta_{6}^{4} \\
& y=y_{124} \delta_{12} \delta_{2356}^{2} \delta_{345}^{2} \delta_{4}^{3} \delta_{56}^{4} \delta_{6}^{6} \\
& z=z_{1} \delta_{12} \delta_{2356} \delta_{345} \delta_{4} \delta_{56}^{2} \delta_{6}^{3}
\end{aligned}
$$

For convenience, we summarize all of the relevant sections and their corresponding bundles as

\begin{tabular}{c|c} 
Section & Bundle \\
\hline$x_{13}$ & $\mathcal{O}\left(2[\sigma+\mathcal{D}]-E_{1}-E_{2}\right)$ \\
$y_{124}$ & $\mathcal{O}\left(3[\sigma+\mathcal{D}]-E_{1}-E_{2}-E_{4}\right)$ \\
$z_{1}$ & $\mathcal{O}\left(B_{2}-E_{1}\right)$ \\
$\delta_{12}$ & $\mathcal{O}\left(E_{1}-E_{2}\right)$ \\
$\delta_{2356}$ & $\mathcal{O}\left(E_{2}-E_{3}-E_{5}-E_{6}\right)$ \\
$\delta_{345}$ & $\mathcal{O}\left(E_{3}-E_{4}-E_{5}\right)$ \\
$\delta_{4}$ & $\mathcal{O}\left(E_{4}\right)$ \\
$\delta_{56}$ & $\mathcal{O}\left(E_{5}-E_{6}\right)$ \\
$\delta_{6}$ & $\mathcal{O}\left(E_{6}\right)$
\end{tabular}

We also give the Stanley-Reisner ideal

$$
\left\{\begin{array}{c}
v \delta_{12}, v \delta_{2356}, v \delta_{345}, v \delta_{4}, v \delta_{56}, v \delta_{6}, \\
\delta_{12} y_{124}, \delta_{12} \delta_{4}, \delta_{2356} x_{13}, \delta_{2356} \delta_{345}, \delta_{2356} \delta_{56}, \\
\delta_{345} z_{1}, \delta_{345} y_{124}, \delta_{345} \delta_{6}, \delta_{4} z_{1}, \delta_{56} x_{13}, \delta_{56} y_{124}, \delta_{56} z_{1}, \\
\delta_{6} x_{13}, \delta_{6} y_{124}, \delta_{6} z_{1}, z_{1} x_{13} y_{124}
\end{array}\right\}
$$

We now turn to the proper transform of $\tilde{\mathcal{Y}}_{4}$ of $\mathcal{Y}_{4}$ (A.2):

$$
\begin{array}{r}
\delta_{4}\left(\delta_{2356} y_{124}^{2}-\delta_{12} \delta_{345}^{2} \delta_{56} x_{13}^{3}-\delta_{12}^{3} \delta_{2356}^{2} \delta_{345}^{2} \delta_{56}^{3} \delta_{6}^{4} f x_{13}\left(z_{1} v\right)^{4}-\delta_{12}^{4} \delta_{2356}^{3} \delta_{345}^{2} \delta_{56}^{4} \delta_{6}^{6} g\left(z_{1} v\right)^{6}\right) \\
=\delta_{12} \delta_{2356} v^{3} z_{1}^{2}\left[b_{3} y_{124}+\delta_{12} \delta_{345} \delta_{56} \delta_{6} b_{2} x_{13}\left(z_{1} v\right)+\delta_{12}^{2} \delta_{2356} \delta_{345} \delta_{56}^{2} \delta_{6}^{3} b_{0}\left(z_{1} v\right)^{3}\right]
\end{array}
$$

This is smooth for generic $b_{m}$ and is in the class

$$
\tilde{\mathcal{Y}}_{4}=6[\sigma+\mathcal{D}]-2 E_{1}-E_{2}-E_{3}-E_{4}-E_{5}-E_{6}
$$

Since $c_{1}\left(\mathcal{W}_{5}^{(6)}\right)=c_{1}\left(\mathcal{W}_{5}\right)-2 E_{1}-E_{2}-E_{3}-E_{4}-E_{5}-E_{6}$ we have that

$$
c_{1}\left(\tilde{\mathcal{Y}}_{4}\right)=c_{1}\left(B_{3}\right)-\mathcal{D}
$$

indicating that the resolution is indeed crepant. It is a simple matter to describe the 
Cartan divisors of $\tilde{\mathcal{Y}}_{4}$, which are 3 -folds in $\mathcal{W}_{5}^{(6)}$

\begin{tabular}{c|c|c} 
Cart Div & Class in $\tilde{\mathcal{Y}}_{4}$ & Equations \\
\hline $\mathcal{D}_{1}$ & $E_{4}$ & $\delta_{4}=0$ \\
& $-\left(E_{2}-E_{3}-E_{5}-E_{6}\right)$ & $b_{3} y_{124}+\delta_{12} \delta_{345} \delta_{56} \delta_{6}\left(z_{1} v\right)\left(b_{2} x_{13}+b_{0} \delta_{12} \delta_{2356} \delta_{56} \delta_{6}^{2}\left(z_{1} v\right)^{2}\right)$ \\
& $+\left(E_{1}-E_{2}\right)$ & \\
\hline $\mathcal{D}_{2}$ & $\left(E_{2}-E_{3}-E_{5}-E_{6}\right)$ & $\delta_{2356}=0$ \\
& $-\left(E_{1}-E_{2}\right)$ & $\delta_{4}=0$ \\
\hline $\mathcal{D}_{3}$ & $E_{6}$ & $\delta_{6}=0$ \\
& & $\delta_{4}\left(\delta_{12} \delta_{345}^{2} \delta_{56} x_{13}^{3}-\delta_{2356} y_{124}^{2}\right)+v b_{3} \delta_{12} \delta_{2356} y_{124}\left(z_{1} v\right)^{2}=0$ \\
\hline $\mathcal{D}_{4}$ & $E_{5}-E_{6}$ & $\delta_{56}=0$ \\
& & $\delta_{4} y_{124}-v b_{3} \delta_{12}\left(z_{1} v\right)^{2}=0$ \\
\hline $\mathcal{D}_{5}$ & $E_{3}-E_{4}-E_{5}$ & $\delta_{345}=0$ \\
& & $\delta_{4} y_{124}-v b_{3} \delta_{12}\left(z_{1} v\right)^{2}=0$ \\
\hline $\mathcal{D}_{6}$ & $E_{1}-E_{2}$ & $\delta_{12}=0$ \\
& & $\delta_{2356}=0$
\end{tabular}

and of course we have the extended node

$$
\mathcal{D}_{0}=B_{2}-E_{1}
$$

To each Cartan divisor $\mathcal{D}_{i}$ we associate a curve $\Sigma_{i}$ which is the irreducible component of the singular fiber to which it corresponds. More specifically, $\Sigma_{i}$ is the intersection of $\mathcal{D}_{i}$ with $\pi^{*} p_{0}$ for a generic point $p_{0}$ in the section.

It is now a simple matter to compute the intersection matrix

\begin{tabular}{c|ccccccc}
$\tilde{\mathcal{Y}}_{4}$ & $\Sigma_{0}$ & $\Sigma_{1}$ & $\Sigma_{2}$ & $\Sigma_{3}$ & $\Sigma_{4}$ & $\Sigma_{5}$ & $\Sigma_{6}$ \\
$\mathcal{D}_{0}$ & -2 & 0 & 0 & 0 & 0 & 0 & 1 \\
$\mathcal{D}_{1}$ & 0 & -2 & 1 & 0 & 0 & 0 & 0 \\
$\mathcal{D}_{2}$ & 0 & 1 & -2 & 1 & 0 & 0 & 0 \\
$\mathcal{D}_{3}$ & 0 & 0 & 1 & -2 & 1 & 0 & 1 \\
$\mathcal{D}_{4}$ & 0 & 0 & 0 & 1 & -2 & 1 & 0 \\
$\mathcal{D}_{5}$ & 0 & 0 & 0 & 0 & 1 & -2 & 0 \\
$\mathcal{D}_{6}$ & 1 & 0 & 0 & 1 & 0 & 0 & -2
\end{tabular}

which is the intersection matrix of the extended $E_{6}$ Dynkin diagram as expected.

\section{A.2 Spectral Divisor, Heterotic Spectral Cover, and Higgs Bundle Spectral Cover}

We now describe the spectral divisor $\mathcal{C}_{F}$ in detail and its connection to the heterotic and Higgs bundle spectral covers, $\mathcal{C}_{H e t}$ and $\mathcal{C}_{\text {loc }}$. Among other things, we give a clear prescription for the compact surface $\mathcal{C}_{l o c}$ that emerges from the intersection of $\mathcal{C}_{F}$ with $\pi^{*} B_{2}$ and its equivalence to $\mathcal{C}_{H e t}$ when a heterotic dual exists. 


\section{The Spectral Divisor}

First, we look at the spectral divisor $\mathcal{C}_{F}$ (3.4). In $\mathcal{Y}_{4}$, it is the irreducible component of the hypersurface

$$
y^{2}=x^{3}+f x z^{4} v^{4}+g z^{6} v^{6}
$$

This surface is a 3 -sheeted cover of $B_{3}$ inside $\mathcal{Y}_{4}$ that is singular at $z=0$ where the sheets come together. The true object of interest is the proper transform $\tilde{\mathcal{C}}_{F}$ of $\mathcal{C}_{F}$ inside $\tilde{\mathcal{Y}}_{4}$ since it is here that we define the line bundle $\mathcal{N}_{F}$ for constructing $C_{3}$ and $G_{4}$. The 3 -fold $\tilde{\mathcal{C}}_{F}$ is smooth and can be most easily described as a complete intersection in $\mathcal{W}_{5}^{(6)}$

$$
\begin{aligned}
\delta_{2356} y_{124}^{2} & =\delta_{12} \delta_{345}^{2} \delta_{56}\left(x_{13}^{3}+\delta_{12}^{2} \delta_{2356}^{2} \delta_{56}^{2} \delta_{6}^{4} f x_{13}\left(z_{1} v\right)^{4}+\delta_{12}^{3} \delta_{2356}^{3} \delta_{56}^{3} \delta_{6}^{6} g\left(z_{1} v\right)^{6}\right) \\
0 & =b_{3} y_{124}+\delta_{12} \delta_{345} \delta_{56} \delta_{6} b_{2} x_{13}\left(z_{1} v\right)+\delta_{12}^{2} \delta_{2356} \delta_{345} \delta_{56}^{2} \delta_{6}^{3} b_{0}\left(z_{1} v\right)^{3}
\end{aligned}
$$

which is in the class

$$
\tilde{\mathcal{C}}_{F}=\left(3 \sigma+6 \mathcal{D}-E_{1}-E_{2}-E_{4}-2 B_{2}\right) \cdot\left(6 \sigma+6 \mathcal{D}-2 E_{1}-E_{2}-E_{3}-2 E_{4}-E_{5}-E_{6}\right)
$$

We can write the class of $\tilde{\mathcal{C}}_{F}$ as a restriction of a divisor class in $\mathcal{W}_{5}^{(6)}$ to $\tilde{\mathcal{Y}}_{4}$ using

$$
\begin{aligned}
{\left[b_{3} y_{124}+\ldots\right] \cdot\left[\tilde{\mathcal{Y}}_{4}\right] } & =\tilde{\mathcal{C}}_{F}+\left[b_{3} y_{124}+\ldots\right] \cdot \delta_{4} \\
{\left[\delta_{4}\right] \cdot\left[\tilde{\mathcal{Y}}_{4}\right] } & =\left[\delta_{4}\right] \cdot\left[b_{3} y_{124}+\ldots\right]+\left[\delta_{4}\right] \cdot\left[\delta_{2356}\right] \\
{\left[\delta_{2356}\right] \cdot\left[\tilde{\mathcal{Y}}_{4}\right] } & =\left[\delta_{2356}\right] \cdot\left[\delta_{4}\right]+\left[\delta_{2356}\right] \cdot\left[\delta_{12}\right] \\
{\left[\delta_{12}\right] \cdot\left[\tilde{\mathcal{Y}}_{4}\right] } & =\left[\delta_{12}\right] \cdot\left[\delta_{2356}\right]
\end{aligned}
$$

This means that

$$
\begin{aligned}
\tilde{\mathcal{C}}_{F} & =\left(\left[b_{3} y_{124}+\ldots\right]-\left[\delta_{4}\right]+\left[\delta_{2356}\right]-\left[\delta_{12}\right]\right) \cdot\left[\tilde{\mathcal{Y}}_{4}\right] \\
& =\left.\left(3 \sigma+6 \mathcal{D}-2 E_{1}+E_{2}-E_{3}-2 E_{4}-E_{5}-E_{6}-2 B_{2}\right)\right|_{\tilde{\mathcal{Y}}_{4}}
\end{aligned}
$$

It is also easy to verify that $\tilde{\mathcal{C}}_{F}$ has the expected intersections with the nodes $\Sigma_{i}$ of the $E_{6}$ singular fiber

$$
\begin{array}{c|ccccccc}
\cdot \tilde{\mathcal{Y}}_{4} & \Sigma_{0} & \Sigma_{1} & \Sigma_{2} & \Sigma_{3} & \Sigma_{4} & \Sigma_{5} & \Sigma_{6} \\
\hline \mathcal{\mathcal { C }}_{F} & 0 & 3 & 0 & 0 & 0 & 0 & 0
\end{array}
$$

Indeed, $\tilde{\mathcal{C}}_{F}$ is supposed to behave near $B_{2}$ as the cylinder of the heterotic/F-theory duality map in that it is a 3 -sheeted cover of $B_{2}$ whose sheets each intersect the $E_{6}$ singular fiber according to the highest weight of the $\mathbf{2 7}$ representation. The highest weight of the $\mathbf{2 7}$ is $(1,0,0,0,0,0)$ in our labeling conventions for the nodes $\Sigma_{i}(i=$ $1, \ldots, 6)$ so this works out correctly.

\section{The Higgs Bundle Spectral Cover, $\mathcal{C}_{l o c}$}

We now turn to the emergence of the Higgs bundle spectral cover, $\mathcal{C}_{\text {loc }}$, from $\mathcal{C}_{F}$ with special attention to describing the compact surface $\mathcal{C}_{\text {loc }}$ in its entirety. By $\mathcal{C}_{\text {loc }}$, we mean something very special: the restriction of $\mathcal{C}_{F}$ to $\pi^{*} B_{2}$ or, more properly, the restriction of $\tilde{\mathcal{C}}_{F}$ to $\pi^{*} B_{2}$. We saw before (A.54) that $\tilde{\mathcal{C}}_{F}$ intersects only one node of the $E_{6}$ singular 
fiber, namely the node $\Sigma_{1}$ corresponding to the Cartan divisor $\mathcal{D}_{1}=E_{4}$. The surface $\mathcal{C}_{\text {loc }}$, then, is obtained as the following intersection in $\mathcal{W}_{5}^{(6)}$

$$
\begin{aligned}
\mathcal{C}_{l o c} & =\tilde{\mathcal{C}}_{F} \cdot E_{4} \\
& =\left(3 \sigma+6 \mathcal{D}-E_{1}-E_{2}-E_{4}-2 B_{2}\right) \cdot\left(6 \sigma+6 \mathcal{D}-2 E_{1}-E_{2}-E_{3}-2 E_{4}-E_{5}-E_{6}\right) \cdot E_{4}
\end{aligned}
$$

which we describe in equations as

$\mathcal{C}_{l o c}:\left\{\begin{aligned} \delta_{2356} y_{124}^{2} & =\delta_{12} \delta_{345}^{2} \delta_{56}\left(x_{13}^{3}+\delta_{12}^{2} \delta_{2356}^{2} \delta_{56}^{2} \delta_{6}^{4} f x_{13}\left(z_{1} v\right)^{4}+\delta_{12}^{3} \delta_{2356}^{3} \delta_{56}^{3} \delta_{6}^{6} g\left(z_{1} v\right)^{6}\right) \\ 0 & =b_{3} y_{124}+\delta_{12} \delta_{345} \delta_{56} \delta_{6} b_{2} x_{13}\left(z_{1} v\right)+\delta_{12}^{2} \delta_{2356} \delta_{345} \delta_{56}^{2} \delta_{6}^{3} b_{0}\left(z_{1} v\right)^{3} \\ 0 & =\delta_{4}\end{aligned}\right.$

As written, this is a bit cumbersome to work with. We can make our lives easier, however, by considering the proper transform $\tilde{\mathcal{C}}_{F}^{(4)}$ of $\mathcal{C}_{F}$ under only the first 4 blow-ups of sections A.1.1 A.1.4. Consider then the surface

$$
\mathcal{C}_{l o c}^{(4)}=\tilde{\mathcal{C}}_{F}^{(4)} \cdot \mathcal{W}_{5}^{(4)} E_{4}
$$

given by the following complete intersection in $\mathcal{W}_{5}^{(4)}$

$$
\mathcal{C}_{l o c}^{(4)}:\left\{\begin{aligned}
\delta_{23} y_{124}^{2} & =\delta_{12} \delta_{34}^{2} x_{13}^{3}+\delta_{12}^{3} \delta_{23}^{2} \delta_{34}^{2} f x_{13}\left(z_{1} v\right)^{4}+\delta_{12}^{4} \delta_{23}^{3} \delta_{34}^{2} g\left(z_{1} v\right)^{6} \\
0 & =b_{3} y_{124}+b_{2} \delta_{12} \delta_{34} x_{13}\left(z_{1} v\right)+b_{0} \delta_{12}^{2} \delta_{23} \delta_{34}\left(z_{1} v\right)^{3} \\
0 & =\delta_{4}
\end{aligned}\right.
$$

The remaining two blow-ups are along 3-folds that meet $\mathcal{C}_{\text {loc }}^{(4)}$ along the curve $\delta_{23}=\delta_{34}=$ $b_{3}=\delta_{4}=0$. These are blow-ups in codimension 1 ; they have no effect on $\mathcal{C}_{\text {loc }}^{(4)}$ so it is isomorphic to $\mathcal{C}_{\text {loc }}$

$$
\mathcal{C}_{l o c}^{(4)}=\mathcal{C}_{l o c}
$$

We prefer to work with the description (A.58) of $\mathcal{C}_{l o c}$ inside $\mathcal{W}_{5}^{(4)}$ because it is a simple presentation of $\mathcal{C}_{l o c}$ as a divisor inside the exceptional divisor $E_{4}$ that we described in section A.1.4. Recall from that discussion that $E_{4}$ is an $\mathbb{F}_{1}$ fibration with homogeneous coordinates on the base given by restrictions of $\left[x_{13}, \delta_{23}\right]$ and homogeneous coordinates on the fiber given by restrictions of $\left[y_{124}, \delta_{34}\right]$. These were associated to bundles on $E_{4}$ as

\begin{tabular}{c|c} 
Section & Bundle on $E_{4}$ \\
\hline$x_{13}$ & $\mathcal{O}_{E_{4}}\left(\sigma_{3}+2 \mathcal{D}-B_{2}\right)$ \\
$\delta_{23}$ & $\mathcal{O}_{E_{4}}\left(\sigma_{3}+B_{2}\right)$ \\
$y_{124}$ & $\mathcal{O}_{E_{4}}\left(\sigma_{4}+3 \mathcal{D}-2 B_{2}\right)$ \\
$\delta_{34}$ & $\mathcal{O}_{E_{4}}\left(\sigma_{4}-\sigma_{3}\right)$
\end{tabular}

Let us introduce new notation to make things look simpler and clarify the structure of the $\mathbb{F}_{1}$ fibration. By direct computation, it is easy to verify that the curve $\delta_{34}=0$ inside the $\mathbb{F}_{1}$ has self-intersection -1. Renaming the classes $\sigma_{4}-\sigma_{3}=b$ and $\sigma_{3}+B_{2}=f$ and using $[W, X]$ for homogeneous coordinates on the base and $[u, q]$ for homogeneous 
coordinates on the fiber, we have

\begin{tabular}{c|c|c} 
Section & Restriction to $E_{4}$ & Bundle on $E_{4}$ \\
\hline$x_{13}$ & $X$ & $\mathcal{O}\left(f+2\left(\mathcal{D}-B_{2}\right)\right)$ \\
$\delta_{23}$ & $W$ & $\mathcal{O}(f)$ \\
$y_{124}$ & $q$ & $\mathcal{O}\left(b+f+3\left(\mathcal{D}-B_{2}\right)\right)$ \\
$\delta_{34}$ & $u$ & $\mathcal{O}(b)$
\end{tabular}

Finally, since $\delta_{12}=z_{1}=v=1$ everywhere along $E_{4}$, we can rewrite the first two equations of (A.58), which define $\mathcal{C}_{l o c}$ inside $E_{4}$, as

$$
\begin{aligned}
W q^{2} & =u^{2}\left(X^{3}+W^{2} X f+W^{3} g\right) \\
0 & =a_{3} q+a_{2} u X+a_{0} u W
\end{aligned}
$$

where we replaced the $b_{m}$ by $a_{m}$

$$
a_{m}=\left.b_{m}\right|_{B_{2}}
$$

which represent the restriction of $b_{m}$ to $\delta_{4}=0$. Above generic points in $B_{2}$ the first equation defines a curve in $\mathbb{F}_{1}$ in the class $2 b+3 f$. This is an anti-canonical curve and hence has genus 0 . We see, then, that the first equation defines an elliptic fibration $\mathcal{Z}_{3}$ over $B_{2}$. The second gives a 3 -fold cover of $B_{2}$ inside $\mathcal{Z}_{3}$. We can say more about $\mathcal{Z}_{3}$ and $\mathcal{C}_{\text {loc }}$, in fact. We have that

$$
c_{1}\left(E_{4}\right)=c_{1}\left(B_{2}\right)+2 b+3 f+5\left(\mathcal{D}-B_{2}\right)
$$

while $\mathcal{Z}_{3}$ is a hypersurface of $E_{4}$ in the class

$$
2 b+3 f+6\left(\mathcal{D}-B_{2}\right)
$$

This means that

$$
c_{1}\left(\mathcal{Z}_{3}\right)=c_{1}\left(B_{2}\right)-\left(\mathcal{D}-B_{2}\right)
$$

In all of the examples that we study in the rest of this Appendix (i.e. $\mathcal{Y}_{4}$ a Calabi-Yau 4 -fold or a $d P_{9}$-fibration), we will have that

$$
\left.\left(\mathcal{D}-B_{2}\right)\right|_{B_{2}}=c_{1}\left(B_{2}\right)
$$

so we make this assumption from now on. This means that $\mathcal{Z}_{3}$ is an elliptically fibered Calabi-Yau 3-fold

$$
c_{1}\left(\mathcal{Z}_{3}\right)=0
$$

In fact, we can use the replacements

$$
W=v^{2}, \quad X=x, \quad q=y, \quad u=v
$$

to set up a map from $\mathcal{Z}_{3}$ to a Calabi-Yau hypersurface of a $\mathbb{P}_{1,2,3}^{2}$-bundle $\mathcal{X}_{4}$ over $B_{2}$ with weighted homogeneous coordinates $[v, x, y]$ on the fiber. More specifically, $[v, x, y]$ are sections of the indicated bundles

\begin{tabular}{c|c} 
Section & Bundle \\
\hline$v$ & $\sigma_{l o c}$ \\
$x$ & $2\left(\sigma_{l o c}+c_{1, B_{2}}\right)$ \\
$y$ & $3\left(\sigma_{l o c}+c_{1, B_{2}}\right)$
\end{tabular}


while the 3 -fold $\mathcal{Z}_{3}$ is the hypersurface

$$
\mathcal{Z}_{3}: \quad y^{2}=x^{3}+f x v^{4}+g v^{6}
$$

and the final equation of (A.58) maps to

$$
0=a_{3} y+a_{2} v x+a_{0} v^{3}
$$

This is the conventional form of an $S U(3)$ spectral cover inside $\mathcal{Z}_{3}$. We recognize this as a specific compactification of the non-compact spectral cover of local models. The cover $\mathcal{C}_{l o c}$ can be viewed as a divisor inside the Calabi-Yau 3-fold $\mathcal{Z}_{3}$ in the class

$$
\mathcal{C}_{l o c}=3 \sigma_{l o c}+\eta_{l o c} \quad \eta_{l o c}=6 c_{1, B_{2}}+B_{2}
$$

where as usual we do not distinguish between divisors in the base, $B_{2}$, and their pullbacks to $\mathcal{Z}_{3}$.

The realization (A.72) of $\mathcal{C}_{\text {loc }}$ as a hypersurface inside the auxiliary elliptically fibered Calabi-Yau 3-fold $\mathcal{Z}_{3}$ (A.71) is very useful for calculations. Let us describe how restrictions of divisors on $\tilde{\mathcal{Y}}_{4}$ appear in this setting. Following through our studies of $E_{4}$ in section A.1.4, it is easy to see that

$$
\left.E_{1}\right|_{E_{4}}=\left.E_{2}\right|_{E_{4}}=\left.B_{2} \quad \Longrightarrow E_{1}\right|_{\mathcal{C}_{l o c}}=\left.E_{2}\right|_{\mathcal{C}_{l o c}}=B_{2}
$$

The map A.69) now tells us that

$$
\left.E_{3}\right|_{E_{4}}=-\left.\left(2 \sigma_{l o c}-B_{2}\right) \quad E_{4}\right|_{E_{4}}=-\left(3 \sigma_{l o c}-B_{2}\right)
$$

As a quick check, we can use this dictionary to compute $c_{1}\left(\mathcal{C}_{l o c}\right)$

$$
\begin{aligned}
c_{1}\left(\mathcal{C}_{l o c}\right)= & \left.c_{1}\left(\mathcal{W}_{5}^{(6)}\right)\right|_{\mathcal{C}_{l o c}} \\
& -\left.\left[\left(3 \sigma+6 \mathcal{D}-E_{1}-E_{2}-E_{4}-2 B_{2}\right)+\left(6 \sigma+6 \mathcal{D}-2 E_{1}-E_{2}-E_{3}-2 E_{4}-E_{5}-E_{6}\right)+\left(E_{4}\right)\right]\right|_{\mathcal{C}_{l o c}} \\
= & -\left.\left[3 \sigma+7 \mathcal{D}-c_{1, B_{3}}-E_{1}-E_{2}-E_{4}-2 B_{2}\right]\right|_{\mathcal{C}_{l o c}} \\
= & -\left.\left(3 \sigma_{l o c}+\eta_{l o c}\right)\right|_{\mathcal{C}_{l o c}}
\end{aligned}
$$

where we have expressed $c_{1}\left(\mathcal{C}_{\text {loc }}\right)$ as the restriction of divisor classes in $\mathcal{Z}_{3}$ in the last line using (A.74) (A.75) along with the trivial statement $\left.\sigma\right|_{E_{4}}=0$ and the property (A.67) of $\mathcal{D}$. This result is exactly what we expect because it is simply $c_{1}\left(\mathcal{C}_{\text {loc }}\right)=-\left.\left[\mathcal{C}_{\text {loc }}\right]\right|_{\mathcal{C}_{\text {loc }}}$ where $\left[\mathcal{C}_{l o c}\right]$ is the class of $\mathcal{C}_{l o c}$ as a divisor inside the Calabi-Yau 3-fold $\mathcal{Z}_{3}$.

The relations (A.75) are particularly useful for constructing divisors $\gamma_{F}$ on $\tilde{\mathcal{C}}_{F}$ that 'extend' divisors on $\mathcal{C}_{l o c}$ for building $G$-flux. Consider, for instance, the standard inherited local model bundle

$$
\mathcal{N}_{l o c}=\mathcal{O}_{\mathcal{C}_{l o c}}\left(\lambda \gamma_{l o c}+r_{l o c} / 2\right)
$$

where $r_{l o c}$ is the ramification divisor of the covering $p_{l o c}: \mathcal{C}_{l o c} \rightarrow B_{2}$ and $\gamma_{l o c}$ is the divisor class

$$
\gamma_{l o c}=3 \sigma_{l o c}-\left(\eta_{l o c}-3 c_{1, B_{2}}\right)=3 \sigma_{l o c}-\left(3 c_{1, B_{2}}-t\right)
$$

Here $t$ is the usual shorthand notation for divisor associated to the inverse normal bundle

$$
\mathcal{O}_{B_{2}}(-t)=N_{B_{2} / B_{3}}=\left.\mathcal{O}_{B_{3}}\left(B_{2}\right)\right|_{B_{2}}
$$


When we construct the $G$-flux corresponding to $\mathcal{N}_{\text {loc }}$, part of our task is to find a divisor $\gamma_{F}$ that satisfies

$$
\left.\gamma_{F}\right|_{\mathcal{C}_{l o c}}=\gamma_{l o c}
$$

We can do this by noting that

$$
\eta-3 c_{1, B_{2}}=\left.\left[3 c_{1, B_{3}}-4 B_{2}\right]\right|_{B_{2}}
$$

and using (A.75) to make the replacement

$$
\sigma_{l o c} \rightarrow E_{3}-E_{4}
$$

In this way, we arrive at

$$
\gamma_{F}=\left.\left[3\left(E_{3}-E_{4}\right)-\left(3 c_{1, B_{3}}-2 B_{2}\right)\right]\right|_{\tilde{\mathcal{C}}_{F}}
$$

which is an important building block for the $C_{3} / G_{4}$ configuration corresponding to the local model data (A.77).

We close this section by looking at another useful quantity, namely the normal bundle of $\mathcal{C}_{\text {loc }}$ inside $\tilde{\mathcal{C}}_{F}$

$$
N_{\mathcal{C}_{l o c} / \tilde{\mathcal{C}}_{F}}=\left.\mathcal{O}\left(E_{4}\right)\right|_{\mathcal{C}_{l o c}}=\left.\mathcal{O}_{\mathcal{Z}_{3}}\left(B_{2}-3 \sigma_{l o c}\right)\right|_{\mathcal{C}_{l o c}}
$$

This allows us to relate the ramification divisor $r_{l o c}$ of the covering $p_{l o c}: \mathcal{C}_{l o c} \rightarrow B_{2}$ to the restriction of the ramification divisor $r_{F}$ of $p_{F}: \tilde{\mathcal{C}}_{F} \rightarrow B_{3}$

$$
\begin{aligned}
\left.r_{F}\right|_{\mathcal{C}_{l o c}} & =\left[p_{F}^{*} c_{1, B_{3}}-c_{1}\left(\tilde{\mathcal{C}}_{F}\right)\right]_{\mathcal{C}_{l o c}} \\
& =p_{l o c}^{*}\left(c_{1, B_{2}}+B_{2}\right)-c_{1}\left(\mathcal{C}_{l o c}\right)-N_{\mathcal{C}_{l o c} / \tilde{\mathcal{C}}_{F}} \\
& =r_{l o c}+3 \sigma_{l o c}
\end{aligned}
$$

In light of this, let us define a bundle $\mathcal{L}_{\hat{r}}$ on $\tilde{\mathcal{C}}_{F}$ by

$$
\mathcal{L}_{\hat{r}}=\left.\mathcal{O}_{\tilde{\mathcal{C}}_{F}}\left(r_{F}\right) \otimes \mathcal{O}\left(-3\left[E_{3}-E_{4}\right]\right)\right|_{\mathcal{C}_{l o c}}
$$

We have that

$$
\left.\mathcal{L}_{\hat{r}}\right|_{\mathcal{C}_{l o c}}=\mathcal{O}\left(r_{l o c}\right)
$$

which will be helpful in our later discussion of flux quantization.

\section{$\mathcal{Y}_{4}$ a Calabi-Yau and $G$-flux Quantization}

We now look at a few special choices for the class $\mathcal{D}$ in (A.1). To apply the results of this Appendix to $\mathcal{Y}_{4}$ 's that are Calabi-Yau, we simply set

$$
\mathcal{D}=c_{1, B_{3}}
$$

Our object $\tilde{\mathcal{C}}_{F}$ (A.50) is a generic spectral divisor of the Calabi-Yau 4-fold $\tilde{Y}_{4}$ and its intersection with $E_{4}$ yields a compactification of the local model Higgs bundle spectral cover obtained by embedding the total space of $K_{S_{2}}$ into the auxiliary Calabi-Yau 3-fold $\mathcal{Z}_{3}$. Note that there is some ambiguity in the choice of $\tilde{\mathcal{C}}_{F}$ because we could absorb 
part or all of the $f$ and $g$ terms in (A.43) into the $b_{m}$ 's. If we absorb them completely, effectively setting $f$ and $g$ to zero in (A.43), then we obtain the analog of the 'Tate divisor' of [28, 30, 41] which can extend a factorization structure of the Higgs bundle spectral cover. We do not consider split spectral covers here so there is no difference between the analog of the 'Tate divisor' or any other spectral divisor.

We devote the rest of this subsection to the $G$-flux quantization rule (3.5) [70]

$$
G_{4}+\frac{1}{2} c_{2}\left(\tilde{\mathcal{Y}}_{4}\right) \in H^{2,2}\left(\tilde{Y}_{4}, \mathbb{Z}\right)
$$

Because of the related quantization rule in the local model that follows from (3.13)

$$
\lambda \gamma_{l o c}+\frac{r_{l o c}}{2} \in H_{2}\left(\mathcal{C}_{l o c}, \mathbb{Z}\right)
$$

and the general expectation that $\lambda \gamma_{l o c}$ maps to $G$-flux, we expect that the odd part of $c_{2}\left(\tilde{Y}_{4}\right)$ is captured by $\iota_{F *} c_{1}\left(\mathcal{L}_{\hat{r}}\right)$ for some line bundle $\mathcal{L}_{\hat{r}}$ on $\tilde{\mathcal{C}}_{F}$ such that $\left.\mathcal{L}_{\hat{r}}\right|_{\mathcal{C}_{\text {loc }}}=$ $\mathcal{O}_{\mathcal{C}_{l o c}}\left(r_{l o c}\right)$. We have already found such a bundle (A.86)

$$
\mathcal{L}_{\hat{r}}=\left.\left.\mathcal{O}_{\tilde{\mathcal{C}}_{F}}\left(r_{F}\right) \otimes \mathcal{O}\left(-3\left[E_{3}-E_{4}\right]\right)\right|_{\tilde{\mathcal{C}}_{F}} \quad \mathcal{L}_{\hat{r}}\right|_{\mathcal{C}_{l o c}}=\mathcal{O}_{\mathcal{C}_{l o c}}\left(r_{l o c}\right)
$$

One might conjecture, then, that

$$
c_{2}\left(\tilde{Y}_{4}\right)+\iota_{F *} c_{1}\left(\mathcal{L}_{\hat{r}}\right) \quad \text { is an even class? }
$$

It turns out that this is almost true but not quite. To investigate further, we must compute $c_{2}\left(\tilde{Y}_{4}\right)$ explicitly. The computation is straightforward [32] but messy and can be complicated to interpret because the result is expressed as a linear combination of surfaces of the form

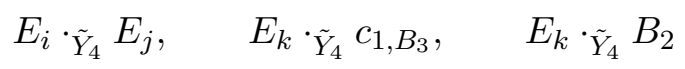

which, as in [32], is very redundant. We can use the intersection relations that follow from (A.42) to relate several elements of (A.93) to one another. Further, there are additional intersection relations in $\tilde{Y}_{4}$ that are not the restriction of relations in the ambient 5 -fold. We phrase these as another collection of monomials as in (A.42) corresponding to holomorphic sections that do not simultaneously vanish in $\tilde{Y}_{4}$

$$
\left\{\delta_{12} x_{13}, \delta_{12} \delta_{345}, \delta_{12} \delta_{56}, z_{1} y_{124}, z_{1} x_{13}\right\}
$$

The fact that $z_{1}=y_{124}=0$ and $z_{1}=x_{13}=0$ have no solutions in $\tilde{\mathcal{Y}}_{4}$ means that

$$
\left(E_{1}-2 E_{2}+3 E_{3}-2 E_{4}\right) \cdot \tilde{\mathcal{Y}}_{4}\left(B_{2}-E_{1}\right)=0
$$

Using the relations from (A.42) and (A.94), then, we get a set 19 relations that can be used to relate 19 of the 21 surfaces of the form

$$
E_{i} \cdot \tilde{\mathcal{Y}}_{4} E_{j}
$$

to surfaces of the form

$$
E_{i} \cdot \tilde{Y}_{4} c_{1, B_{3}}, \quad E_{i} \cdot \tilde{Y}_{4} B_{2}, \quad E_{1} \cdot_{\tilde{Y}_{4}} E_{2}, \quad E_{3} \cdot \tilde{Y}_{4} E_{4}
$$


The 19 equations that we choose to apply are as follows:

$$
\begin{aligned}
E_{1}^{2} & =B_{2}\left(E_{1}-2 E_{2}\right)+2 E_{1} E_{2} \\
E_{2}^{2} & =B_{2}\left(E_{1}-2 E_{2}\right)+3 c_{1, B_{3}}\left(E_{2}-E_{1}\right)+2 E_{1} E_{2} \\
E_{3}^{2} & =B_{2}\left(E_{1}-2 E_{2}\right)+c_{1, B_{3}}\left(-3 E_{1}+E_{2}+2 E_{3}\right)+2 E_{1} E_{2} \\
E_{4}^{2} & =B_{2}\left(E_{1}-2 E_{2}+2 E_{3}-2 E_{4}\right)+3 c_{1, B_{3}}\left(-E_{1}+E_{2}-E_{3}+E_{4}\right)+E_{1} E_{2}+E_{3} E_{4} \\
E_{5}^{2} & =B_{2}\left(2 E_{1}-4 E_{2}-E_{3}+E_{4}+3 E_{5}\right)+c_{1, B_{3}}\left(-6 E_{1}+4 E_{2}+2 E_{3}-3 E_{5}\right)+3 E_{1} E_{2}-E_{3} E_{4} \\
E_{6}^{2} & =B_{2}\left(2 E_{1}-4 E_{2}-E_{3}+E_{4}+E_{5}+2 E_{6}\right)+c_{1, B_{3}}\left(-6 E_{1}+4 E_{2}+2 E_{3}-E_{5}-2 E_{6}\right)+3 E_{1} E_{2}-E_{3} E_{4} \\
E_{1} E_{3} & =B_{2} E_{3} \\
E_{1} E_{4} & =B_{2} E_{4} \\
E_{1} E_{5} & =B_{2} E_{5} \\
E_{1} E_{6} & =B_{2} E_{6} \\
E_{2} E_{3} & =B_{2}\left(E_{1}-2 E_{2}+E_{3}\right)+3 c_{1, B_{3}}\left(E_{2}-E_{1}\right)+E_{1} E_{2} \\
E_{2} E_{4} & =B_{2} E_{4} \\
E_{2} E_{5} & =B_{2}\left(E_{1}-2 E_{2}+E_{5}\right)+3 c_{1, B_{3}}\left(E_{2}-E_{1}\right)+E_{1} E_{2} \\
E_{2} E_{6} & =B_{2}\left(E_{1}-2 E_{2}+E_{6}\right)+3 c_{1, B_{3}}\left(E_{2}-E_{1}\right)+E_{1} E_{2} \\
E_{3} E_{5} & =-B_{2} E_{5}+2 c_{1, B_{3}} E_{5} \\
E_{3} E_{6} & =-B_{2} E_{6}+2 c_{1, B_{3}} E_{6} \\
E_{4} E_{5} & =B_{2}\left(-E_{1}+2 E_{2}-2 E_{5}\right)+3 c_{1, B_{3}}\left(E_{1}-E_{2}+E_{5}\right)-E_{1} E_{2} \\
E_{4} E_{6} & =B_{2}\left(-E_{1}+2 E_{2}-2 E_{6}\right)+3 c_{1, B_{3}}\left(E_{1}-E_{2}+E_{6}\right)-E_{1} E_{2} \\
E_{5} E_{6} & =B_{2}\left(E_{1}-2 E_{2}+E_{6}\right)+c_{1, B_{3}}\left(-3 E_{1}+3 E_{2}-E_{6}\right)+E_{1} E_{2}
\end{aligned}
$$

where all of the intersections here are taken in $\tilde{Y}_{4}$. With these results, we can write $c_{2}\left(\tilde{Y}_{4}\right)$ as

$$
\begin{aligned}
c_{2}\left(\tilde{Y}_{4}\right) & =c_{2}\left(Y_{4}\right)-3 E_{1} E_{2}+3 E_{3} E_{4}-c_{1, B_{3}}\left(4 E_{1}+7 E_{3}+6 E_{4}+2 E_{6}\right) \\
& +B_{2}\left(2 E_{2}+3 E_{3}+E_{4}-E_{5}+E_{6}\right) \\
& =E_{1} E_{2}+E_{3} E_{4}+c_{1, B_{3}} E_{3}+B_{2}\left(E_{3}+E_{4}+E_{5}+E_{6}\right)+\text { even }
\end{aligned}
$$

This is to be compared with

$$
\begin{aligned}
\iota_{F *} c_{1}\left(\mathcal{L}_{\hat{r}}\right) & =\mathcal{C}_{F} \cdot \tilde{Y}_{4}\left(c_{1, B_{3}}+\tilde{\mathcal{C}}_{F}-3\left(E_{3}-E_{4}\right)\right) \\
& =E_{1} E_{2}+E_{3} E_{4}+c_{1, B_{3}}\left(E_{2}+E_{3}\right)+B_{2}\left(E_{3}+E_{4}+E_{5}+E_{6}\right)+\text { even }
\end{aligned}
$$

This means that (A.92) must be modified to

$$
c_{2}\left(\tilde{Y}_{4}\right)+c_{1}\left(\hat{\mathcal{L}}_{\hat{r}}\right)+G_{0} \text { is an even class }
$$

where $G_{0}$ is a 'Cartan correction term' of the form

$$
G_{0}=c_{1} \cdot \tilde{Y}_{4} E_{2}
$$


The presence of the Cartan correction term in (A.101) is actually very important for proper functioning of the local-to-global dictionary. Let us return to the local model bundle (A.77) with $\gamma_{l o c}$ as in (A.78). We found a divisor $\gamma_{F}$ (A.83) in $\tilde{C C}_{F}$

$$
\gamma_{F}=\left.\left[3\left(E_{3}-E_{4}\right)-\left(3 c_{1, B_{3}}-2 B_{2}\right)\right]\right|_{\tilde{\mathcal{C}}_{F}}
$$

with $\left.\gamma_{F}\right|_{\mathcal{C}_{l o c}}=\gamma_{l o c}$. This gives a holomorphic surface inside $\tilde{Y}_{4}$ whose Poincare dual specifies part of the $G$-flux. We must supplement this by two types of corrections, though. First, we must add horizontal and vertical surfaces (that is surfaces containing the elliptic fiber or sitting in the section of $\tilde{Y}_{4}$ ) to ensure that the resulting $G$-flux satisfies the 'one leg on the fiber' condition. Second, we have to add a Cartan flux so that the $G$-flux does not break $E_{6}$. We illustrate these corrections on the second and third lines of the following

$$
\begin{aligned}
\gamma_{l o c} \rightarrow \mathcal{G}= & {\left[3\left(E_{3}-E_{4}\right)-\left(3 c_{1, B_{3}}-2 B_{2}\right)\right] \tilde{Y}_{4} \tilde{\mathcal{C}}_{F} } \\
& +\left(3 c_{1, B_{3}}-2 B_{2}\right) \cdot_{\tilde{Y}_{4}}\left(3 \sigma+6 c_{1, B_{3}}-2 B_{2}\right) \\
& +E_{2} \tilde{Y}_{4}\left(6 S_{2}-9 c_{1, B_{3}}\right)
\end{aligned}
$$

In the end, it is not $\gamma_{l o c}$ that appears in $\mathcal{N}_{l o c}$ but a multiple $\lambda \gamma_{l o c}$ with the integrality or non-integrality of $\lambda$ fixed by the quantization condition (A.90). Similarly, the $G$-flux is $\lambda \mathcal{G}$ where we expect

$$
\lambda \mathcal{G}+\frac{1}{2} c_{2}\left(\tilde{Y}_{4}\right) \in H^{2,2}\left(\tilde{Y}_{4}, \mathbb{Z}\right)
$$

As is familiar for this type of flux, the local model result is that $\lambda$ must be an odd half-integer. For $\mathcal{G}$ to be properly quantized, then, it must be that the odd parts of $\mathcal{G}$ and $c_{2}\left(\tilde{Y}_{4}\right)$ agree. Because the first two lines of (A.104) were constructed directly from $\gamma_{l o c}$ we expect that the odd part of those lines is the same as the odd part of $\iota_{F *} c_{1}\left(\mathcal{L}_{\hat{r}}\right)$. Indeed, it is easy to check that

$$
\begin{aligned}
{\left[3\left(E_{3}-E_{4}\right)-\left(3 c_{1, B_{3}}-2 B_{2}\right)\right] \tilde{Y}_{4} \tilde{\mathcal{C}}_{F} } & +\left(3 c_{1, B_{3}}-2 B_{2}\right) \cdot \tilde{Y}_{4}\left(3 \sigma+6 c_{1, B_{3}}-2 B_{2}\right) \\
& +\tilde{\mathcal{C}}_{F} \cdot \tilde{Y}_{4} c_{1}\left(\hat{\mathcal{L}}_{\hat{r}}\right) \text { is an even class }
\end{aligned}
$$

If we did not have to add the Cartan flux on the third line of (A.104) then $\mathcal{G}$ would be properly quantized under the assumption (A.92) that $\iota_{F *} c_{1}\left(\mathcal{L}_{\hat{r}}\right)$ captures the full odd contribution to $c_{2}\left(\tilde{Y}_{4}\right)$. There is a Cartan correction (A.102) in the odd part of $c_{2}\left(\tilde{Y}_{4}\right)$ (A.101), though. This exactly matches the odd part of the third line in (A.104) that we had to add in order to make $\mathcal{G}$ truly $E_{6}$-preserving.

We should now summarize the construction of $G$-fluxes using the spectral divisor. We start with a line bundle $\mathcal{N}_{F}$ on $\tilde{\mathcal{C}}_{F}$. Let $A_{F}$ be the connection on this bundle and let $A_{\hat{r}}$ be the connection on $\mathcal{L}_{\hat{r}}$ (A.86). We then let

$$
C_{3}=\iota_{F *}\left(A_{F}-\frac{1}{2} A_{\hat{r}}\right)+\frac{1}{2} C_{3,0} \quad \leftrightarrow \quad G_{4} \sim \iota_{F *}\left(c_{1}\left(\mathcal{N}_{F}\right)-\frac{1}{2} \mathcal{L}_{\hat{r}}\right)+\frac{1}{2} G_{4,0}
$$

where

$$
G_{4,0}=c_{1, B_{3}} \cdot \tilde{Y}_{4} E_{2}
$$


and $C_{3,0}$ is a 3 -form with $d C_{3,0}=G_{4,0}$. We could let $A_{K^{-1}}$ be a connection on the anticanonical bundle of $B_{3}$ and take $C_{3,0}=A_{K^{-1}} \wedge c_{1}\left(\mathcal{O}\left(E_{2}\right)\right)$ where we implicitly mean that $A_{K^{-1}}$ should be pulled back to $\tilde{Y}_{4}$.

There is no guarantee that this procedure will yield an $E_{6}$-preserving $G$-flux. In these cases, one can try to restore $E_{6}$ by adding suitable integrally quantized Cartan fluxes. The $G$-flux in (A.104) is an example of this.

\section{$\mathcal{Y}_{4}$ a $d P_{9}$ fibration and the Cylinder}

This is the case of interest for the stable degeneration limit of section 4.1. Here, we take $B_{3}$ to be a $\mathbb{P}^{1}$-fibration over $B_{2}$ obtained by projectivizing a line bundle $N$ (4.1)

$$
B_{3}=\mathbb{P}(\mathcal{O} \oplus N)
$$

Following the main text, we use the notation $s$ for the divisor class of a section of this $\mathbb{P}^{1}$ bundle and let $[Z, W]$ denote homogeneous coordinates on the fiber

\begin{tabular}{c|c} 
Section & Bundle \\
\hline$Z$ & $\mathcal{O}(s)$ \\
$W$ & $\mathcal{O}(s+t)$
\end{tabular}

with $t$ a divisor on $B_{2}$. With this notation, the surface of $E_{6}$ singularities is denoted by $s$ rather than $B_{2}$ to distinguish it from the other section, $s+t$, of $B_{3}$ which is also a copy of $B_{2}$. We recall that

$$
\left.c_{1, B_{3}}\right|_{B_{2}}=c_{1, B_{2}}+2 s+t
$$

We can obtain the $d P_{9}$-fibration $Y_{4}^{\prime}$ (4.3) from the general form (A.2) by choosing

$$
\mathcal{D}=s+c_{1, B_{2}}
$$

so that our initial sections are associated to the bundles

\begin{tabular}{c|c} 
Section & Bundle \\
\hline$v$ & $\mathcal{O}(\sigma)$ \\
$x$ & $\mathcal{O}\left(2\left[\sigma+s+c_{1, B_{2}}\right]\right)$ \\
$y$ & $\mathcal{O}\left(3\left[\sigma+s+c_{1, B_{2}}\right]\right)$ \\
$b_{m}$ & $\mathcal{O}\left(s+[6-m] c_{1, B_{2}}\right)$ \\
$f$ & $\mathcal{O}\left(4 c_{1, B_{2}}\right)$ \\
$g$ & $\mathcal{O}\left(6 c_{1, B_{2}}\right)$
\end{tabular}

We further write

$$
b_{m}=a_{m} W
$$

with

\begin{tabular}{c|c} 
Section & Bundle \\
\hline$a_{0}$ & $6 c_{1, B_{2}}-t=\eta$ \\
$a_{2}$ & $4 c_{1, B_{2}}-t=\eta-2 c_{1, B_{2}}$ \\
$a_{3}$ & $3 c_{1, B_{2}}-t=\eta-3 c_{1, B_{2}}$
\end{tabular}

where we have defined

$$
\eta \equiv 6 c_{1, B_{2}}-t
$$


With this notation, $\mathcal{Y}_{4}\left(\right.$ A.2) is the $d P_{9}$-fibration (4.3) from the text

$$
y^{2}=x^{3}+f x Z^{4} v^{4}+g Z^{6} v^{6}+Z^{2} W v^{3}\left[a_{0} Z^{3} v^{3}+a_{2} Z v x+a_{3} y\right]
$$

The restriction to $W=0$ yields the heterotic 3 -fold

$$
\mathcal{Z}_{H e t}: y^{2}=x^{3}+f x+g
$$

Our construction of the spectral divisor $\mathcal{C}_{F}$ goes through almost as in section A.2.1. We just have to make one subtle change. Because we chose the $b_{m}$ 's to have $W=0$ as a common factor (A.114), we must remove this common factor from the second equation in (A.50). The resulting 3-fold is the proper transform $\tilde{\mathcal{C}}_{c y l}$ of the cylinder (4.6)

$\tilde{\mathcal{C}}_{c y l}: \quad\left\{\begin{array}{l}0=\delta_{2356} y_{124}^{2}-\delta_{12} \delta_{345}^{2} \delta_{56} x_{13}^{3}-\delta_{12}^{3} \delta_{2356}^{2} \delta_{345}^{2} \delta_{56}^{3} \delta_{6}^{4} f x_{13}\left(Z_{1} v\right)^{4}-\delta_{12}^{4} \delta_{2356}^{3} \delta_{345}^{2} \delta_{56}^{4} \delta_{6}^{6} g\left(Z_{1} v\right)^{6} \\ 0=a_{3} y_{124}+a_{2} \delta_{12} \delta_{345} \delta_{56} \delta_{6} x_{13}\left(Z_{1} v\right)+a_{0} \delta_{12}^{2} \delta_{2356} \delta_{345} \delta_{56}^{2} \delta_{6}^{3}\left(Z_{1} v\right)^{3}\end{array}\right.$

which is the complete intersection

$$
\begin{aligned}
& \tilde{\mathcal{C}}_{c y l}=(\left(3 \sigma+\left(6\left(c_{1, B_{2}}-t\right)-E_{1}-E_{2}-E_{4}+3 s\right)\right. \\
& \cdot\left(6\left[\sigma+c_{1, B_{2}}+s\right]-2 E_{1}-E_{2}-E_{3}-2 E_{4}-E_{5}-E_{6}\right)
\end{aligned}
$$

in $\mathcal{W}_{5}^{(6)}$. The restriction of $\tilde{\mathcal{C}}_{c y l}$ to $\mathcal{Z}_{\text {Het }}$ is the heterotic spectral cover

$$
\mathcal{C}_{\text {Het }}: a_{0} v^{3}+a_{2} v x+a_{3} y=0
$$

In fact $\tilde{\mathcal{C}}_{c y l}$ is a $\mathbb{P}^{1}$-fibration over $\mathcal{C}_{H e t}$ with $\left[W, \delta_{4}\right]$ providing homogeneous coordinates on the $\mathbb{P}^{1}$ fiber. As usual for such a vibration, $\tilde{\mathcal{C}}_{c y l}$ admits two sections $W=0$ and $\delta_{4}=0$. The first is $\mathcal{C}_{\text {Het }}$ at $W=0$ while we know from section A.2.2 that the second, $\delta_{4}=0$, is the Higgs bundle spectral cover $\mathcal{C}_{l o c}$ (A.56). Consistency requires that $\mathcal{C}_{\text {loc }}$ be isomorphic to $\mathcal{C}_{\mathrm{Het}}$ and indeed this is easy to see from the discussion of section A.2.2. There, we showed that $\mathcal{C}_{\text {loc }}$ could be described as a surface inside an elliptically fibered Calabi-Yau 3-fold $\mathcal{Z}_{3}$. The identification (A.71) implies that

$$
\mathcal{Z}_{3}=\mathcal{Z}_{\text {Het }}
$$

and the defining equation of $\mathcal{C}_{\text {loc }}$ inside $\mathcal{Z}_{3}$ (A.72) is identical to A.121). We will continue to use the notation $\mathcal{C}_{H e t}$ for the section of $\tilde{\mathcal{C}}_{c y l}$ at $W=0$ and $\mathcal{C}_{\text {loc }}$ for the section at $\delta_{4}=0$.

We can describe the geometry of $\tilde{\mathcal{C}}_{c y l}$ in even more detail by providing the normal bundles of $\mathcal{C}_{\text {loc }}$ and $\mathcal{C}_{\text {Het }}$ in $\tilde{\mathcal{C}}_{c y l}$. The normal bundle of $\mathcal{C}_{\text {loc }}$ is captured by our computation in section A.2.2

$$
N_{\mathcal{C}_{l o c} / \tilde{\mathcal{C}}_{c y l}}=\left.\mathcal{O}_{\mathcal{Z}_{3}}\left(-t-3 \sigma_{l o c}\right)\right|_{\mathcal{C}_{l o c}}
$$

where we have replaced $B_{2}$ in (A.84) with $-t=\left.s\right|_{s}$. The normal bundle of $\mathcal{C}_{\text {Het }}$ is just the restriction of the normal bundle of $\mathcal{Z}_{H e t}$ inside $\tilde{\mathcal{Y}}_{4}$. Recalling that $\mathcal{Z}_{H e t}$ is just given by $W=0$, this is the bundle pulled back from $\left.\mathcal{O}_{B_{3}}(s+t)\right|_{s+t}=\left.\mathcal{O}_{B_{3}}(t)\right|_{s+t}$

$$
N_{\mathcal{C}_{H e t} / \tilde{\mathcal{C}}_{c y l}}=\left.\mathcal{O}_{\mathcal{Z}_{H e t}}(t)\right|_{\mathcal{C}_{H e t}}
$$


In the end, then, $\mathcal{C}_{c y l}$ is the following $\mathbb{P}^{1}$-fibration with base $\mathcal{C}_{H e t}$

$$
\mathcal{C}_{c y l}=\mathbb{P}_{\mathcal{C}_{H e t}}\left(\mathcal{O}_{\mathcal{C}_{H e t}}(t) \oplus \mathcal{O}_{\mathcal{C}_{H e t}}\left(-t-3 \sigma_{H e t}\right)\right)
$$

Let us turn now to ramification divisor and the bundle $\mathcal{L}_{\hat{r}}$ of (A.86). Since the normal bundle of $\mathcal{C}_{H e t}$ in $\tilde{\mathcal{C}}_{c y l}$ is pulled back from $B_{3}$, the ramification divisor $r_{H e t}$ of the covering $p_{\text {Het }}: \mathcal{C}_{\text {Het }} \rightarrow B_{2}$ is the restriction of the ramification divisor $r_{c y l}$ of $p_{c y l}: \tilde{\mathcal{C}}_{c y l} \rightarrow B_{3}$ to $\mathcal{C}_{\text {Het }}$

$$
\begin{aligned}
r_{F} \mid \mathcal{C}_{H e t} & =\left[p_{c y l}^{*} c_{1, B_{3}}-c_{1}\left(\tilde{\mathcal{C}}_{c y l}\right)\right]_{\mathcal{C}_{H e t}} \\
& =p_{H e t}^{*}\left(c_{1, B_{2}}+[W=0]\right)-c_{1}\left(\mathcal{C}_{H e t}\right)-N_{\mathcal{C}_{H e t} / \tilde{\mathcal{C}}_{c y l}} \\
& =r_{H e t}
\end{aligned}
$$

Because $\left.\mathcal{O}\left(-3\left[E_{3}-E_{4}\right]\right)\right|_{W=0}=0$ we also have that the bundle $\mathcal{L}_{\hat{r}}$ from (A.86) restricts to $\mathcal{O}_{\mathcal{C}_{H e t}}\left(r_{H e t}\right)$

$$
\left.\mathcal{L}_{\hat{r}}\right|_{\mathcal{C}_{H e t}}=\mathcal{O}_{\mathcal{C}_{\text {Het }}}\left(r_{H e t}\right)
$$

while the correction term (A.108) restricts trivially 27 . This is important because we still have that $\mathcal{L}_{\hat{r}}$ restricts to $\mathcal{O}_{\mathcal{C}_{l o c}}\left(r_{l o c}\right)$

$$
\left.\mathcal{L}_{\hat{r}}\right|_{\mathcal{C}_{l o c}}=\mathcal{O}_{\mathcal{C}_{l o c}}\left(r_{l o c}\right)
$$

Because $\mathcal{L}_{\hat{r}}$ restricts to the same bundle $\mathcal{O}_{\mathcal{C}_{\text {Het }}}\left(r_{H e t}\right)=\mathcal{O}_{\mathcal{C}_{\text {loc }}}\left(r_{l o c}\right)$ in both sections, $\mathcal{C}_{\text {Het }}$ and $\mathcal{C}_{l o c}$, it must be the pullback

$$
\mathcal{L}_{\hat{r}}=p_{\text {cyl }}^{*} \mathcal{O}_{\mathcal{C}_{\text {Het }}}\left(r_{H e t}\right)
$$

We can now write the map (A.107) from spectral data to $C_{3} / G_{4}$ as follows

$C_{3} \sim \iota_{c y l *} p_{c y l}^{*}\left(A_{c y l}-\frac{1}{2} A_{\hat{r}}\right)+\frac{1}{2} C_{3,0} \quad \leftrightarrow \quad G_{4} \sim \iota_{c y l *} p_{c y l}^{*}\left(c_{1}\left(\mathcal{N}_{H e t}\right)-\frac{1}{2} c_{1}\left(\mathcal{L}_{\hat{r}}\right)\right)+\frac{1}{2} G_{4,0}$

Apart from the Cartan correction terms (A.108), this is the usual cylinder map in which $p_{c y l}$ is used to pull bundle data back from $\mathcal{C}_{H e t}$ to $\mathcal{C}_{c y l}$ and the embedding map $\iota_{c y l}$ is used to push this forward into $\tilde{Y}_{4}^{\prime}$. It is easy to verify that the Poincare dual holomorphic surface to $G_{4}$ is nothing other than

$$
c_{1}\left(\mathcal{N}_{H e t}\right)-\frac{1}{2} r_{H e t}=\lambda \gamma_{H e t}
$$

with $\gamma_{\text {Het }}$ as in (2.10). In general, we will need to add additional Cartan fluxes to (A.130) in order to ensure that $E_{6}$ is truly unbroken on the F-theory side as it is on the heterotic side.

\footnotetext{
${ }^{27}$ The twist of $\mathcal{L}_{\hat{r}}$ relative to the ramification divisor of $\mathcal{C}_{c y l}$ A.86) reflects the fact that the normal bundle of $\mathcal{C}_{\text {loc }}$ inside $\mathcal{C}_{c y l}$, or equivalently $\mathcal{C}_{F}$, is not pulled back from $B_{2}$. Had the normal bundle been a simple pullback from $B_{2}$, the ramification divisor of $\mathcal{C}_{c y l}$ would have been a simple pullback of the ramification divisor of $\mathcal{C}_{\text {loc }}$. Instead $\mathcal{L}_{\hat{r}}$, which is the pullback of $\mathcal{O}_{\mathcal{C}_{l o c}}\left(r_{l o c}\right)$, or equivalently $\mathcal{O}_{\mathcal{C}_{H e t}}\left(r_{H e t}\right)$, to $\mathcal{C}_{c y l}$, is twisted relative to $\mathcal{O}_{\mathcal{C}_{c y l}}\left(r_{c y l}\right)$ by the contribution to $N_{\mathcal{C}_{l o c} / \mathcal{C}_{c y l}}$ that is not pulled back from $B_{2}$.
} 


\section{B Details of the Base in Example Two}

We now construct an interesting 3-manifold $B_{3}$ to serve as the base of our F-theory compactifications by performing a sequence of two blow-ups on $\mathbb{P}^{3}$. Let us denote the homogeneous coordinates of our $\mathbb{P}^{3}$ by $w_{1}, w_{2}, w_{3}, w_{4}$ and consider the two $\mathbb{P}^{1}$ 's given by

$$
\mathbb{P}_{1}^{1}: w_{1}=w_{2}=0 \quad \mathbb{P}_{2}^{1}: w_{2}=w_{3}=0
$$

We will blow-up $\mathbb{P}^{3}$ by successively blowing up along $\mathbb{P}_{1}^{1}$ and the proper transform of $\mathbb{P}_{2}^{1}$. In the following, we describe the space $B_{3}^{(0)}$ that results from the first blow-up and our final 3-manifold $B_{3}$ that results from the second blow-up. Both blow-ups are along toric subvarieties of the toric space $\mathbb{P}^{3}$ so we can describe the full geometry in the language of toric geometry. Indeed we will do this but because this 3 -fold is so simple we would like to take some care to describe both its properties and the nature of its toric divisors. All of the toric divisors $D_{i}$ in $B_{3}$ are del Pezzo surfaces or Hirzebruch surfaces and we provide a complete description including a complete enumeration of the curve classes in each $D_{i}$ to which the divisors $D_{j}$ descend. Throughout we will use standard notation for the curve classes of $d P_{n}$ and $\mathbb{F}_{k}$ surfaces. In the case of $d P_{n}$, we will use $h$ for the hyperplane and $e_{i}$ with $i=1, \ldots, n$ for the exceptional curves. For $\mathbb{F}_{k}$ surfaces, we will use $b$ for the base with $b^{2}=-k$ and $f$ for the fiber with $f^{2}=0$ and $f \cdot b=1$.

\section{The first blown-up space $B_{3}^{(0)}$}

The first step is to blow up along the $\mathbb{P}_{1}^{1}$ to obtain the first blown-up space $B_{3}^{(0)}$.

$$
\begin{aligned}
& w_{1}=\tilde{w}_{1} \delta_{1} \\
& w_{2}=\tilde{w}_{2} \delta_{1}
\end{aligned}
$$

where $\delta_{1}$ is the unique holomorphic section of $\mathcal{O}\left(E_{1}\right)$ whose vanishing defines the exceptional divisor $E_{1}$. The resulting 3-manifold is toric and the toric divisors are in the classes $H, H-E_{1}$, and $E_{1}$. We investigate the properties of these toric divisors in turn.

$E_{1}$

As the intersection of two hyperplanes in $\mathbb{P}^{3}$, the normal bundle of $\mathbb{P}_{1}^{1}$ is $\mathcal{O}(1,1)$. This means that the exceptional divisor $E_{1}$ that we get by blowing up along $\mathbb{P}_{1}^{1}$ is $\mathbb{F}_{0}=\mathbb{P}^{1} \times \mathbb{P}^{1}$. What can we say about the restrictions of divisors from $B_{3}^{(0)}$ to $E_{1}$ ? Well we know that $\tilde{w}_{1}=0$ restricts to a copy of the 'base' $b$ of $\mathbb{F}_{0}$ while $w_{3}=0$ restricts to a copy of the fiber. This means that

$$
\begin{aligned}
\left.\mathcal{O}\left(H-E_{1}\right)\right|_{E_{1}} & =\mathcal{O}_{\mathbb{F}_{0}}(b) \\
\left.\mathcal{O}(H)\right|_{E_{1}} & =\mathcal{O}_{\mathbb{F}_{0}}(f)
\end{aligned}
$$

and hence that

$$
\left.\mathcal{O}\left(E_{1}\right)\right|_{E_{1}}=\mathcal{O}_{\mathbb{F}_{0}}(f-b)
$$


$H-E_{1}$

This divisor is the proper transform of a hyperplane that contains the curve $\mathbb{P}_{1}^{1}$ along which we perform the blow-up. Because the curve is of codimension 1 in this hyperplane, this proper transform is again a $\mathbb{P}^{2}$. The restriction of the divisor $H$ is just the line $h$ of this $\mathbb{P}^{1}$ while the exceptional divisor $E_{1}$ also restricts to a line in the class $h$. As a result, we have

$$
\begin{aligned}
\left.\mathcal{O}(H)\right|_{H-E_{1}} & =\mathcal{O}_{\mathbb{P}^{2}}(h) \\
\left.\mathcal{O}\left(E_{1}\right)\right|_{H-E_{1}} & =\mathcal{O}_{\mathbb{P}^{2}}(h)
\end{aligned}
$$

\section{$H$}

This divisor is a hyperplane that does not contain the curve $\mathbb{P}_{1}^{1}$ along which we perform the blow-up. From the perspective of a hyperplane like this, say $w_{4}=0$, our blow-up is just the blow-up of the single point at $w_{1}=w_{2}=0$. As a result, $H$ is a $d P_{1}$ surface. The restriction of $E_{1}$ is the exceptional curve $e_{1}$ of the $d P_{1}$. The restriction of $H-E_{1}$ is the proper transform of a line inside $w_{4}=0$ that contains the point $w_{1}=w_{2}=0$. This is the curve $h-e_{1}$ of the $d P_{1}$. Finally, the restriction of $H$ is clearly the hyperplane $h$ so we have

$$
\begin{aligned}
\left.\mathcal{O}\left(E_{1}\right)\right|_{H} & =\mathcal{O}_{d P_{1}}\left(e_{1}\right) \\
\left.\mathcal{O}\left(H-E_{1}\right)\right|_{H} & =\mathcal{O}_{d P_{1}}\left(h-e_{1}\right) \\
\left.\mathcal{O}(H)\right|_{H} & =\mathcal{O}_{d P_{1}}(h)
\end{aligned}
$$

\section{The second blown-up space $B_{3}$}

Now we proceed to perform the second blow-up. This is along the line $\tilde{w}_{2}=w_{3}=0$ which we denote by $\mathbb{P}_{2}^{1}$

$$
\mathbb{P}_{2}^{1}: \quad \tilde{w}_{2}=w_{3}=0
$$

After this blow-up, the original homogeneous coordinates can be written as

$$
\begin{aligned}
& w_{1}=\tilde{w}_{1} \delta_{1} \\
& w_{2}=\hat{w}_{2} \delta_{1} \delta_{2} \\
& w_{3}=\hat{w}_{3} \delta_{2} \\
& w_{4}=w_{4}
\end{aligned}
$$

where the objects on the right hand side are sections of the indicated bundles

\begin{tabular}{c|c} 
Section & Bundle \\
\hline$\tilde{w}_{1}$ & $\mathcal{O}\left(H-E_{1}\right)$ \\
$\hat{w}_{2}$ & $\mathcal{O}\left(H-E_{1}-E_{2}\right)$ \\
$\hat{w}_{3}$ & $\mathcal{O}\left(H-E_{2}\right)$ \\
$w_{4}$ & $\mathcal{O}(H)$ \\
$\delta_{1}$ & $\mathcal{O}\left(E_{1}\right)$ \\
$\delta_{2}$ & $\mathcal{O}\left(E_{2}\right)$
\end{tabular}


Because the blow-ups are toric we can specify $B_{3}$ by toric data. The matrix of GLSM charges is

$$
\left(\begin{array}{l}
\tilde{w}_{1} \\
\hat{w}_{2} \\
\hat{w}_{3} \\
w_{4} \\
\delta_{1} \\
\delta_{2}
\end{array}\right) \leftrightarrow\left(\begin{array}{ccc}
1 & -1 & 0 \\
1 & -1 & -1 \\
1 & 0 & -1 \\
1 & 0 & 0 \\
0 & 1 & 0 \\
0 & 0 & 1
\end{array}\right) \leftrightarrow\left(\begin{array}{c}
H-E_{1} \\
H-E_{1}-E_{2} \\
H-E_{2} \\
H \\
E_{1} \\
E_{2}
\end{array}\right)
$$

where we also indicate our labels for the divisor classes and our notation for the toric coordinates. The Stanley-Reisner Ideal follows from the blow-up data

$$
S R \sim\left\{\tilde{w}_{1} \hat{w}_{2}, \tilde{w}_{1} \delta_{2}, \hat{w}_{2} \hat{w}_{3}, w_{4} \delta_{1} \delta_{2}, w_{4} \hat{w}_{3} \delta_{1}\right\}
$$

We now describe all of the toric divisors in turn.

\section{$E_{2}$}

The line $\tilde{w}_{2}=w_{3}=0$ is in the class $\left(H-E_{1}\right) \cdot H$ in $B_{3}^{(0)}$. In $B_{3}^{(0)}$, it is in the class $h-e_{1}$ inside the $d P_{1}$ surface $H$ and in the class $h$ in the $\mathbb{P}^{2}$ given by $H-E_{1}$. As a result, its normal bundle in $B_{3}^{(0)}$ is $\mathcal{O}(0,1)$. The exceptional divisor that we get by blowing up $B_{3}^{(0)}$ along this line is therefore an $\mathbb{F}_{1}$ surface. Both $\delta_{1}=0$ and $w_{4}=0$ restrict to copies of the fiber so we have $\left.E_{1}\right|_{E_{2}}=f$ and $\left.H\right|_{E_{2}}=f$. The two sections of the $\mathbb{F}_{1}$ are $\tilde{w}_{2}=0$ and $\tilde{w}_{3}=0$ which are the restrictions of $H-E_{1}-E_{2}$ and $H-E_{2}$. It is easy to verify that $\left(H-E_{1}-E_{2}\right)^{2} E_{2}=-1$ and $\left(H-E_{2}\right)^{2} E_{2}=1$ so that $\left.\left(H-E_{1}-E_{2}\right)\right|_{E_{2}}=b$ and $\left.\left(H-E_{2}\right)\right|_{E_{2}}=f+b$. We can summarize this by saying

$$
\begin{aligned}
\left.\mathcal{O}(H)\right|_{E_{2}} & =\mathcal{O}_{\mathbb{F}_{1}}(f) \\
\left.\mathcal{O}\left(E_{1}\right)\right|_{E_{2}} & =\mathcal{O}_{\mathbb{F}_{1}}(f) \\
\left.\mathcal{O}\left(E_{2}\right)\right|_{E_{2}} & =\mathcal{O}_{\mathbb{F}_{1}}(-b)
\end{aligned}
$$

$E_{1}$

Before the second blow-up, the exceptional divisor $E_{1}$ of $B_{3}^{(0)}$ is a $\mathbb{F}_{0}=\mathbb{P}^{1} \times \mathbb{P}^{1}$. The line $\mathbb{P}_{2}^{1}$ meets $E_{1}$ in exactly one point so after the blow-up $E_{1}$ is the blow-up of $\mathbb{F}_{0}$ at a point, which yields a $d P_{2}$ surface. Let us recall how classes of $\mathbb{F}_{0}$ and the exceptional curve $e$ of such a blow-up are related to the standard parametrization of curves inside $d P_{2}$. Curves in the class $f$ or $b$ that do not pass through the blown-up point map to the class $h-e_{1}$ and $h-e_{2}$ of the $d P_{2}$ surface while the exceptional curve $e$ maps to the line $h-e_{1}-e_{2}$ of the $d P_{2}$

$$
\begin{array}{r}
b \rightarrow h-e_{1} \\
f \rightarrow h-e_{2} \\
e \rightarrow h-e_{1}-e_{2}
\end{array}
$$

Now, the restriction of $H$ and $H-E_{1}$ to $E_{1}$ yield curves in the classes $f$ and $b$ that miss the blown-up point while the restriction of $E_{2}$ yields the exceptional curve $e$. This 
leads to

$$
\begin{aligned}
\left.\mathcal{O}(H)\right|_{E_{1}} & =\mathcal{O}_{d P_{2}}\left(h-e_{2}\right) \\
\left.\mathcal{O}\left(E_{1}\right)\right|_{E_{1}} & =\mathcal{O}_{d P_{2}}\left(e_{1}-e_{2}\right) \\
\left.\mathcal{O}\left(E_{2}\right)\right|_{E_{2}} & =\mathcal{O}_{d P_{2}}\left(h-e_{1}-e_{2}\right)
\end{aligned}
$$

\section{$H$}

Let us now turn to various toric divisors that descend from hyperplanes of the original $\mathbb{P}^{3}$. We start with $H$ which is the class of the divisor $w_{4}=0$. From the perspective of the hyperplane $w_{4}=0$, our first step blows up one point and our second step blows up another one. As a result, $H$ is a $d P_{2}$ surface and the restrictions of the classes $H, E_{1}$, and $E_{2}$ are obvious

$$
\begin{aligned}
\left.\mathcal{O}(H)\right|_{H} & =\mathcal{O}_{d P_{2}}(h) \\
\left.\mathcal{O}\left(E_{1}\right)\right|_{H} & =\mathcal{O}_{d P_{2}}\left(e_{1}\right) \\
\left.\mathcal{O}\left(E_{2}\right)\right|_{H} & =\mathcal{O}_{d P_{2}}\left(e_{2}\right)
\end{aligned}
$$

$H-E_{1}$

Before the second blow-up, $H-E_{1}$ was just a $\mathbb{P}^{2}$. The second blow-up effectively blows up a single point of this $\mathbb{P}^{2}$ resulting in a $d P_{1}$ surface. The restrictions of $H$ and $E_{1}$, which were hyperplanes of the $\mathbb{P}^{2}$ before the second blow-up, are hyperplanes $h$ of the $d P_{1}$ while the restoration of $E_{2}$ is the exceptional line

$$
\begin{aligned}
\left.\mathcal{O}(H)\right|_{H-E_{1}} & =\mathcal{O}_{d P_{1}}(h) \\
\left.\mathcal{O}\left(E_{1}\right)\right|_{H-E_{1}} & =\mathcal{O}_{d P_{1}}(h) \\
\left.\left.\mathcal{O}\left(E_{2}\right)\right|_{H-E_{1}}\right) & =\mathcal{O}_{d P_{1}}(e)
\end{aligned}
$$

$H-E_{2}$

This divisor class is represented by $\hat{w}_{3}=0$ and is the proper transform of the $d P_{1}$ surface $w_{3}=0$ of $B_{3}^{(0)}$ that contains the curve $\mathbb{P}_{2}^{1}$ of the second blow-up. Because $\mathbb{P}_{2}^{1}$ is of codimension 1 inside $w_{3}=0$, this proper transform is again a $d P_{1}$ surface and the restriction of the exceptional line is just a copy of $\mathbb{P}_{2}^{1}$ inside this $d P_{1}$, which is a hyperplane of the class $h$. This means that

$$
\begin{aligned}
\mathcal{O}(H)_{H-E_{2}} & =\mathcal{O}_{d P_{1}}(h) \\
\left.\mathcal{O}\left(E_{1}\right)\right|_{H-E_{2}} & =\mathcal{O}_{d P_{1}}(e) \\
\left.\mathcal{O}\left(E_{2}\right)\right|_{H-E_{2}} & =\mathcal{O}_{d P_{1}}(h)
\end{aligned}
$$

$H-E_{1}-E_{2}$

This divisor class is represented by $\hat{w}_{2}=0$ and is the proper transform of $\mathbb{P}^{2}$ given by $\tilde{w}_{2}=0$ inside $B_{3}^{(0)}$. This $\mathbb{P}^{2}$ contains the curve $\mathbb{P}_{2}^{1}$ so the proper transform is again a $\mathbb{P}^{2}$ 
and all of $H, E_{1}$, and $E_{2}$ restrict to lines

$$
\begin{aligned}
& \left.\mathcal{O}(H)\right|_{H-E_{1}-E_{2}}=\mathcal{O}_{\mathbb{P}^{2}}(h) \\
& \left.\mathcal{O}(H)\right|_{H-E_{1}-E_{2}}=\mathcal{O}_{\mathbb{P}^{2}}(h) \\
& \left.\mathcal{O}(H)\right|_{H-E_{1}-E_{2}}=\mathcal{O}_{\mathbb{P}^{2}}(h)
\end{aligned}
$$

\section{Summary}

We summarize the toric divisors of our final $B_{3}$ and their properties in the following table

\begin{tabular}{cc|ccc|c} 
Divisor Class, $\mathcal{D}$ & Surface Type & $\left.\mathcal{O}(H)\right|_{\mathcal{D}}$ & $\left.\mathcal{O}\left(E_{1}\right)\right|_{\mathcal{D}}$ & $\left.\mathcal{O}\left(E_{2}\right)\right|_{\mathcal{D}}$ & $N_{\mathcal{D} / B_{3}}$ \\
\hline$H$ & $d P_{2}$ & $\mathcal{O}_{d P_{2}}(h)$ & $\mathcal{O}_{d P_{2}}\left(e_{1}\right)$ & $\mathcal{O}_{d P_{2}}\left(e_{2}\right)$ & $\mathcal{O}_{d P_{2}}(h)$ \\
\hline$H-E_{1}$ & $d P_{1}$ & $\mathcal{O}_{d P_{1}}(h)$ & $\mathcal{O}_{d P_{1}}(h)$ & $\mathcal{O}_{d P_{1}}\left(e_{1}\right)$ & $\mathcal{O}_{d P_{1}}$ \\
\hline$H-E_{2}$ & $d P_{1}$ & $\mathcal{O}_{d P_{1}}(h)$ & $\mathcal{O}_{d P_{1}}\left(e_{1}\right)$ & $\mathcal{O}_{d P_{1}}(h)$ & $\mathcal{O}_{d P_{1}}$ \\
\hline$H-E_{1}-E_{2}$ & $\mathbb{P}^{2}$ & $\mathcal{O}_{\mathbb{P}^{2}}(h)$ & $\mathcal{O}_{\mathbb{P}^{2}}(h)$ & $\mathcal{O}_{\mathbb{P}^{2}}(h)$ & $\mathcal{O}_{\mathbb{P}^{2}}(-h)$ \\
\hline$E_{1}$ & $d P_{2}$ & $\mathcal{O}_{d P_{2}}\left(h-e_{2}\right)$ & $\mathcal{O}_{d P_{2}}\left(e_{1}-e_{2}\right)$ & $\mathcal{O}_{d P_{2}}\left(h-e_{1}-e_{2}\right)$ & $\mathcal{O}_{d P_{2}}\left(e_{1}-e_{2}\right)$ \\
\hline$E_{2}$ & $\mathbb{F}_{1}$ & $\mathcal{O}_{\mathbb{F}_{1}}(f)$ & $\mathcal{O}_{\mathbb{F}_{1}}(f)$ & $\mathcal{O}_{\mathbb{F}_{1}}(-b)$ & $\mathcal{O}_{\mathbb{F}_{1}}(-b)$ \\
\hline
\end{tabular}

(B.19)

\section{References}

[1] X. Wen and E. Witten, "World Sheet Instantons and the Peccei-Quinn Symmetry," Phys.Lett. B166 (1986) 397.

[2] M. Dine, N. Seiberg, X. G. Wen, and E. Witten, "Nonperturbative Effects on the String World Sheet," Nucl. Phys. B278 (1986) 769.

[3] M. Dine, N. Seiberg, X. G. Wen, and E. Witten, "Nonperturbative Effects on the String World Sheet. 2," Nucl. Phys. B289 (1987) 319.

[4] S. Kachru, R. Kallosh, A. D. Linde, and S. P. Trivedi, "De Sitter Vacua in String Theory," Phys. Rev. D68 (2003) 046005, 0301240.

[5] V. Balasubramanian, P. Berglund, J. P. Conlon, and F. Quevedo, "Systematics of Moduli Stabilisation in Calabi-Yau Flux Compactifications," JHEP 03 (2005) 007,0502058 .

[6] R. Blumenhagen, M. Cvetič, and T. Weigand, "Spacetime Instanton Corrections in 4D String Vacua - the Seesaw Mechanism for D-Brane Models," Nucl. Phys. B771 (2007) 113-142, 0609191.

[7] L. E. Ibanez and A. M. Uranga, "Neutrino Majorana Masses from String Theory Instanton Effects," JHEP 03 (2007) 052, 0609213.

[8] B. Florea, S. Kachru, J. McGreevy, and N. Saulina, "Stringy Instantons and Quiver Gauge Theories," JHEP 05 (2007) 024, 0610003.

[9] E. Witten, "World-Sheet Corrections via D-Instantons," JHEP 02 (2000) 030, 9907041 . 
[10] E. Witten, "Non-Perturbative Superpotentials in String Theory," Nucl. Phys. B474 (1996) 343-360, 9604030.

[11] R. Donagi and M. Wijnholt, "Model Building with F-Theory," 0802.2969.

[12] C. Beasley, J. J. Heckman, and C. Vafa, "Guts and Exceptional Branes in F-Theory - I," JHEP 01 (2009) 058, 0802.3391.

[13] B. Andreas and G. Curio, "From Local to Global in F-Theory Model Building," J.Geom.Phys. 60 (2010) 1089-1102, 0902.4143.

[14] J. Marsano, N. Saulina, and S. Schäfer-Nameki, "F-Theory Compactifications for Supersymmetric Guts," JHEP 08 (2009) 030, 0904.3932.

[15] A. Collinucci, "New F-Theory Lifts Ii: Permutation Orientifolds and Enhanced Singularities," JHEP 04 (2010) 076, 0906.0003.

[16] R. Blumenhagen, T. W. Grimm, B. Jurke, and T. Weigand, "F-Theory Uplifts and Guts," JHEP 09 (2009) 053, 0906.0013.

[17] J. Marsano, N. Saulina, and S. Schäfer-Nameki, "Monodromies, Fluxes, and Compact Three-Generation F-Theory Guts," JHEP 08 (2009) 046, 0906.4672.

[18] R. Blumenhagen, T. W. Grimm, B. Jurke, and T. Weigand, "Global F-Theory Guts," Nucl. Phys. B829 (2010) 325-369, 0908.1784.

[19] J. Marsano, N. Saulina, and S. Schäfer-Nameki, "Compact F-Theory Guts with $U(1)_{P Q}, " J H E P 04$ (2010) 095, 0912.0272.

[20] T. W. Grimm, S. Krause, and T. Weigand, "F-Theory GUT Vacua on Compact Calabi-Yau Fourfolds," JHEP 07 (2010) 037, 0912.3524.

[21] M. Cvetič, I. García-Etxebarria, and J. Halverson, "Global F-Theory Models: Instantons and Gauge Dynamics," JHEP 01 (2011) 073, 1003.5337.

[22] C.-M. Chen, J. Knapp, M. Kreuzer, and C. Mayrhofer, "Global SO(10) F-Theory Guts," JHEP 10 (2010) 057, 1005.5735.

[23] C.-M. Chen and Y.-C. Chung, "Flipped $S U(5)$ Guts from $E_{8}$ Singularities in F-Theory," JHEP 03 (2011) 049, 1005.5728.

[24] Y.-C. Chung, "On Global Flipped SU(5) Guts in F-Theory," JHEP 03 (2011) $126,1008.2506$.

[25] C.-M. Chen and Y.-C. Chung, "On F-Theory E 6 Guts," JHEP 03 (2011) 129, 1010.5536 .

[26] J. Knapp, M. Kreuzer, C. Mayrhofer, and N.-O. Walliser, "Toric Construction of Global F-Theory Guts," JHEP 03 (2011) 138, 1101.4908.

[27] J. Knapp and M. Kreuzer, "Toric Methods in F-Theory Model Building," Adv.High Energy Phys. 2011 (2011) 513436, 1103.3358.

[28] J. Marsano, H. Clemens, T. Pantev, S. Raby, and H.-H. Tseng, "A Global SU(5) F-Theory Model with Wilson Line Breaking," 1206.6132.

[29] J. Marsano, N. Saulina, and S. Schäfer-Nameki, "A Note on G-Fluxes for F-Theory Model Building," JHEP 11 (2010) 088, 1006.0483. 
[30] J. Marsano, N. Saulina, and S. Schäfer-Nameki, "On G-Flux, M5 Instantons, and $\mathrm{U}(1) \mathrm{S}$ in F-Theory," 1107.1718.

[31] A. P. Braun, A. Collinucci, and R. Valandro, "G-Flux in F-Theory and Algebraic Cycles," Nucl.Phys. B856 (2012) 129-179, 1107.5337.

[32] J. Marsano and S. Schäfer-Nameki, "Yukawas, G-Flux, and Spectral Covers from Resolved Calabi- Yau's," JHEP 11 (2011) 098, 1108.1794.

[33] S. Krause, C. Mayrhofer, and T. Weigand, " $G_{4}$ Flux, Chiral Matter and Singularity Resolution in F-Theory Compactifications," Nucl.Phys. B858 (2012) $1-47,1109.3454$.

[34] T. W. Grimm and H. Hayashi, "F-Theory Fluxes, Chirality and Chern-Simons Theories," 1111.1232 .

[35] A. P. Braun, A. Collinucci, and R. Valandro, "Algebraic Description of G-Flux in F-Theory: New Techniques for F-Theory Phenomenology," 1202.5029.

[36] M. Kuntzler and S. Schäfer-Nameki, "G-Flux and Spectral Divisors," 1205.5688.

[37] S. Krause, C. Mayrhofer, and T. Weigand, "Gauge Fluxes in F-Theory and Type IIB Orientifolds," JHEP 1208 (2012) 119, 1202.3138.

[38] A. Collinucci and R. Savelli, "On Flux Quantization in F-Theory," JHEP 1202 (2012) 015, 1011.6388.

[39] A. Collinucci and R. Savelli, "On Flux Quantization in F-Theory Ii: Unitary and Symplectic Gauge Groups," JHEP 1208 (2012) 094, 1203.4542.

[40] T. W. Grimm and T. Weigand, "On Abelian Gauge Symmetries and Proton Decay in Global F- Theory Guts," Phys. Rev. D82 (2010) 086009, 1006.0226.

[41] M. J. Dolan, J. Marsano, N. Saulina, and S. Schäfer-Nameki, "F-Theory Guts with U(1) Symmetries: Generalities and Survey," Phys.Rev. D84 (2011) 066008, 1102.0290 .

[42] T. W. Grimm, M. Kerstan, E. Palti, and T. Weigand, "Massive Abelian Gauge Symmetries and Fluxes in F-Theory," JHEP 1112 (2011) 004, 1107.3842.

[43] D. R. Morrison and D. S. Park, "F-Theory and the Mordell-Weil Group of Elliptically-Fibered Calabi-Yau Threefolds," 1208.2695.

[44] R. Donagi, A. Grassi, and E. Witten, "A Nonperturbative Superpotential with $E_{8}$ Symmetry," Mod.Phys.Lett. A11 (1996) 2199-2212, 9607091.

[45] S. H. Katz and C. Vafa, "Geometric Engineering of $\mathcal{N}=1$ Quantum Field Theories," Nucl.Phys. B497 (1997) 196-204, 9611090.

[46] D.-E. Diaconescu and S. Gukov, "Three-Dimensional $\mathcal{N}=2$ Gauge Theories and Degenerations of Calabi-Yau Four Folds," Nucl.Phys. B535 (1998) 171-196, 9804059 .

[47] O. J. Ganor, "A Note on Zeros of Superpotentials in F Theory," Nucl.Phys. B499 (1997) 55-66, 9612077.

[48] R. Blumenhagen, A. Collinucci, and B. Jurke, "On Instanton Effects in F-Theory," JHEP 08 (2010) 079, 1002.1894. 
[49] R. Donagi and M. Wijnholt, "MSW Instantons," 1005.5391.

[50] J. M. Maldacena, A. Strominger, and E. Witten, "Black Hole Entropy in M Theory," JHEP 9712 (1997) 002, 9711053.

[51] T. W. Grimm, M. Kerstan, E. Palti, and T. Weigand, "On Fluxed Instantons and Moduli Stabilisation in IIB Orientifolds and F-Theory," 1105.3193.

[52] R. Blumenhagen, S. Moster, and E. Plauschinn, "Moduli Stabilisation Versus Chirality for MSSM Like Type IIB Orientifolds," JHEP 01 (2008) 058, 0711.3389 .

[53] M. Cvetič, I. Garcia Etxebarria, and J. Halverson, "Three Looks at Instantons in F-Theory - New Insights from Anomaly Inflow, String Junctions and Heterotic Duality," JHEP 11 (2011) 101, 1107.2388.

[54] M. Bianchi, A. Collinucci, and L. Martucci, "Magnetized E3-Brane Instantons in F-Theory," JHEP 1112 (2011) 045, 1107.3732.

[55] M. Kerstan and T. Weigand, "Fluxed M5-Instantons in F-Theory," 1205.4720.

[56] C. Vafa, "Evidence for F-Theory," Nucl. Phys. B469 (1996) 403-418, 9602022.

[57] D. Baumann, A. Dymarsky, I. R. Klebanov, J. M. Maldacena, L. P. McAllister, et al., "On D3-Brane Potentials in Compactifications with Fluxes and Wrapped D-Branes," JHEP 0611 (2006) 031, 0607050.

[58] R. Blumenhagen, M. Cvetič, D. L "ust, R. Richter, and T. Weigand, "Non-Perturbative Yukawa Couplings from String Instantons," Phys. Rev. Lett. 100 (2008) 061602, 0707.1871.

[59] E. I. Buchbinder, R. Donagi, and B. A. Ovrut, "Superpotentials for vector bundle moduli," Nucl.Phys. B653 (2003) 400-420, 0205190.

[60] M. Esole and S.-T. Yau, "Small Resolutions of SU(5)-models in F-Theory," 1107.0733 .

[61] J. J. Heckman, A. Tavanfar, and C. Vafa, "The Point of $E_{8}$ in F-theory GUTs," JHEP 08 (2010) 040, 0906.0581.

[62] G. Curio, "World-Sheet Instanton Superpotentials in Heterotic String Theory and Their Moduli Dependence," JHEP 09 (2009) 125, 0810.3087.

[63] G. Curio, "Perspectives on Pfaffians of Heterotic World-sheet Instantons," JHEP 0909 (2009) 131, 0904.2738.

[64] G. Curio, "On the Heterotic World-Sheet Instanton Superpotential and Its Individual Contributions," JHEP 08 (2010) 092, 1006.5568.

[65] M. Kreuzer and H. Skarke, "Classification of Reflexive Polyhedra in Three-Dimensions," Adv.Theor.Math.Phys. 2 (1998) 847-864, 9805190.

[66] E. Witten, "Five-Brane Effective Action in M Theory," J.Geom.Phys. 22 (1997) 103-133, 9610234.

[67] C. Beasley and E. Witten, "New Instanton Effects in String Theory," JHEP 02 (2006) 060, 0512039. 
[68] E. Buchbinder, R. Donagi, and B. A. Ovrut, "Vector Bundle Moduli and Small Instanton Transitions," JHEP 06 (2002) 054, 0202084.

[69] E. I. Buchbinder, R. Donagi, and B. A. Ovrut, "Vector Bundle Moduli Superpotentials in Heterotic Superstrings and M-theory," JHEP 07 (2002) 066, 0206203 .

[70] E. Witten, "On Flux Quantization in M-theory and the Effective Action," $J$. Geom. Phys. 22 (1997) 1-13, 9609122.

[71] R. Donagi and M. Wijnholt, "Higgs Bundles and UV Completion in F-Theory," 0904.1218.

[72] N. Saulina, "Topological Constraints on Stabilized Flux Vacua," Nucl.Phys. B720 (2005) 203-210, 0503125.

[73] R. Kallosh, A.-K. Kashani-Poor, and A. Tomasiello, "Counting Fermionic Zero Modes on M5 with Fluxes," JHEP 0506 (2005) 069, 0503138.

[74] D. L "ust, S. Reffert, W. Schulgin, and P. K. Tripathy, "Fermion Zero Modes in the Presence of Fluxes and a Non-Perturbative Superpotential," JHEP 0608 (2006) 071, 0509082.

[75] D. Tsimpis, "Fivebrane Instantons and Calabi-Yau Fourfolds with Flux," JHEP 0703 (2007) 099, 0701287.

[76] M. Kreuzer and H. Skarke, "Complete Classification of Reflexive Polyhedra in Four Dimensions," Adv. Theor. Math. Phys. 4 (2002) 1209-1230, 0002240. 